\title{
Rehabilitation of structures below the earths surface using fibre reinforced polymer shell augers and fabrication of plastic compounder
}

\author{
Kumar Venkatesh Karri
}

Follow this and additional works at: https://researchrepository.wvu.edu/etd

\section{Recommended Citation}

Karri, Kumar Venkatesh, "Rehabilitation of structures below the earths surface using fibre reinforced polymer shell augers and fabrication of plastic compounder" (2016). Graduate Theses, Dissertations, and Problem Reports. 4003.

https://researchrepository.wvu.edu/etd/4003

This Problem/Project Report is protected by copyright and/or related rights. It has been brought to you by the The Research Repository @WVU with permission from the rights-holder(s). You are free to use this Problem/Project Report in any way that is permitted by the copyright and related rights legislation that applies to your use. For other uses you must obtain permission from the rights-holder(s) directly, unless additional rights are indicated by a Creative Commons license in the record and/ or on the work itself. This Problem/Project Report has been accepted for inclusion in WVU Graduate Theses, Dissertations, and Problem Reports collection by an authorized administrator of The Research Repository @ WVU. For more information, please contact researchrepository@mail.wvu.edu. 


\title{
REHABILITATION OF STRUCTURES BELOW THE EARTHS SURFACE USING FIBRE REINFORCED POLYMER SHELL AUGERS AND FABRICATION OF PLASTIC COMPOUNDER
}

\author{
Kumar Venkatesh Karri \\ Problem report submitted to the \\ Benjamin M. Statler College of Engineering and Mineral Resource \\ At West Virginia University \\ In partial fulfillment of the requirements \\ For the degree of
}

\section{Master of Science \\ In \\ Civil Engineering}

\author{
Approved by \\ Hota V. S. GangaRao, Ph.D., P.E., Chair \\ Ruifeng Liang, Ph.D. \\ Mark L.Skidmore, M.S., P.E.
}

\section{Department of Civil and Environmental Engineering}

\author{
Morgantown, West Virginia
}

2016

Keywords: Underwater rehabilitation, GFRP shell, augering, underwater wrapping, Aquawrap

Copyright 2016 Kumar Venkatesh Karri 


\title{
Abstract \\ REHABILITATION OF STRUCTURES EXTENDING THE EARTHS SURFACE USING FIBRE REINFORCED POLYMER SHELL AUGERS AND FABRICATION OF PLASTIC COMPOUNDER
}

\author{
Kumar Venkatesh Karri \\ Constructed Facilities Centre, West Virginia University
}

Many ageing highway bridges are in distress, requiring urgent repairs or rehabilitation. About $10 \%$ of the total highway bridges require rehabilitation in United States of America (Houlihan, April 2015). Reconstruction of these bridges requires large sums of money and time consuming conventional rehab schemes. Herein, a novel rehab scheme with Fiber Reinforced Polymer (FRP) composites has been evaluated in the laboratory as FRPs exhibit high strength to weight ratio, high stiffness and excellent corrosion resistance.

Current methods to repair pile systems are limited by access issues as the piles are extended typically underwater and below the mudline. Traditional methods require cofferdams or other barrier systems to work in the dry or divers to work in the wet. Access is also hindered as the piles support the existing bridge; i.e. there is a structure overhead and repairs have to be made around the existing pile supporting super structure. Proper repairs typically require digging below the mudline, which complicates any traditional repair method. The repair method proposed herein seeks to solve both the access and excavation issues by combining an augering attachment to FRP formwork that can be installed around an existing pile. Twisting the FRP formwork engages the auger, which drives the forms below the mudline. This method can be adopted above water, eliminating the need for barrier systems or divers. The auger attachment can be modified based on soil conditions. As the shell bores down, additional shells can be attached to the previously attached shell by an overlapping joint and also reinforcing the shell with FRP composite wrap to prevent potential buckling under torsion and to minimize moisture ingress through the shell. Based on the compactness of the soil and/or hardness, FRP composites can be selected with proper fiber volume fraction and orientation.

In this study, field conditions have been replicated in lab and three different kinds of Auger attachments to the FRP composite shell are tested with manual application of torque to understand various challenges in driving these shells below the mudline. The stresses developed in the shell, both by the application of torque and resistance offered by soil are measured using of strain gages. The strain gage readings are evaluated with respect to strain limits of FRP composite to understand the FRP-soil interaction and to attain safe shell design.

Plastic waste is the other major issue at present. The production is increasing every year rapidly but the recycling is not. The plastic waste that is not recycled is dumped in oceans or landfilled causing disturbance in ecological cycle. To reduce ecological disturbance, a 
compounding machine converting the plastic waste into a structurally useful product has been discussed herein as a second part of this report. 


\section{Acknowledgement}

First and foremost, I would like to thank my advisor Dr. Hota GangaRao for giving me an opportunity to work under him and to pursue a Master of Science in Civil Engineering. His continuous guidance, support had a very positive influence on me throughout my graduate student life. Apart from his vast knowledge and expertise, his spiritual mind makes him unique advisor and mentor.

I would also thank Mark Skidmore for his patience and valuable inputs towards my work agreeing to serve on my graduate advisory committee. I also thank Dr. Ruifeng Liang for serving on my graduate advisory committee. And a special thanks to Jerry Nestor for helping me throughout the lab testing without whom my work wouldn't have been possible.

Last but not least, I would like to thank my parents Suvarchla Karri \& Vasudevarao Karri, uncle Dr. Prasad Rao Mallela, my sisters Niroopa Karri \& Bhavana Naredla and ally my friends for their unconditional love and affection towards me. Without their support and motivation, I wouldn't have been any better person than today. 


\section{Table of Contents}

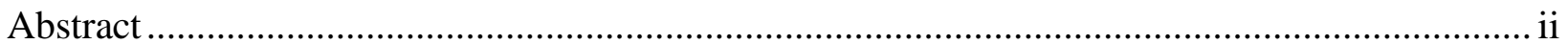

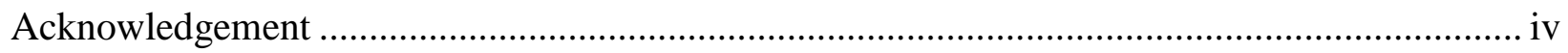

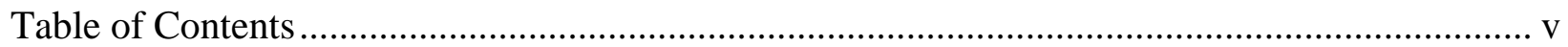

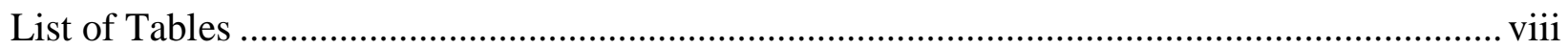

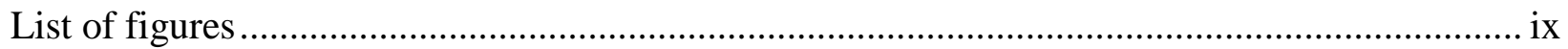

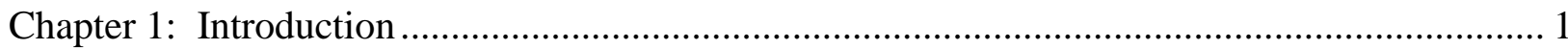

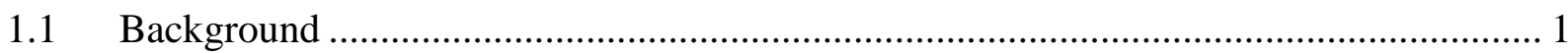

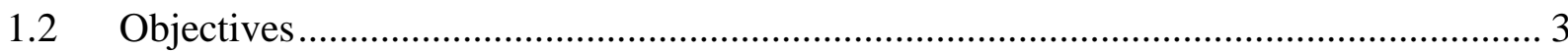

1.3 Scope and organization of the Problem report .............................................................. 4

Chapter 2. Literature Review........................................................................................ 5

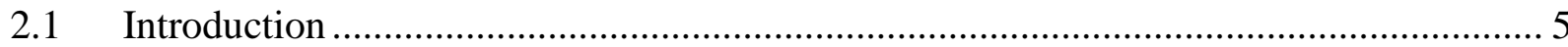

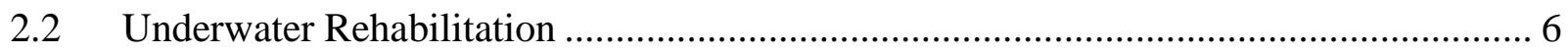

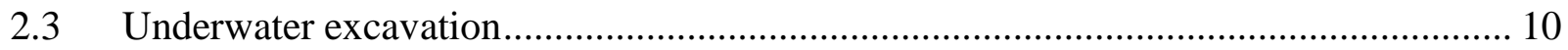

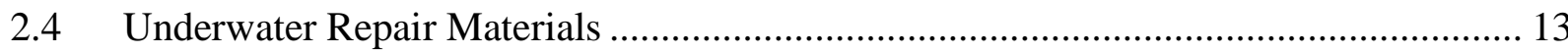

2.4.1 Types of wraps and resins ................................................................................ 14

2.4.2 Fiber Reinforced Polymer Shell/Jacket ............................................................... 18

Chapter 3. Fiber Reinforced Polymer Shell-Auger System …………………….................... 20

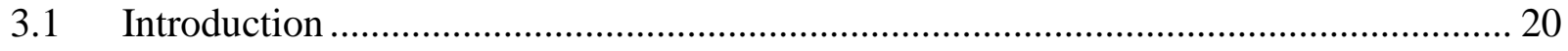


Fiber Reinforced Polymer shell.................................................................... 21

3.3 Design of Auger Attachment............................................................................... 23

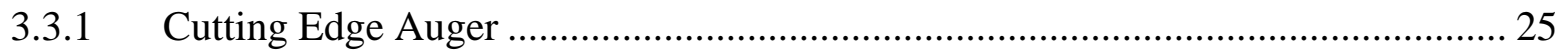

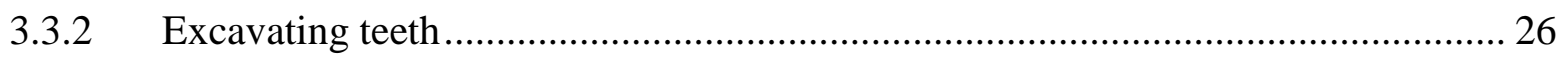

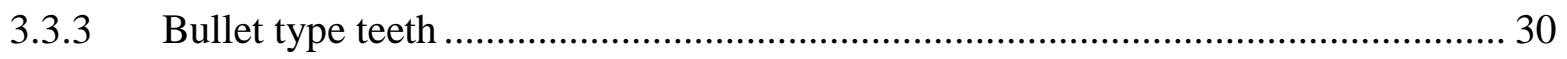

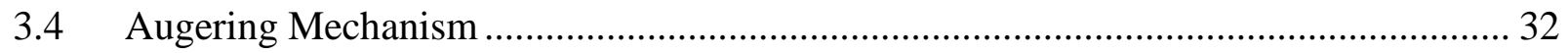

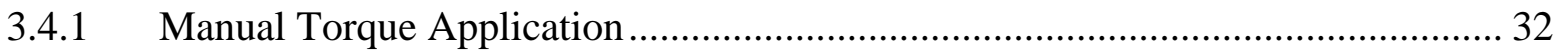

3.4.2 Mechanical Torque Application ............................................................... 34

3.4.3 Vertical Load/Vibration ........................................................................... 35

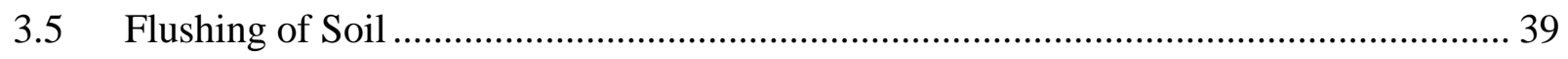

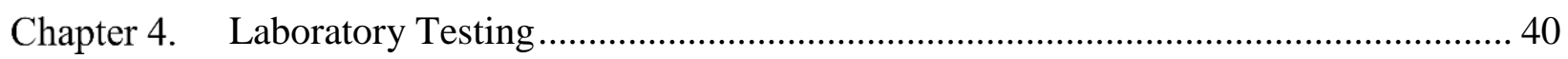

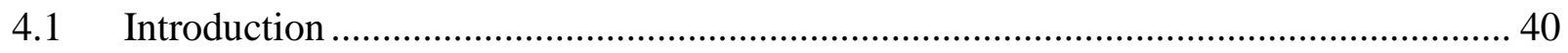

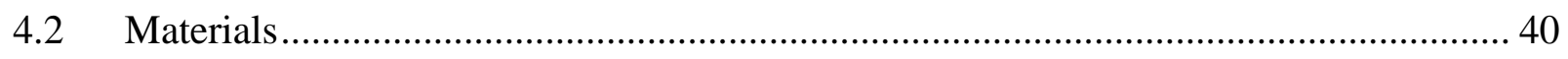

4.2.1 Fiber Reinforced Polymer Shell/Jacket .................................................... 40

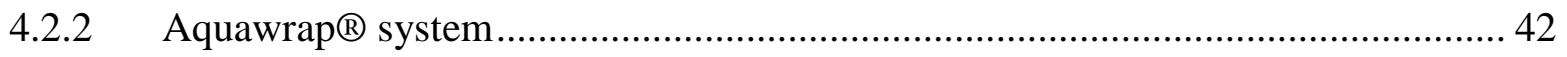

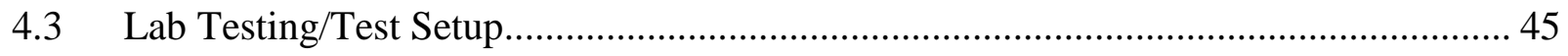

4.3.1 Positioning of Jacket and Auger around the Steel Column ............................... 46

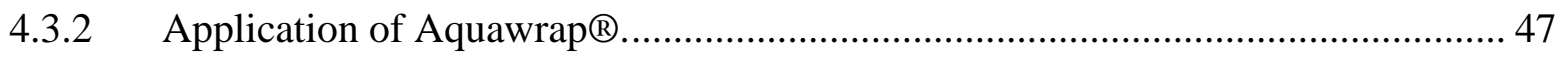

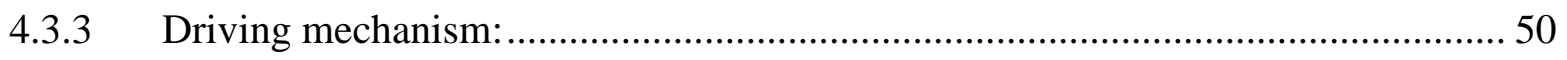

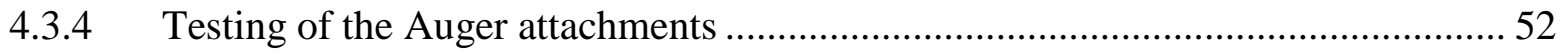


4.3.5 Comparison of Auger attachments............................................................ 54

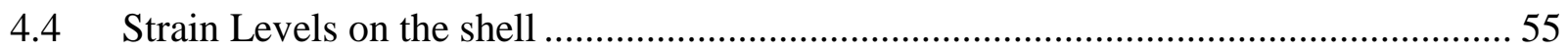

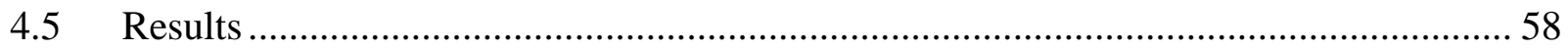

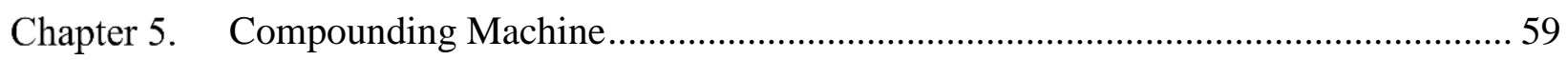

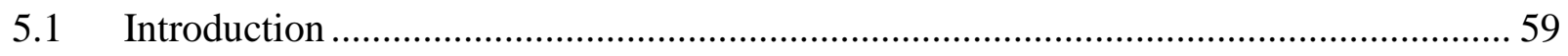

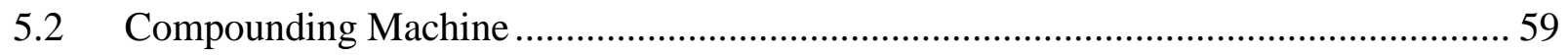

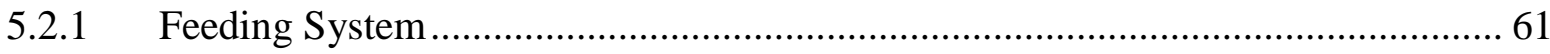

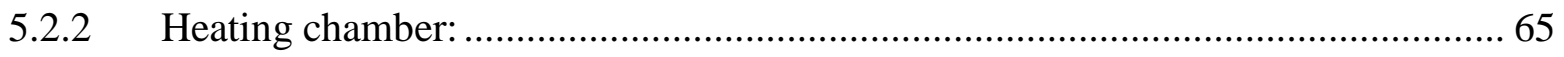

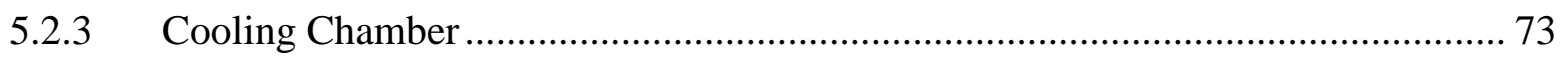

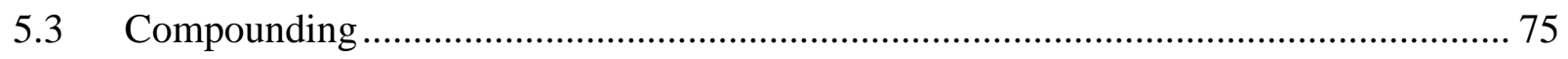

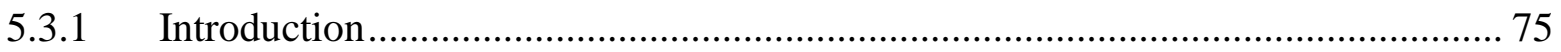

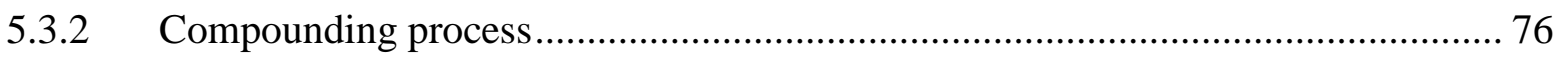

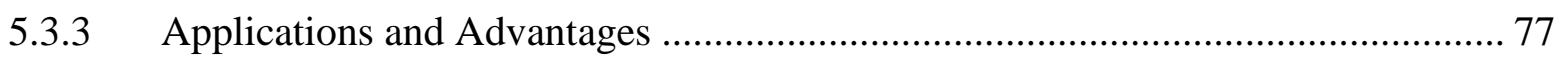

Chapter 6. Conclusions and Recommendations ....................................................... 78

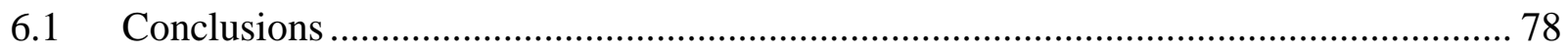

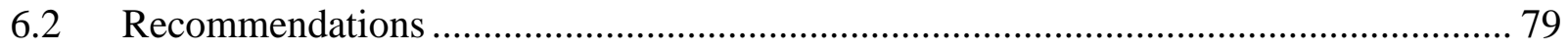

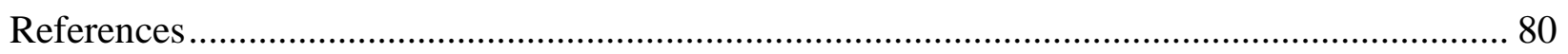

Appendix A- Stress Calculation of the Shell Wrap composite ............................................. 83

Appendix B - Maximum Vertical load and Torque applicable on shell ................................. 85 


\section{List of Tables}

Table 4. 1: Technical properties of FX-70 (FX-70 Structural Repair and Protective system, 2016) ............ 40

Table 4. 2: Properties of Aqua wrap (Highly Conformable Tape and Woven Roving Fabric Constructions,

2015)

Table 4. 3: Technical specifications of Aquawrap Composite (Highly Conformable Tape and Woven

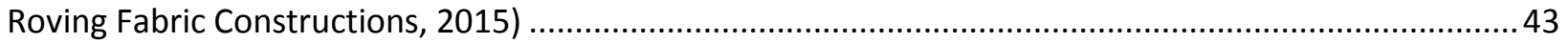

Table 4. 4: Properties of BP-4 Primer /Adhesive (BP-4 Primer/Adhesive/Coating, 2012)........................ 43 


\section{List of figures}

Figure 2-1: Illustration of steel coffer dam (Michael J. Garlich, John E. O’Leary, Katherine C.

Heringhaus, \& Thomas J. Collins, April 2010)........................................................................ 7

Figure 2-2: Water filled bladder (Dam-It-Dams, 2016) ......................................................... 7

Figure 2-3: Diver, with support crew (Soti, 2014) ……...................................................... 9

Figure 2-4: Video monitoring and communication setup (Soti, 2014)....................................... 9

Figure 2-5: Equipment on Floating Platform (Michael J. Garlich, John E. O’Leary, Katherine C.

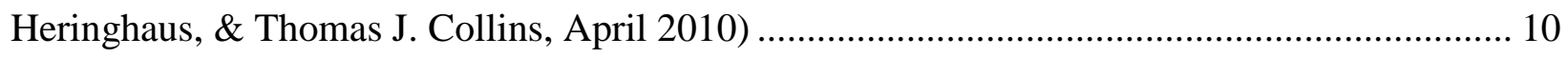

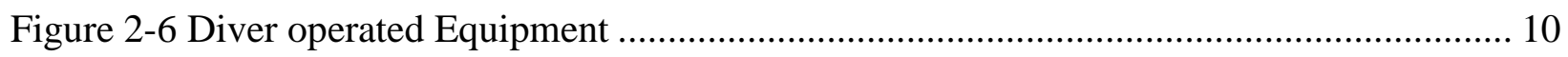

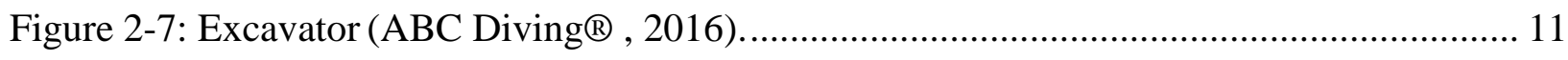

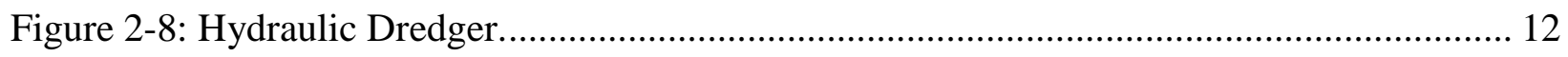

Figure 2-9: The suction pump with the agitators (ABC Diving $®$, 2016)................................... 12

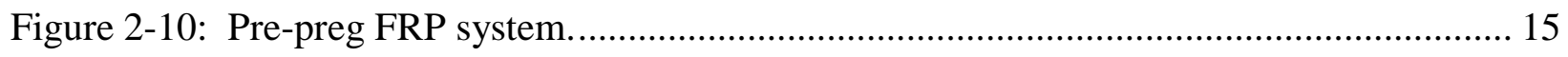

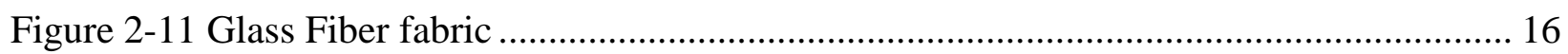

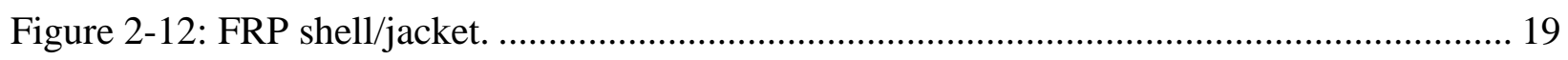

Figure 2-13 : Tongue and Groove Joint (Simpsons Strong-Ti, 2016) ......................................... 19

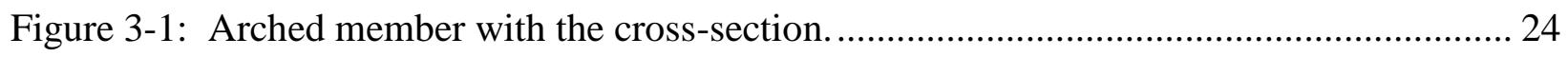

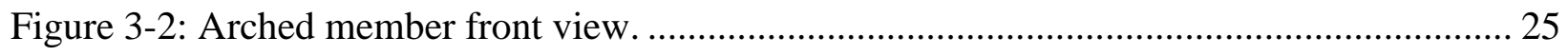

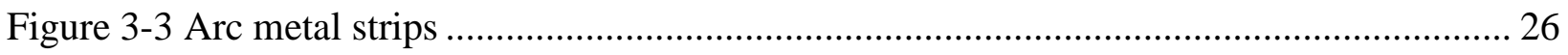

Figure 3-4: Additional Helical attachments to Auger................................................................... 27

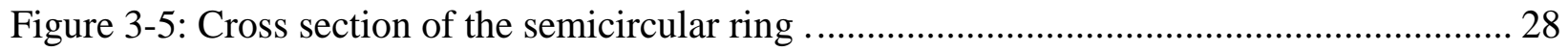

Figure 3-6: Rectangular block from which the cutting teeth is made........................................... 28 
Figure 3-7: Cutting teeth (bottom side).

Figure 3-8: Cutting teeth with the cutting edge. ............................................................... 29

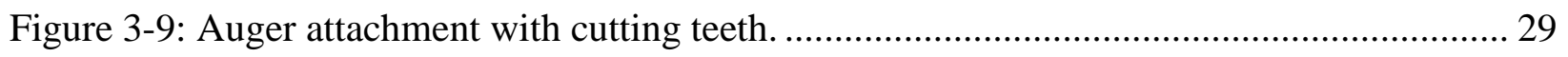

Figure 3-10: Conical bullet teeth and block holder. .......................................................... 30

Figure 3-11: Bullet teeth with the blocks attached to the circular ring................................... 31

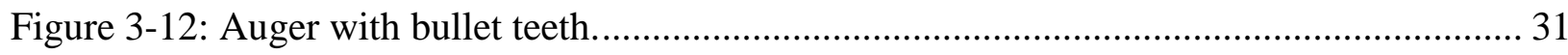

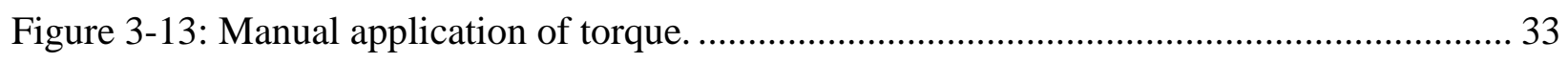

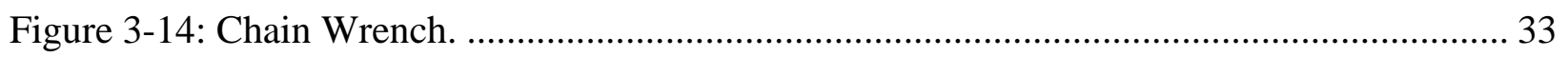

Figure 3-15: Sand bags Used to apply Vertical loads...................................................... 35

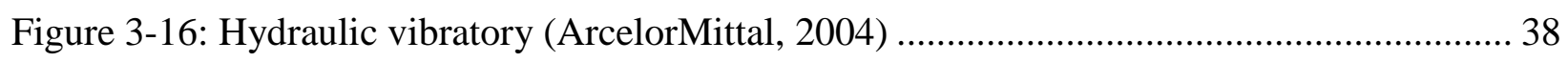

Figure 3-17: A free hanging vibrator (left) and leader-mounted vibrator (right) (ArcelorMittal,

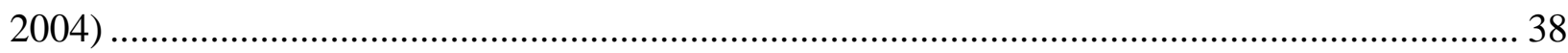

Figure 3-18: Principle of operation (Kenneth Viking, May 2002) ........................................ 39

Figure 4-1: Simpsons Strong-TieFx-70 jacket............................................................ 41

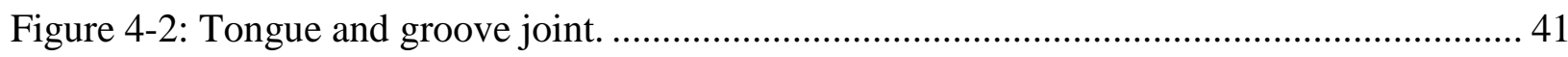

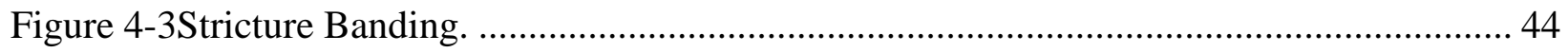

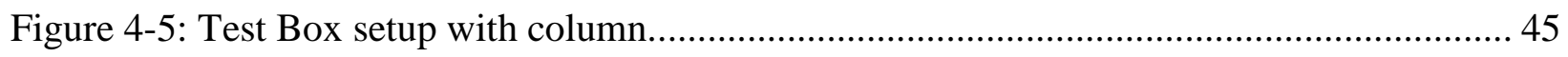

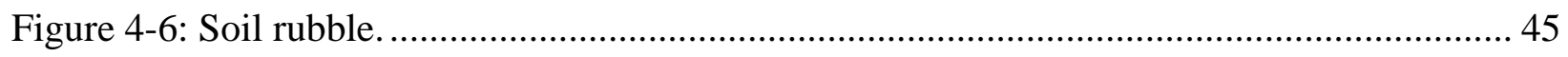

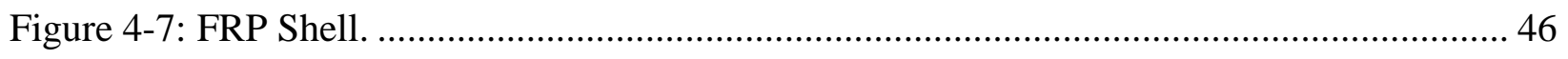

Figure 4-8: Auger adjoined to the FRP shell with bolts and Epoxy. ..................................... 47

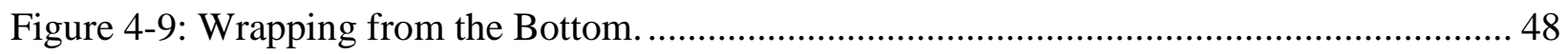

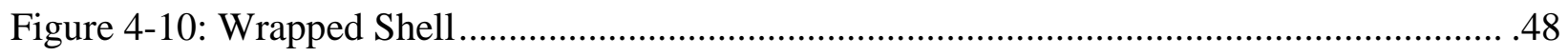

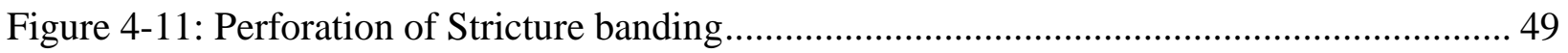




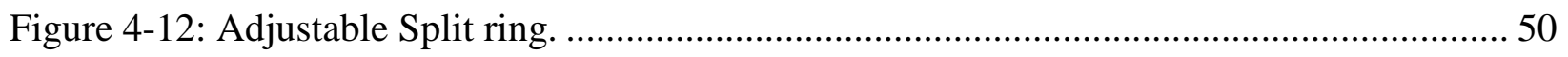

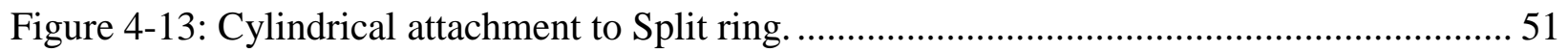

Figure 4-14: Hollow cylindrical tube to produce torque. ………………………………........... 51

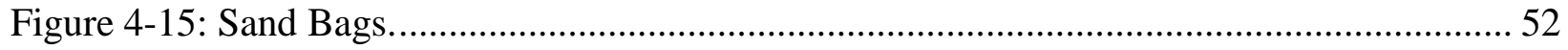

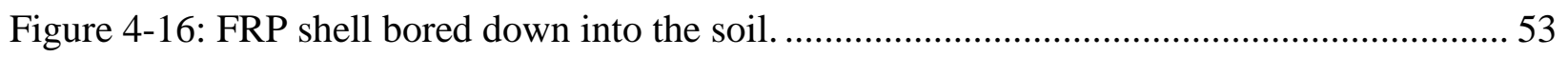

Figure 4-17: The FRP shell going down as soil is pushed aside. ............................................. 53

Figure 4-20: Figure Strain Vs Time Graph of all the four Strain gages. ...................................... 56

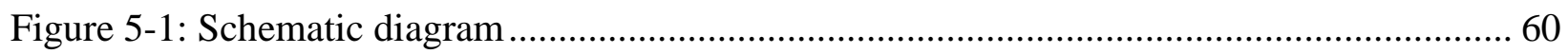

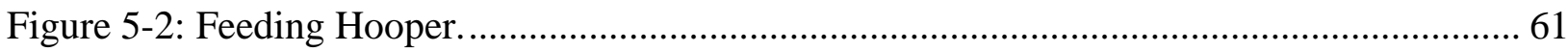

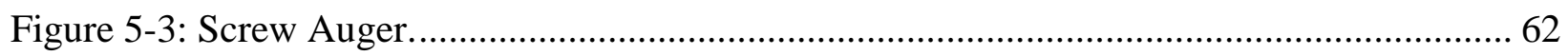

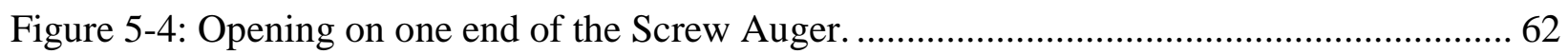

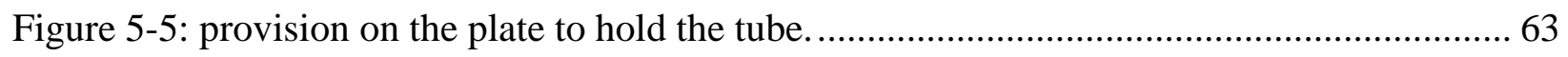

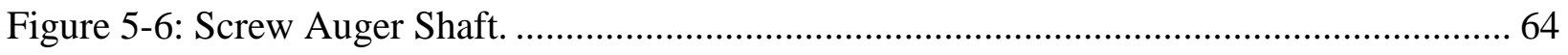

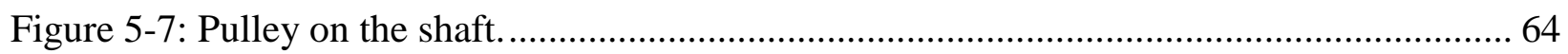

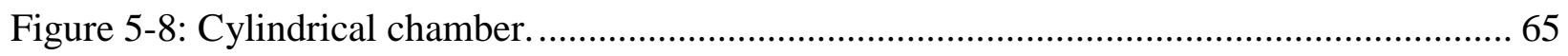

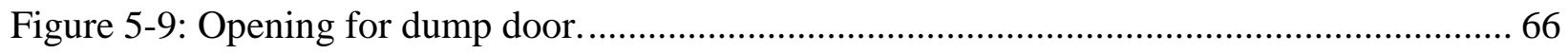

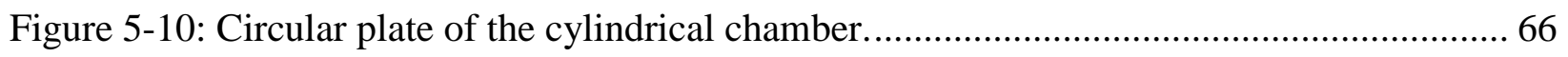

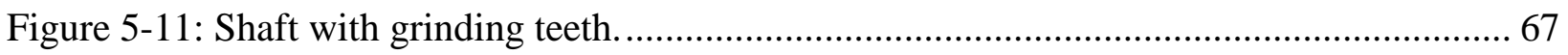

Figure 5-12: Semi Circular components bolted and welded together to form cylindrical chamber.

Figure 5-13: parallel plates on which the chamber is mounted................................................. 68

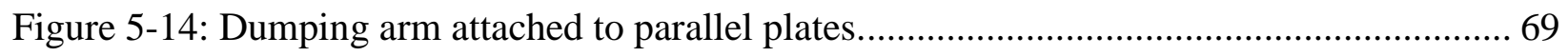

Figure 5-15: Parallel plates with provision to hold the heating chamber. .................................... 69 
Figure 5-16: Portion of shaft that remains inside the heating chamber.

Figure 5-17: Grinding blocks mounted on shaft. ....................................................... 71

Figure 5-18: Slide in and bolt provision for the blocks................................................ 71

Figure 5-19: Both the bearing placed on either sides of the shaft. ......................................... 72

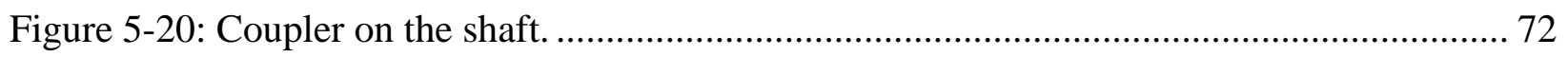

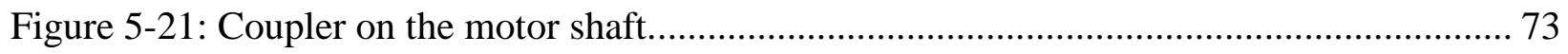

Figure 5-22: the hollow shell with cooling chamber. ...................................................... 74

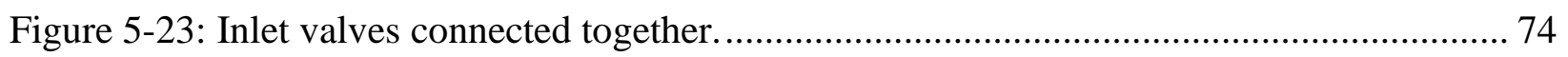

Figure 5-24: Flow Chart of the Compounding Process ..................................................... 76 


\section{Chapter 1: Introduction}

\subsection{Background}

Structures submerged partially in water are prone to rapid deterioration and rate of deterioration becomes higher at water splash zones. Freezing and thawing effects, sulphate attack and corrosion of structures especially at the splash zones result in higher rates deterioration. The deterioration rate is related to the exposure of a structural member to harsh chemicals, freeze-thaw cycles and varying thermo-mechanical conditions, including ever increasing live loads and ever decreasing material stiffness.

With increasing number of underwater structures requiring rehabilitation and ageing of highway bridges, there is need to have better understanding of existing and new technologies to come up with intelligent schemes of rehabilitation that are both cost effective and time efficient.

Typical underwater rehabilitation of structures including foundation is done "in-the-dry" or "in-the-wet" environment (Michael J. Garlich, John E. O’Leary, Katherine C. Heringhaus, \& Thomas J. Collins, April 2010). "In-the-dry" refers to a barrier preventing water flow during the rehab work such as cofferdams, constructing dikes or portable dams. Working "in-the-dry' is the most commonly used method as there is direct access to inspect and rehabilitate a structure. However, creating dry conditions using barriers results in increased cost to rehabilitation. "In-thewet" repairs utilize diver driven schemes, remotely operated tools or underwater tools. The use of equipment for underwater repair saves the time for site preparation compared to a dry site preparation. However, underwater repairs generally are slower to complete, require highly skilled workers and can be unfeasible based on the water conditions (i.e. strong currents). If the substructure lying below the mudline needs to be rehabilitated, digging below the mudline is 
required. Finally if the repair were to happen with the existing pile in place and a functional superstructure then standard methods of foundation installation will be more difficult to adopt, if not impossible.

Thus, there is a need to come up with new technologies that permit repairs to be completed on existing structures while keeping the work crew above water; thus minimizing the use of costly equipment and in-situ form work. The integration of underwater repair with augering techniques makes the rehabilitation process cost effective and time efficient without disturbing the integrity of the surrounding soil foundations or intruding into user convenience.

The other major issue in the present world is to find ways to recycle plastic waste. Plastics are non-biodegradable materials, disintegrating only after few thousands of years. The production of plastics is increasing by about 10 million metric tons a year (Statista, 2016). About 8 million metric tons of plastic waste finds in the ocean (Lytle, 2016), which is highly toxic to our ecosystem. It not only affects the marine life, but also affects human health as toxic wastes enter into the food chain.

Compounding is one method through which the plastics can be not only recycled but also combined with fibers to create greater structural capabilities and arrive at value added end products. Better understanding of the fabrication and working of machines that compound the constituent materials is required to ensure smooth compounding process and minimizing landfill of used plastics. Herein, such compounding machine development has been detailed after fabricating it in the Major Units Laboratory along with the help of local fabricator. 


\subsection{Objectives}

The objectives of this study are:

- To design a fiber reinforced polymer shell system with an auger attachment for underwater structures rehabilitation in a non-intrusive way.

- To come up with a suitable driving mechanism for the fiber reinforced polymer shell system with auger attachment.

- To check the induced stresses (circumferential \& longitudinal) in the shell during the augering process and establish ease of augering under different soil conditions, etc.

- To fabricate a compounding machine that would compound comingled/mixed plastics, reducing the cleaning and separating works involved in other plastic recycling process. 


\subsection{Scope and organization of the Problem report}

Chapter 2 provides a comprehensive literature review about various methods for rehabilitation of underwater structures which are currently used in practice, advantages and disadvantages of existing methods and various augering techniques to bore below the mudline and various underwater repair materials made of fiber reinforced polymer composites.

Chapter 3 discusses in detail the proposed repair system for rehabilitation of structures below mudline which is novel and safe for onsite working crew. It also gives an overview of how the FRP shell and auger attachment function in addition to describing the design of three auger attachments. It also discusses the various augering mechanisms for application of torque and downward thrust.

Chapter 4 includes the test setup for the fiber reinforced polymer shells with various auger attachments, joining of the auger attachment to the shell and process of wrapping around the shell, including procedures for the driving mechanisms of the auger.

Chapter 5 includes detailed explanations of various components of a rotary compounding machine and its fabrication. It also discusses the process of compounding to turn plastic waste into a value added product. 


\section{Chapter 2. Literature Review}

\subsection{Introduction}

One in ten United States bridges are in urgent need of repair (Nawaguna, April 2014), with around 250 million crossings happen every day on these bridges (Houlihan, April 2015). Corrosion of piles in splash zones is one of the major causes of bridge deterioration.

Corrosion of steel piles and reinforcement is the major cause of overall deterioration in marine environments. The deterioration includes loss of cross-sectional steel resulting in loss of strength and durability of structure effecting its serviceability. Many studies have been conducted to have a better understanding of underwater repairs and the materials that would yield better results for underwater rehabilitation.

Most of the existing underwater repair methods include the use of support structures to maintain a dry work site or divers and underwater repair equipment. If a substructure below the mudline needs rehabilitation, excavation in combination with above-mentioned methods is necessary. Developing dry environment for underwater structural systems requires timeconsuming site preparation. In addition, diving operations are hazardous, slow to complete and require highly trained personal.

Traditional repair methods include attaching additional steel to replace the corroded steel and the application of protective paint layers or patching and FRP jacketing or reinforced concrete members. Fiber reinforced polymer wraps/shell not only protect the structure from corrosion but can also enhance the structural capacity. Though the fiber reinforced polymer composites material 
cost is higher, on the whole the total rehabilitation cost of a project is lower and cost effective in terms of life cycle costs.

This chapter mainly focuses on the review of existing methods of underwater rehabilitation and excavation techniques. A discussion on FRP materials is also included.

\subsection{Underwater Rehabilitation}

The underwater rehabilitation can be classified into rehabilitation "in-the-dry" and "in-thewet" as discussed in "Underwater Bridge Repair, Rehabilitation, and Countermeasures" (Michael J. Garlich, John E. O’Leary, Katherine C. Heringhaus, \& Thomas J. Collins, April 2010) a manual published by the Federal Highway Administration, U.S. Department of Transportation to summarize how underwater repairs may best be completed. The manual not only discusses the existing underwater repair techniques and environmental considerations but also the materials and equipment that can be used for rehabilitation.

Repairs in-the-dry involve constructing a barrier between the repair site and water. Once the barrier is constructed, the repair site is dewatered to carry on the inspection and repair work. The most common methods in practice to obtain dry working conditions for field repairs are cofferdams, dikes and barrier systems. The type of barrier depends on various factors such as the river flow, water level, and soil profile and work duration (Michael J. Garlich, John E. O’Leary, Katherine C. Heringhaus, \& Thomas J. Collins, April 2010).

Cofferdams (Figure 2-1) are temporary barriers commonly made of wood, steel or polymer sheet piling. Cofferdams are good for higher water levels and strong river currents to counteract soil and water pressures (ABC Diving Ltd, 2016). Dikes are barriers that can be made out of 
inexpensive materials like plastic sheathing, sand bags and compacted clays (Michael J. Garlich, John E. O’Leary, Katherine C. Heringhaus, \& Thomas J. Collins, April 2010). Dikes are good for shallow depths and can be constructed and placed around a repair site. Water filled bladders (Figure 2-2) can also be used as barriers for shallow waters.

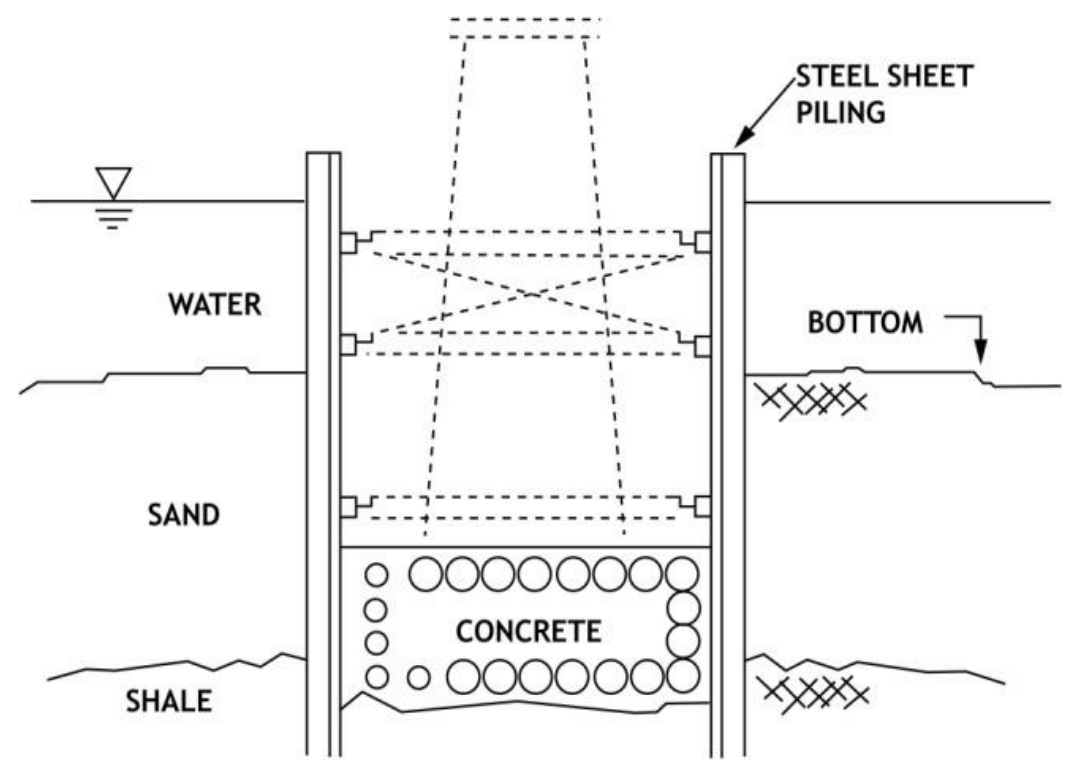

Figure 2-1: Illustration of steel coffer dam (Michael J. Garlich, John E. O’Leary, Katherine C. Heringhaus, \& Thomas J. Collins, April 2010).

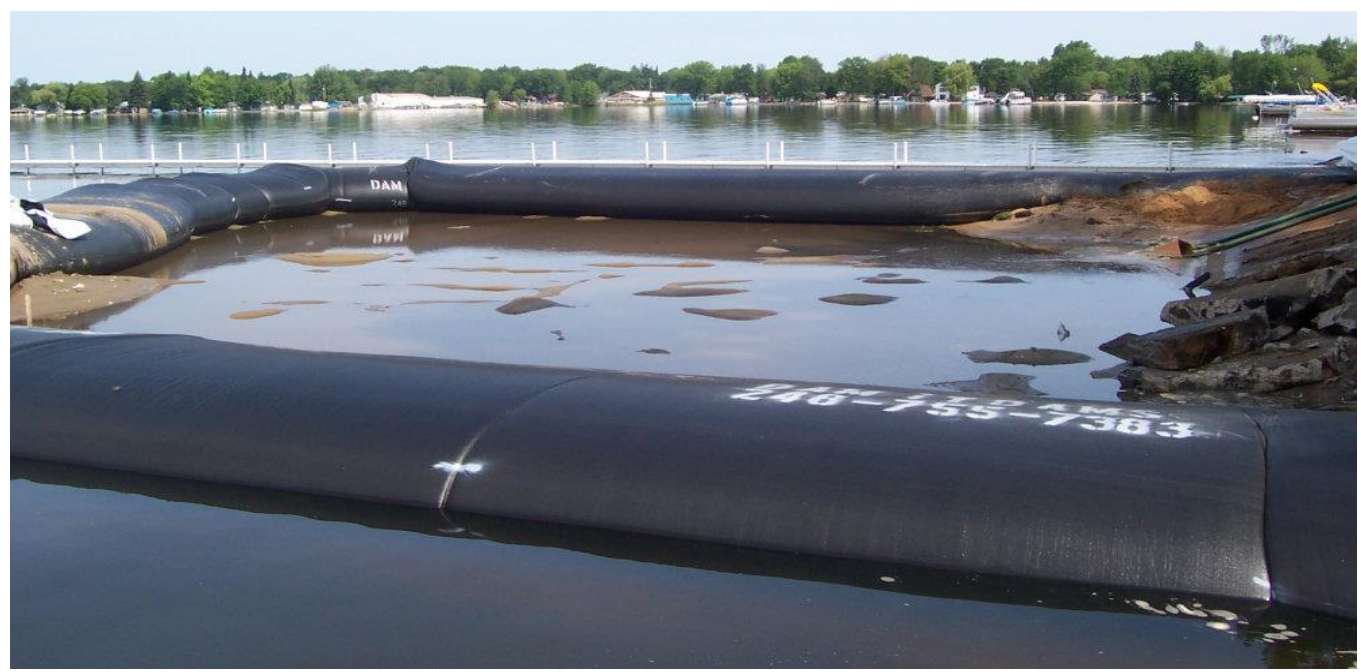

Figure 2-2: Water filled bladder (Dam-It-Dams, 2016). 
Repairs in-the-wet include the use of equipment like diving system which includes scuba diving and surface supplied diving, remotely operated vehicles and underwater tools (Michael J. Garlich, John E. O’Leary, Katherine C. Heringhaus, \& Thomas J. Collins, April 2010). In terms of the diving systems, a scuba diver has better mobility underwater due to light weight equipment and minimal surface support. Limited quantities of breathing gas and poor communication than surface supplied diving restricts usability of scuba diving to smaller underwater repair works. In comparison to scuba diving, surface supplied diving has a continuous supply of gas by means of supply line connected above water. There is also a continuous communication between the diver and his support crew along with the potential for video monitoring. This makes it suitable for repair works over longer durations. Water temperature, visibility and water currents are the major factors governing the efficiency of the diving systems. Extreme temperature reduces the workable time of diver and might also effect the properties of the repair materials. Visibility underwater is essential for proper and efficient implementation of repair work and can be limited due to heavy water currents or loose sediments. Currents exceeding 2.5 feet per second effects the stability of the diver and accuracy of placing the repair material (Michael J. Garlich, John E. O’Leary, Katherine C. Heringhaus, \& Thomas J. Collins, April 2010).

As an example, underwater repair of discharge ports at the Chickamauga Dam is completed with glass fiber reinforced polymer wraps using divers as the discharge ports were submerged and the lock couldn't be dewatered (Soti, 2014). The complete process from surface preparation to wrapping of FRP was done by divers (Figure 2-3) with additional help from audio and video monitoring teams (Figure 2-4). 


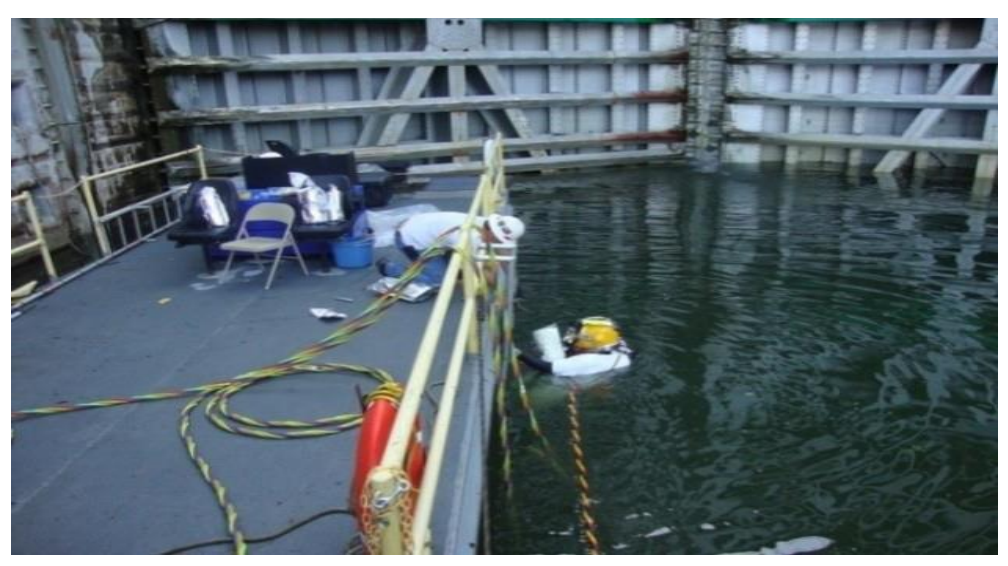

Figure 2-3: Diver, with support crew (Soti, 2014).

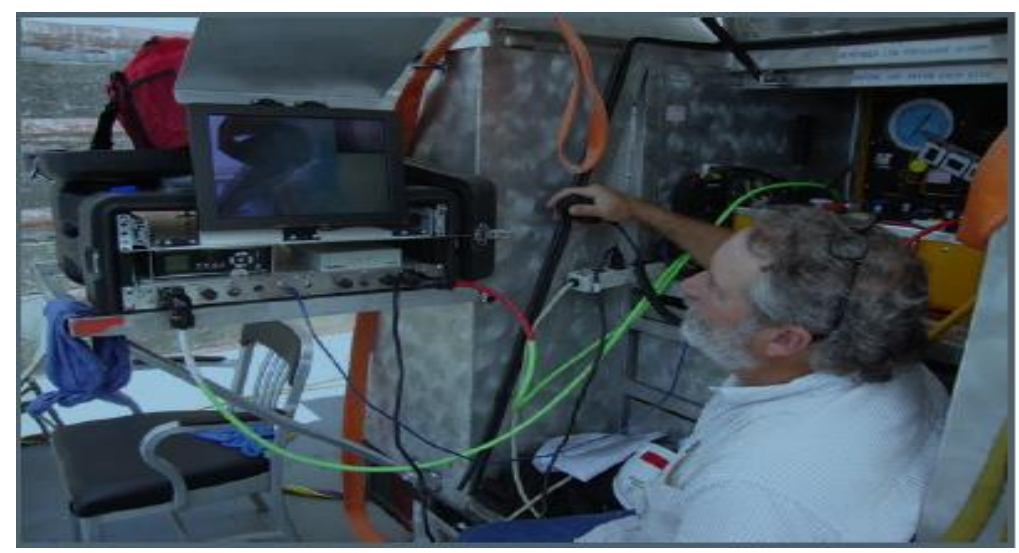

Figure 2-4: Audio and Video monitoring and communication setup (Soti, 2014).

Apart from the diving systems, remotely operated vehicles (ROV) are specially designed for underwater support. They are expensive and require specialized skill support from above water. These ROV's help in monitoring the video stream in real time during operations and also provide proper lighting to the repair site (Michael J. Garlich, John E. O’Leary, Katherine C. Heringhaus, \& Thomas J. Collins, April 2010). 


\subsection{Underwater excavation}

When the rehabilitation work is to be done below mudline, the soil is removed by the use various excavation equipment. The excavation equipment can be classified based on where it is used, either surface-based techniques (Figure 2-5) or underwater by diver (ABC Diving® , 2016) Figure 2-6 Diver Operated Equipment

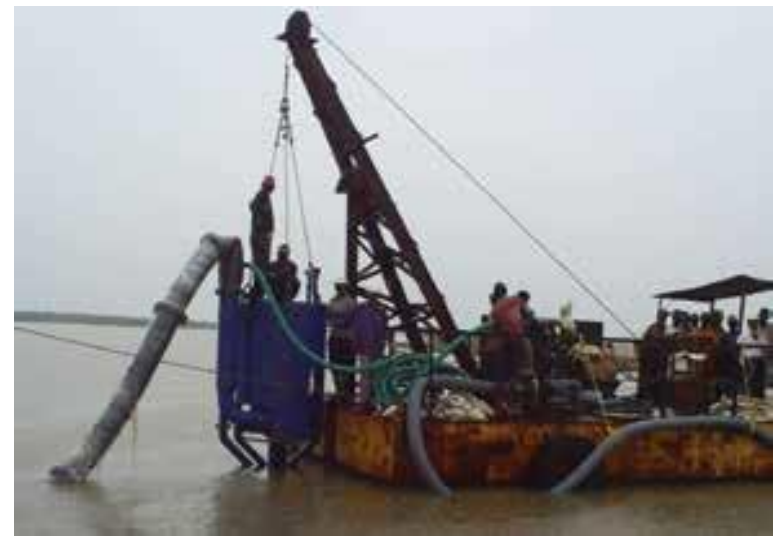

Figure 2-5: Equipment on Floating Platform (Michael J. Garlich, John E. O’Leary, Katherine C. Heringhaus, \& Thomas J. Collins, April 2010)

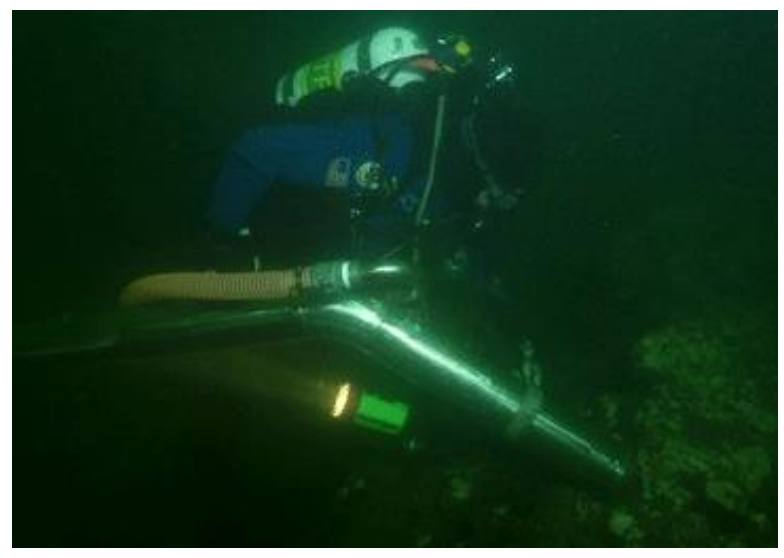

Figure 2-6 Diver Operated Equipment 
Surface based underwater excavation techniques include excavators, airlifts, jetting and dredging. (Michael J. Garlich, John E. O’Leary, Katherine C. Heringhaus, \& Thomas J. Collins, April 2010). The working depth of excavator is limited by the arm length of the excavator (Figure 2-7). These are mostly useful for the primary excavations where rough excavation work is good enough. Apart from excavators, hydraulic dredge pumps can be operated remotely and can be used for all water depths. It consists of a flexible hose with a suction pump attached to it. Sediments are stirred by a water jet (Figure 2-8) or agitator (Figure 2-9) fixed on a suction hose and are immediately drawn into pump (ABC Diving® , 2016).

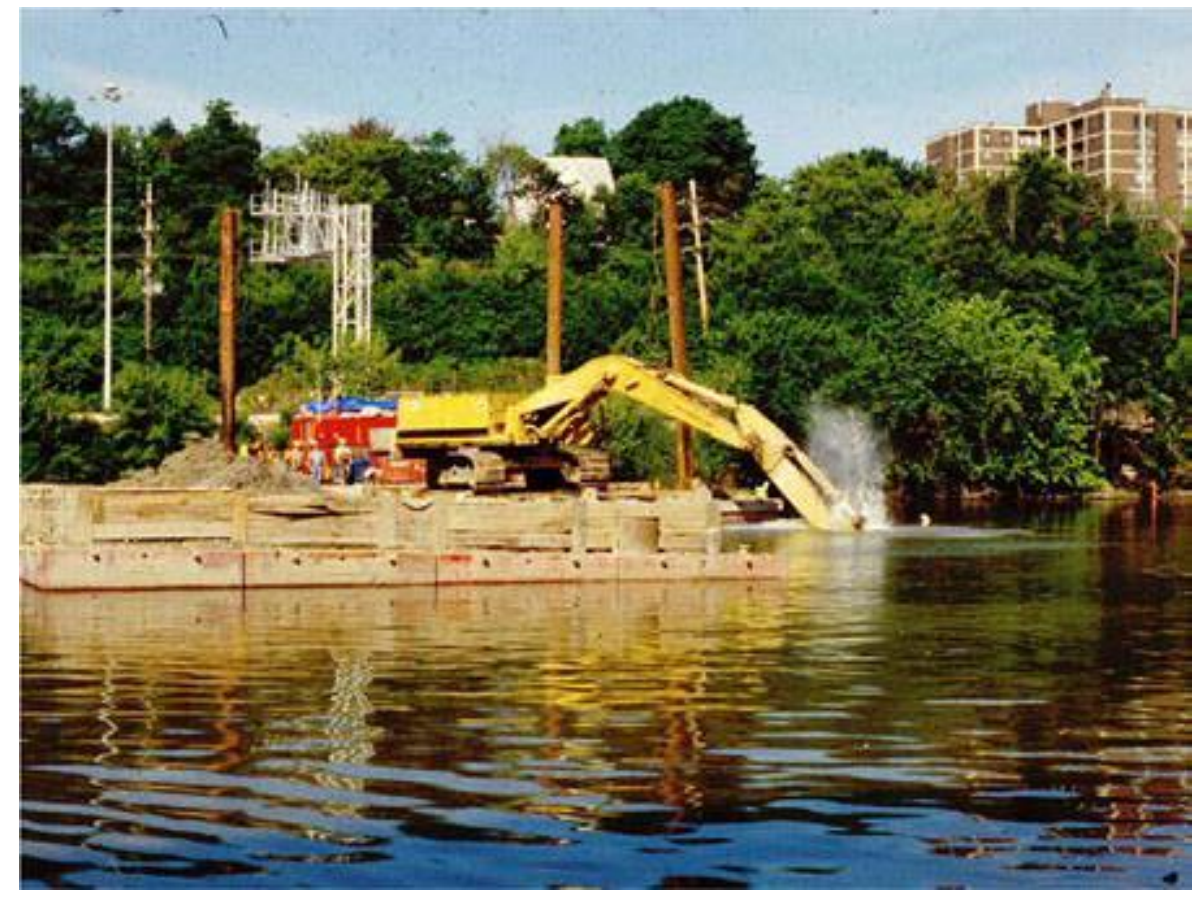

Figure 2-7: Excavator (ABC Diving® , 2016). 


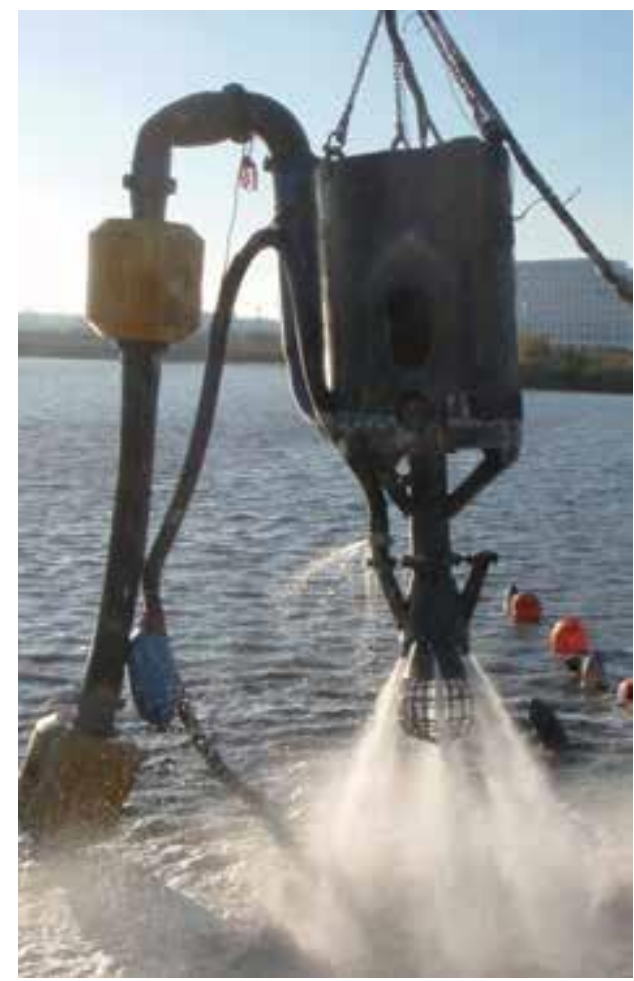

Figure 2-8: Hydraulic Dredger (ABC Diving® , 2016).

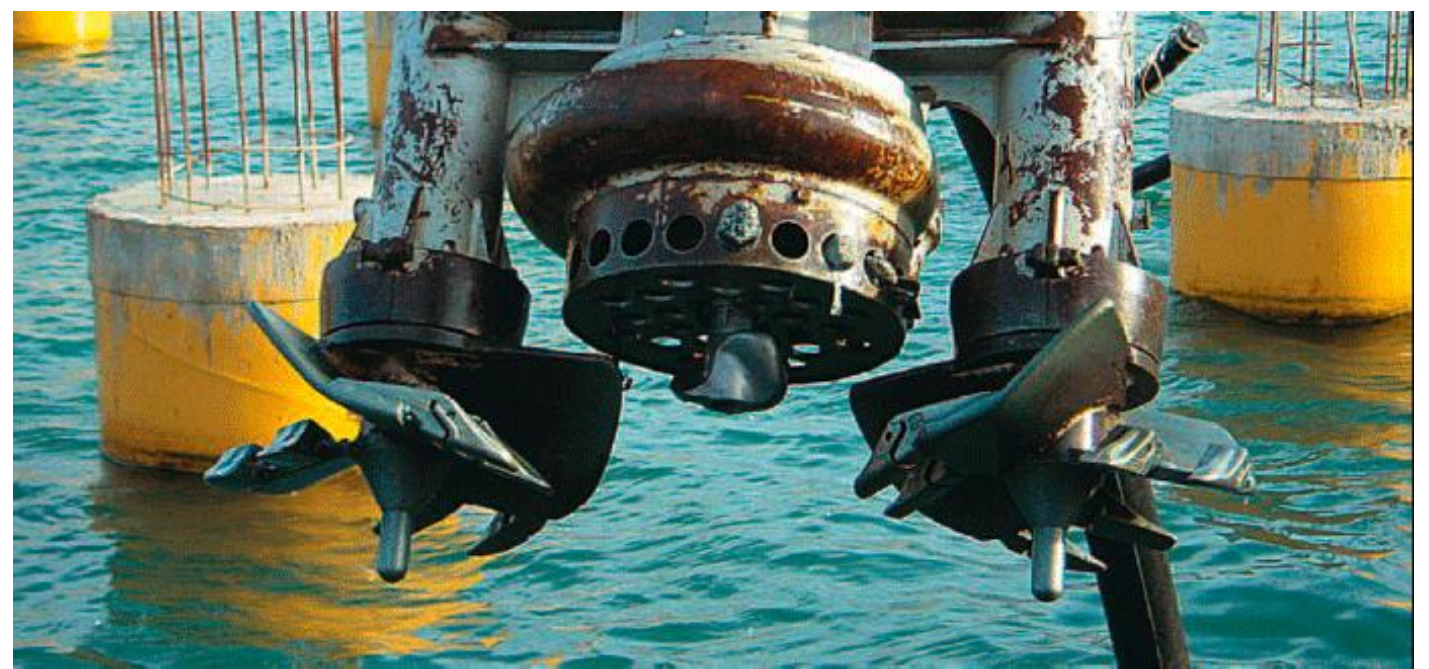

Figure 2-9: The suction pump with the agitators (ABC Diving® , 2016). 
The engineering principle of airlift operation is differential density between fluids. Induced air pressure causes bubbles to form in sediments which rise due to lower density. The air/water mix is lifted using suction. Airlifts are diver operated equipment mainly used for more accurate removal of material and are best suited for mud, sand, silt, clay and gravel.

In the case of jetting, water is pumped at very high pressure to the jetting tool from the pump located above water. The high pressure water breaks the sediments. It can be used at any water depths. This is mainly used for local displacement of sediments over short distances. This method decreases the visibility in water and increases turbidity. It is good for soft or loose mud, sand, silt or clay (Michael J. Garlich, John E. O’Leary, Katherine C. Heringhaus, \& Thomas J. Collins, April 2010).

\subsection{Underwater Repair Materials}

When it comes to underwater rehabilitation, piles are the most common substructures that need to be repaired (Michael J. Garlich, John E. O’Leary, Katherine C. Heringhaus, \& Thomas J. Collins, April 2010). They are subjected to physical damage and corrosion. The different kinds of piles in existing structures are steel H-piles, reinforced concrete pile, and timber piles. Appropriate repair schemes must be selected based on the extent of deterioration. Pile wraps and jackets are some of the available repair technologies

Fiber reinforced polymers are a good choice for structural repair systems because of their high strength to weight ratio (Gangarao \& P.V.Vijay, November 2010). They are available both in the form of wraps and jackets. Resins are available that can cure underwater and bond to wet concrete, which makes them a good option for underwater repairs. FRP wraps and jackets are discussed below: 


\subsubsection{Types of wraps and resins}

Fiber reinforced polymer composites are made of two constituent materials: fibers (organic/inorganic) and polymer matrices/resins. Of the two constituent materials, fibers are the primary load carrying elements. The matrix/resin transfers the load between the fibers and protects them against mechanical wear and tear and environmental conditions.

Fiber reinforced polymer products are available in the form of reinforcing bars, structural plates and shapes or wraps. Wraps have been widely used for rehabilitation of structural members as they enhance the strength, stiffness, ductility and durability when wrapped (Gangarao \& P.V.Vijay, November 2010). These composite systems consist of tightly woven fabrics and compatible resin systems that cure at ambient temperature to form composites. The fabrics are available with different kinds of fibers such as the glass, carbon and aramid fibers. The selection of the fabric and the arrangement of fibers are based on structural requirements, such as unidirectional when strength required in only one direction, multi-axial/ plain weave when strength required in both the axis. The resin system consists of two parts, resin and additive. Different kinds of additives can be added based on the property that needs to be achieved for that particular repair such as hardeners, plasticizers, fire retardants, viscosity control agents, antioxidants, UV protection, etc. (Gangarao \& P.V.Vijay, November 2010). The different types of FRP wrap systems are discussed in detail below. 


\section{FRP Wraps Systems can be separated into two types}

1. Dry fabric systems: For these wrap systems the fabric wrap is shipped dry to the site before being thoroughly saturated in the two-part resin system prior to installation. Such composite system can be cured at room temperature. The quality of the end product is mainly dependent on the skill of the person hand laying and squeezing of excess resin.

2. Prepreg composite system: A prepreg composite system consists of fabric reinforcement that is pre-impregnated with resin at factory and shipped to job site in sealed bags. (Figure 2-10).This system eliminates the need to wet-out the fabric at the job site, but they tend be more costly and have much more limited shelf life.

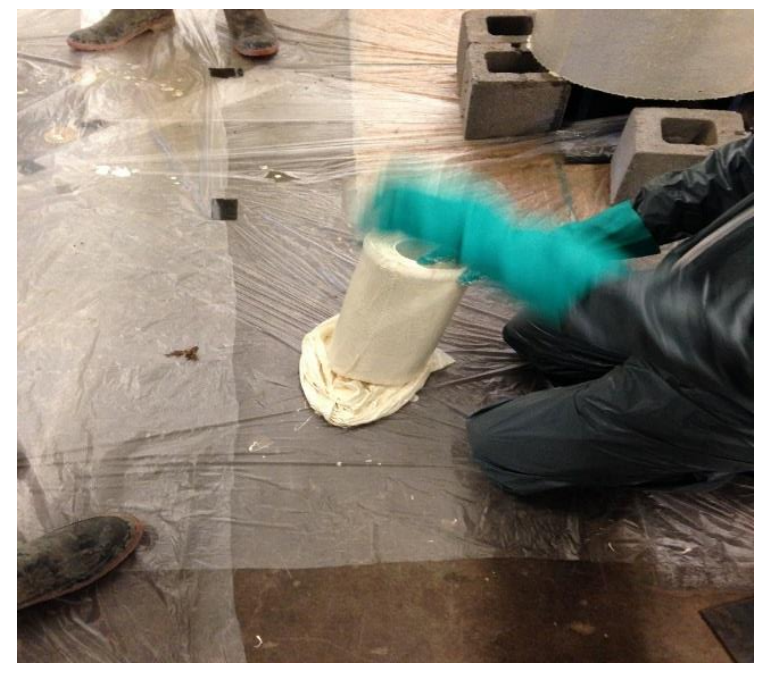

Figure 2-10: Pre-preg FRP system.

FRP wraps are available in glass, carbon, aramid fibers. Each variety of fiber has unique properties. This review only focuses on glass fiber as they are economical and corrosion resistant. 


\section{Glass Fibers:}

Glass fibers (Figure 2-11) are among the most versatile industrial materials in the reinforced polymer industry. These fibers exhibit high strength, flexibility, stiffness and other desirable properties such as corrosion resistance, chemical resistance or hardness can be obtained by changing the composition of raw materials when manufacturing. (Frederick T. Wallenberger, 2001).

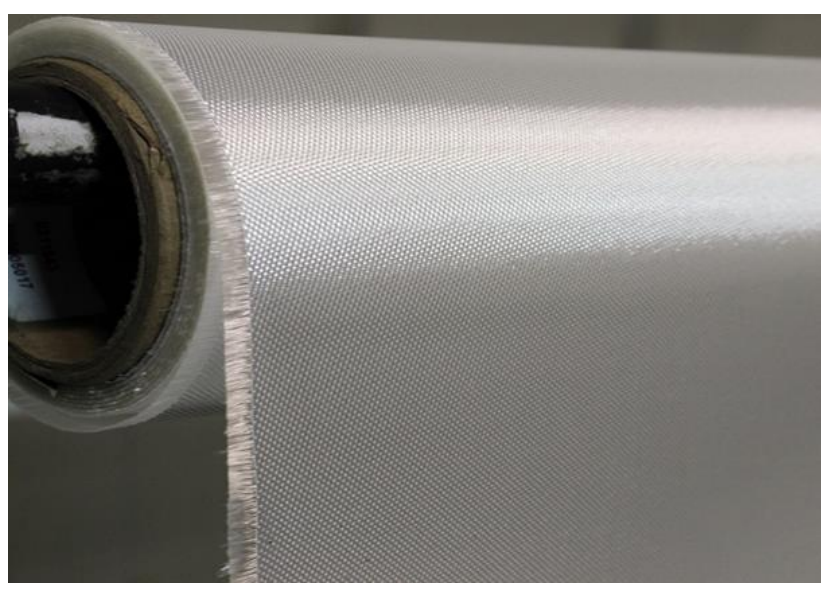

Figure 2-11 Glass Fiber fabric

\section{Resins:}

The resins fall into two categories thermosetting and thermoplastic. Most fiber reinforced polymer composites use thermosetting resin. Uncured thermosetting resins are in liquid state at room temperature (Gangarao \& P.V.Vijay, November 2010). During curing, the resin molecules form extremely strong bonds with one another and change state from liquid to solid. Thermoset means that once the resin is cured and forms into matrix it cannot be remolded or reshaped. At room temperature liquid resins are easy to work. They also exhibit excellent properties (Gangarao \& P.V.Vijay, November 2010). 
- $\quad$ Resistance to solvents and corrosives.

- $\quad$ Can withhold high temperatures and heat resistant.

- $\quad$ Can easily bond with fibers.

- Lower stress relaxation

But thermosetting resins have some limitations (Gangarao \& P.V.Vijay, November 2010)

- The strain-to-failure ratio is very low

- They have limited storage life.

Some of the common thermosetting resin include:

- $\quad$ Polyester Resin

- Vinyl Ester Resin

- $\quad$ Epoxy

- Urethane

Each resin has different properties. For example, urethane is good for underwater rehabs, while the epoxy is good for corrosion resistance.

Thermoplastic resin becomes soft from solid state upon heating. Once the process of making a composite is done, they return to solid state (Gangarao \& P.V.Vijay, November 2010).

The advantages of thermoplastic resins are (Gangarao \& P.V.Vijay, November 2010)

- $\quad$ They have high impact strength.

- $\quad$ Higher strain-to-failure.

- Shorter fabrication time. 
- $\quad$ Storage life is much higher at room temperature compared to thermosetting resins.

\subsubsection{Fiber Reinforced Polymer Shell/Jacket}

FRP composites are installed as wraps via hand lay-up in the field or manufactured into shells in the factory. The shells have an axial/vertical slit so that they can be opened and placed around the columns with ease during installations. One such product is the Simpson Strong-Tie Fx-70 jacket (Figure 2-12 ). They have a tongue and groove joint as shown in Figure 2-13. Once FRP jacket is placed around the column they can be fastened using bolts and adhesive along the slit. They not only add strength but also act as in-situ form work for grouting purposes. Based on the strength required we can add required number of layers of FRP wrap around the shell.

The advantages of FRP shell/ jacket are:

- They act as in-situ form work, very useful for underwater repairs.

- The FRP jacket is a corrosion free system which prevents deterioration.

- It is UV-resistant. 


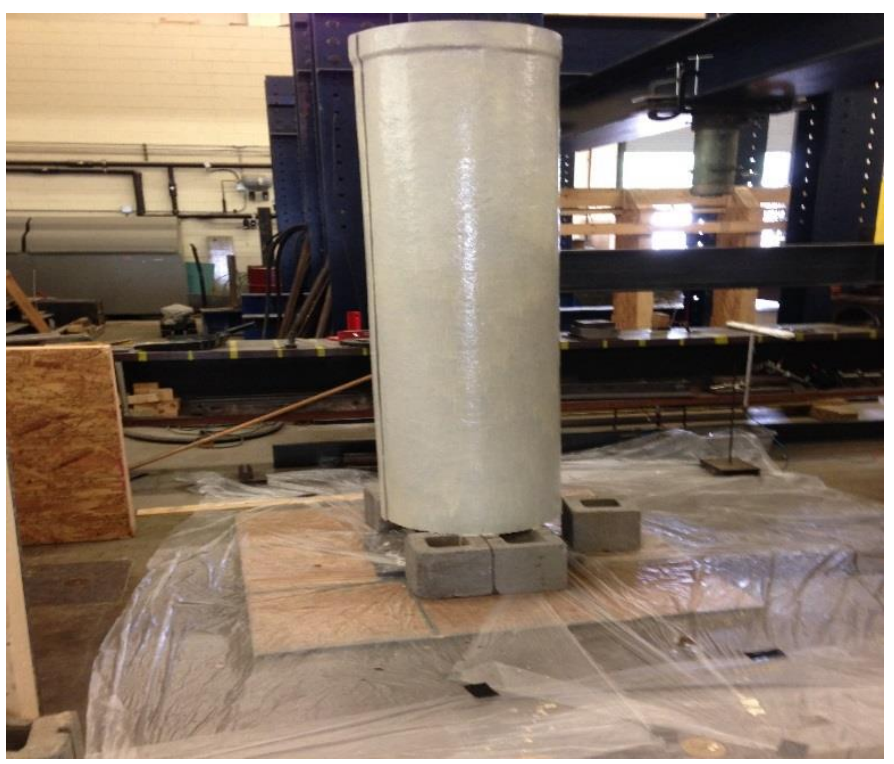

Figure 2-12: FRP shell/jacket.

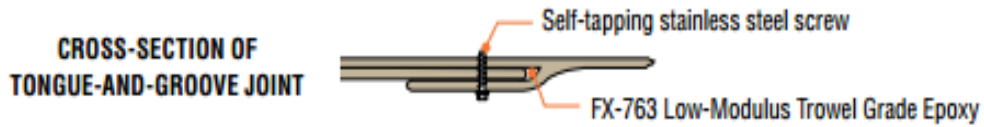

Figure 2-13 : Tongue and Groove Joint (Simpsons Strong-Ti, 2016) 


\section{Chapter 3. Fiber Reinforced Polymer Shell-Auger System}

\subsection{Introduction}

The proposed new technology regards a repair system for encapsulating corroded or deteriorated steel or concrete piles of bridges and other structures, including the ones below the mudline, using a novel fiber reinforced polymer shell with an auger attachment. This repair system is easy to adopt around the existing deteriorated pile without disturbing the structure.

The fiber reinforced polymer shell is a circular-cylindrical shell with a longitudinal slit from top to the base of the jacket. The auger attachment is made from two steel semi-circular rings that join to form a circular ring. The FRP jacket is placed around the pile and the longitudinal slit is sealed to form a shell column. The two semicircular auger rings are placed around the pile and adjoined to the FRP shell column at the bottom (Figure 3-9). Then fiber reinforced polymer wrap is wound around the column to provide additional strength. Force is applied on the shell causing the column to bore into the earth's surface, i.e. below mudline

The novelty of this repair system is boring of shell around an existing pile without separate excavation work. This repair system is aimed at not only reducing the cost and repair time but also the environmental and safety issues of the repair methods. The key components of this repair system are FRP shell, auger attachment and the augering mechanism which are discussed in detail below. 


\subsection{Fiber Reinforced Polymer shell}

Fiber reinforced polymer shells/ jackets are one of the repair systems that have been widely in use for underwater repair and rehabilitation. The FRP shells not only provide protection but also reinforce the structure.

In this novel rehabilitation technique of structures underwater and below the mudline, the fiber reinforced polymer shell plays the major role. The FRP shell is the main structural member, but still light and easy to install in the field. The longitudinal slit makes it easy to open and place it around the existing piles. Fiber reinforced polymer shells (or jackets) are pre-cured shells made typically using multi-axial, stitched, nonwoven fiber fabrics. These shells come in a variety of shapes, lengths and wall thicknesses, though this work focuses around circular shells to facilitate the augering.

The circular shape of the FRP shell allows for an auger to be attached and the entire shell to be twisted into the ground without effecting the existing pile. It is also crucial for transferring the applied torque/ vertical force to the auger attachment and serves as an in-situ form work for the further rehabilitation process. This eliminated the need to use barrier systems or divers. Based on the depth of boring the length of the shells can be selected. The FRP shell need not be a single member for the whole length; they can be multiple cylindrical shells circumferentially attached to one another. 


\section{Wrapping around the shell}

The strength of the FRP shell alone is not sufficient to resist the installation stresses and provide confinement strength for the concrete repair. In order to have sufficient strength, additional layers of fiber reinforced polymer wraps are applied around the FRP shell. Number of layers required depends on the strength required.

The FRP wrap should be applied around the FRP shell after the shell is placed around the pile and sealed along the longitudinal slit. The wrap can be applied to strengthen any circumferential joints in the shell. The use of prepregs and underwater-compatible wraps would speed the installation time of the wraps. These additional wraps not only provide additional strength and protection they increase the stiffness to withstand applied torque and downward thrust. 


\subsection{Design of Auger Attachment}

Existing excavation techniques are not only costly, inefficient and unsafe when working around against existing piles but also have environmental issues if done in-the-wet due to the displaced soil. The displacement or deposition of theses excavated sediments should be dealt very carefully. The design of the auger attachment would overcome the existing challenges as the system would cut a circular trench around the pile with minimal disruption to the surrounding soil. The remaining soil inside the shell can be removed with hydraulic augering in the contained shell environment.

The auger attachment is the driving component of the new repair system to enable the shell to penetrate below the mudline. The challenge is to auger it around an existing pile or substructure. The design of auger attachment should be such that it can be assembled around the existing pile i.e. in the form of split rings. It should also have enough clearance from the existing structure for a smooth boring into the soil. As it cannot be retrieved, it should be economical.

The auger should be strong enough to with stand different soil conditions ranging from soft soils to small rip-rap, and different types of augers can be made for different types of soils. The bonding of FRP shell and the auger attachment is crucial as the torque is transferred through the bond. Three different types of auger attachments were developed herein for different soil conditions: 1) cutting edge auger, 2) excavating teeth and 3) bullet type teeth.

Each type of auger is attached to the FRP shell via an auger ring. The auger ring consists of two semi-circular members as show in Figure 3-1. These semicircular members, when joined, form a circular ring. These members are manufactured from steel with a 3/4 inch thickness and 4 inches in height (Figure 3-1). The auger ring has a circular recess of about 1" high on the outer 
side surface to receive the base of the FRP jacket. This ensures that the outer surface of the ring and of the FRP shell are on the same circular plane, thus the auger ring does not disturb the soil outside of the FRP shell. The recess also has provisions for bolting/riveting at the overlapping portion of FRP jacket and ring attachment.
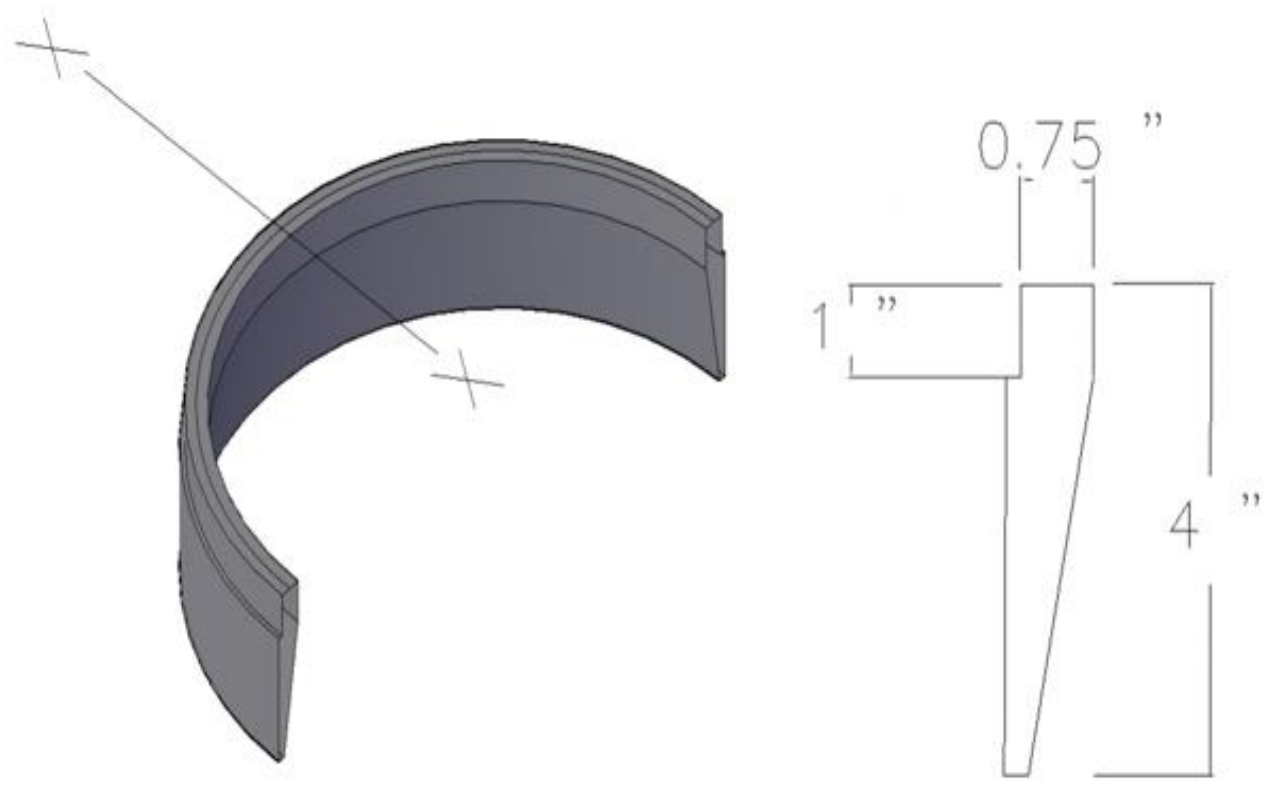

Figure 3-1: Arched member with the cross-section. 


\subsubsection{Cutting Edge Auger}

The first design of auger is simply using the auger ring as a tapered cutting edge. The interior surface of the auger attachment is angled starting from an inch below the top surface extending till to bottom surface, thus forming a cutting edge as shown in Figure 3-2. This is a very simple design obtained from the principles of pile driving. The reduced cross-section at the bottom causes the bottom edge to cut into the soil as it is pushed down. This particular design pushes the soil inward as it bores down. Inward cutting edge is advantageous over an outward cutting edge, because the pushing the soil inward allows it to be easily captured inside the confined shell using hydraulic augering. This limits the environmental disturbances of contaminated soils and pushes soil into the empty cross-sectional volume of the shell.

As we apply torque and vertical force on to the shell, the cutting edge auger attachment drives the shell into soil. For this kind of auger attachment greater vertical downward force should be applied compared to torque. This attachment is mostly useful if the soils profile at the work site is loose/ soft clay without much of boulders. It is estimated that this particular attachment design costs about $\$ 300-400$ for the rolled steel auger attachment of $3 / 4$ inch thick and 4 inches high.

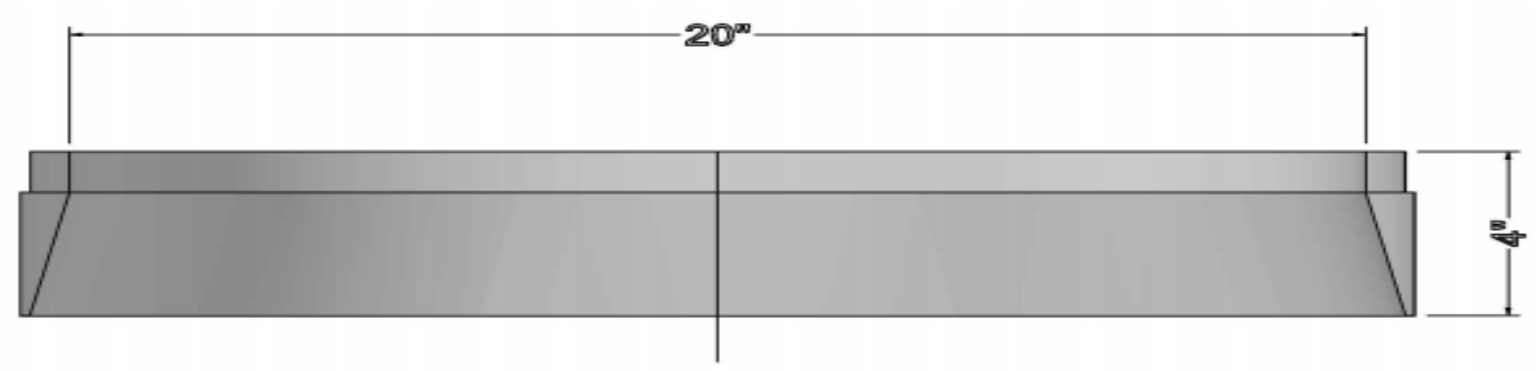

Figure 3-2: Arched member front view. 


\subsubsection{Excavating teeth}

As it will be shown in Chapter 4, the cutting edge design was not very successful in penetrating during laboratory tests. As it was mostly driven by vertical force, and the applied torque has hardly any effect. In order to make use of the torque, screw threads were attached to the auger ring, along with the addition of excavating teeth to help clear away the soil.

The screw threads consist of metal strips of 1" wide cut from a thin metal sheet in the form of an arc as shown in Figure 3-3. Six of such arcs were cut and placed at equal distances on the outer surface for the metal ring. They are placed in such a way that one end of the arc is at the bottom of the 3" metal ring and the other end is at the top of metal ring thus making it an inclined screw on the outer portion of the shell.

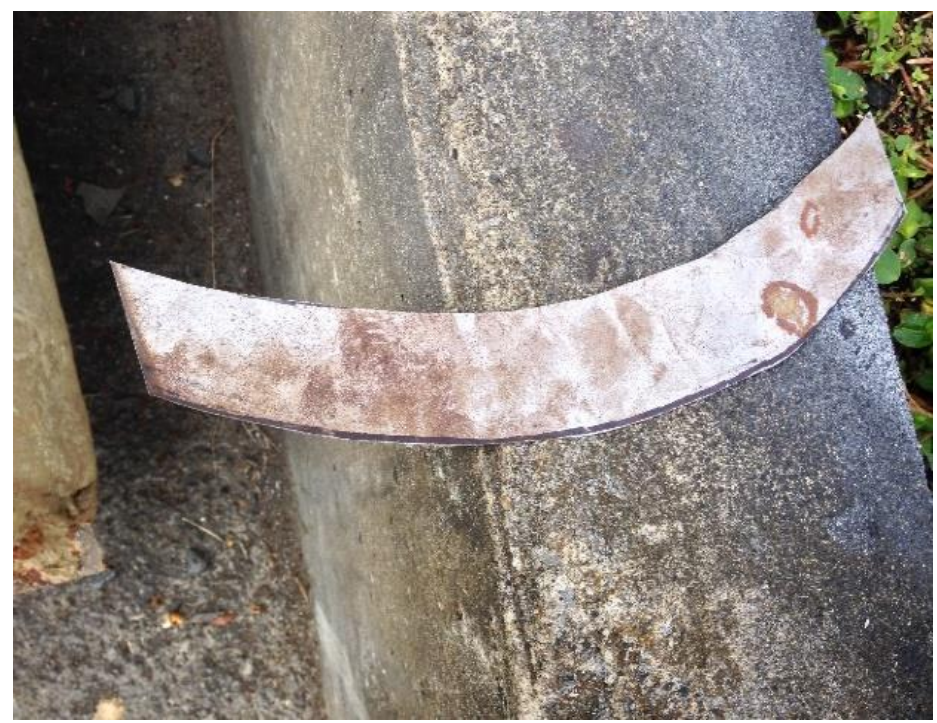

Figure 3-3 Arc metal strips 


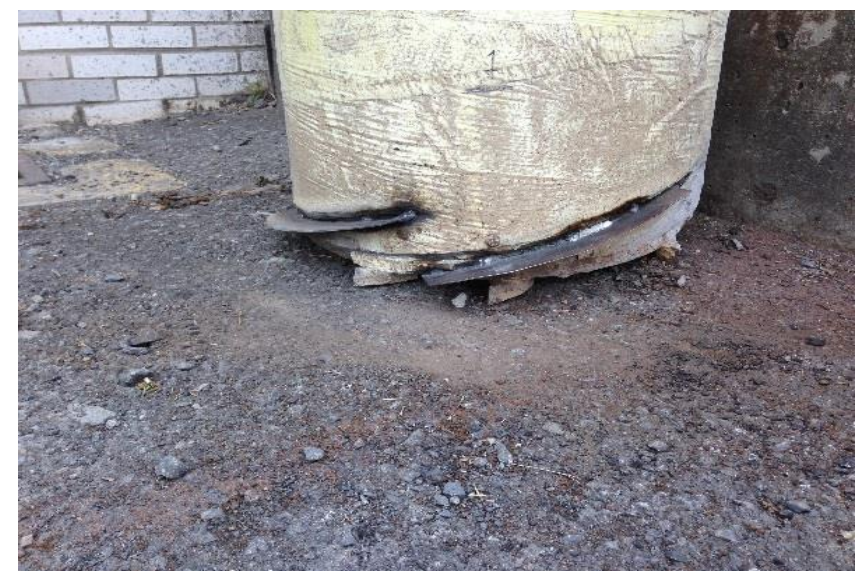

Figure 3-4: Additional Helical attachments to Auger.

The excavating teeth consist of specially designed teeth can be attached at the bottom surface of the auger ring. Each tooth is made from a 1"x1"x3" block of rectangular hardened steel as shown in Figure 3-6. The teeth are be designed in such way that applied torque helps to clear the soil away from the bottom of the auger ring.

Two surfaces that will come in direct contact with soil are made inclined in this rectangular block. The bottom surface (1"x3") is made at an inclination of $15^{0}$ as shown in Figure 3-7. Having a pointed edge on the bottom, the teeth always stay penetrated in the soil. One of the 1"x1" sides is made inclined at $45^{\circ}$ as shown in Figure 3-8. This helps in cutting through the soil with the sharp edge facing towards the direction of augering. The teeth are welded in such a way that all the sharp edges (1"x1") are pointing to the direction of application of torque and extend outside the ring as shown in Figure 3-9. This design is good for rotational augering as the tooth shovels the soil giving good clearance to the FRP shell. This cutting edge can push aside the medium pebbles but cannot with stand larger rocks. The teeth extend slightly outside the auger ring to make more clearance 
around the ring as it bores down reducing the friction between the soil and the shell, but not so far as to inhibit the screw threads. The total cost of this design is estimated to be around $\$ 350-450$ for 12 teeth attachments and 6 screw attachments.
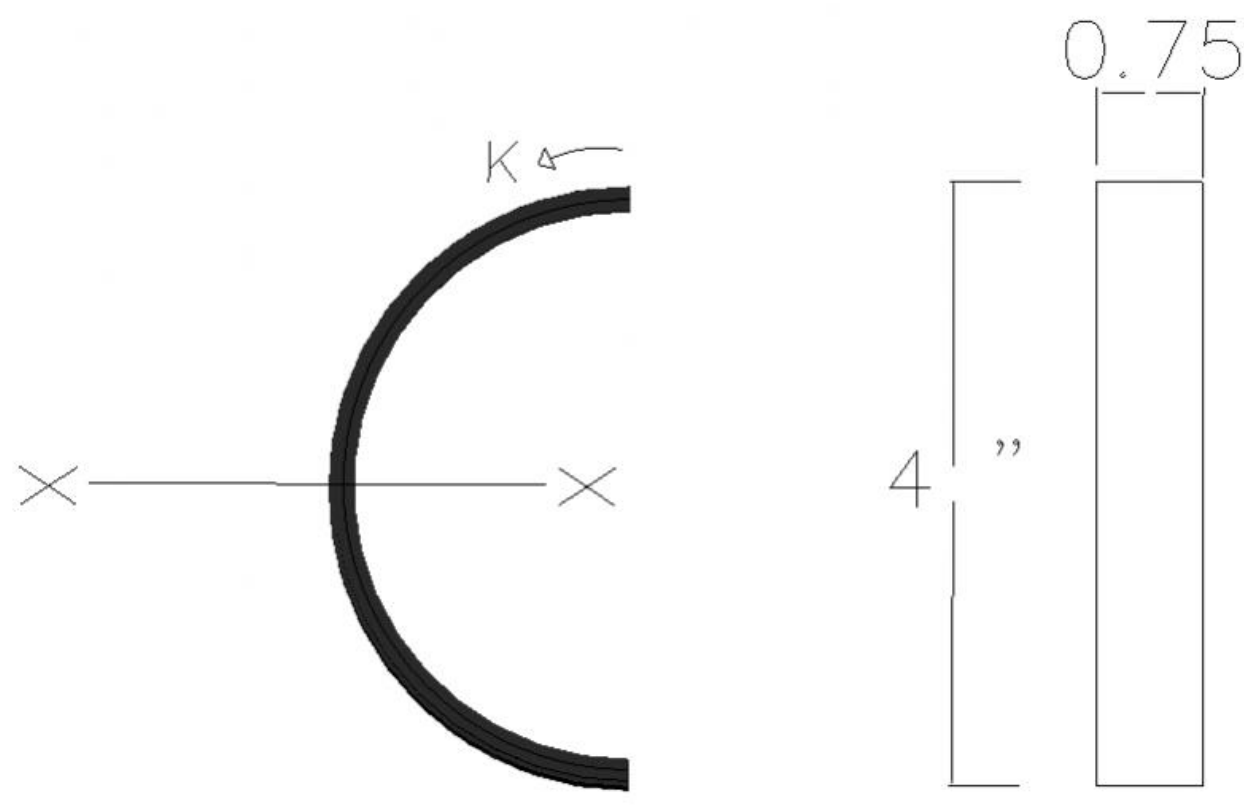

Figure 3-5: Cross section of the semicircular ring.
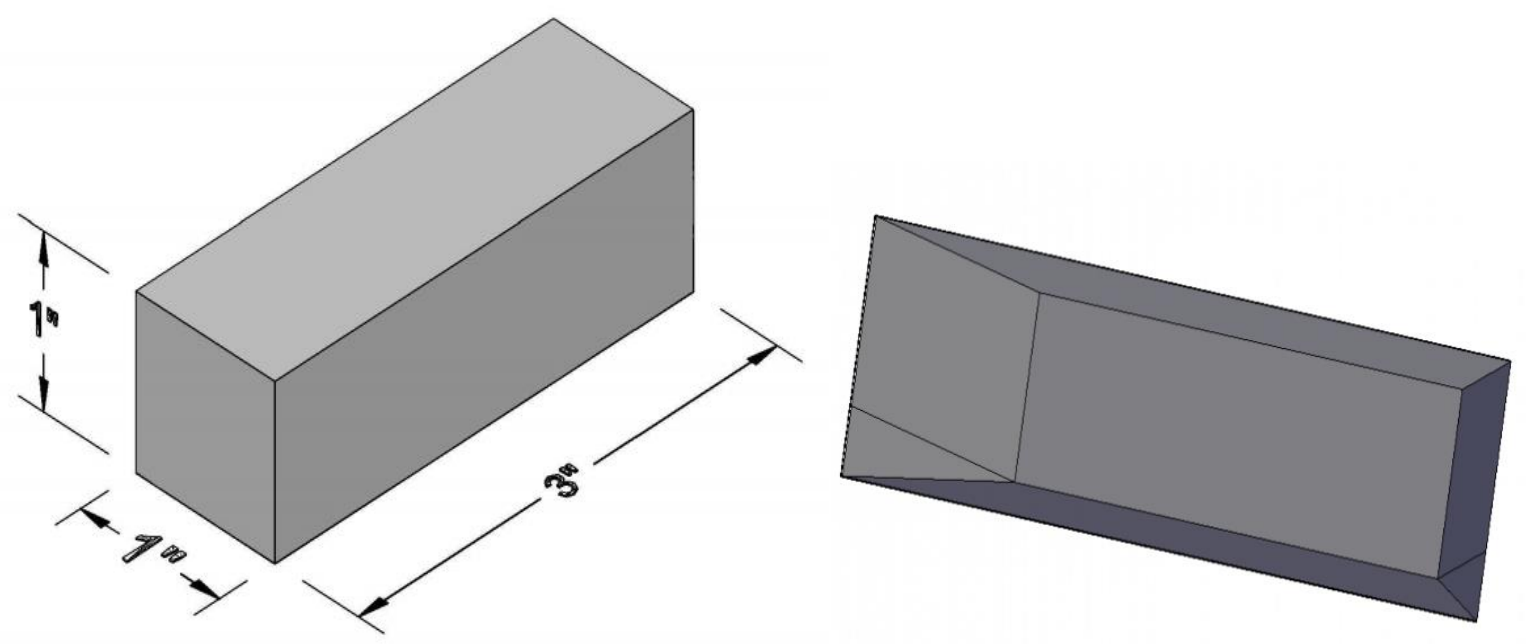

Figure 3-6: Rectangular block from which the cutting teeth is made. 


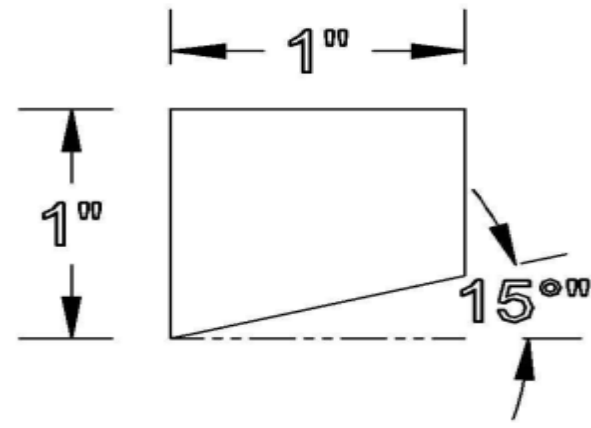

Figure 3-7: Cutting teeth (bottom side).

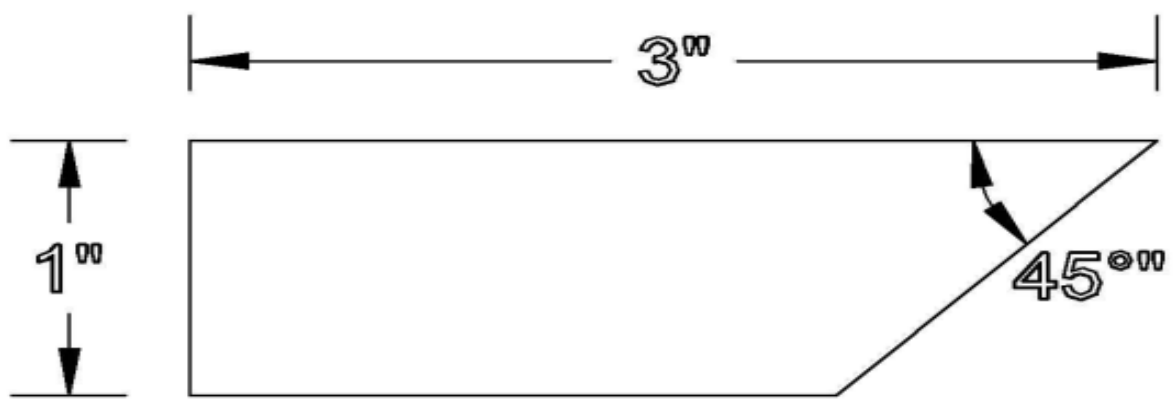

Figure 3-8: Cutting teeth with the cutting edge.

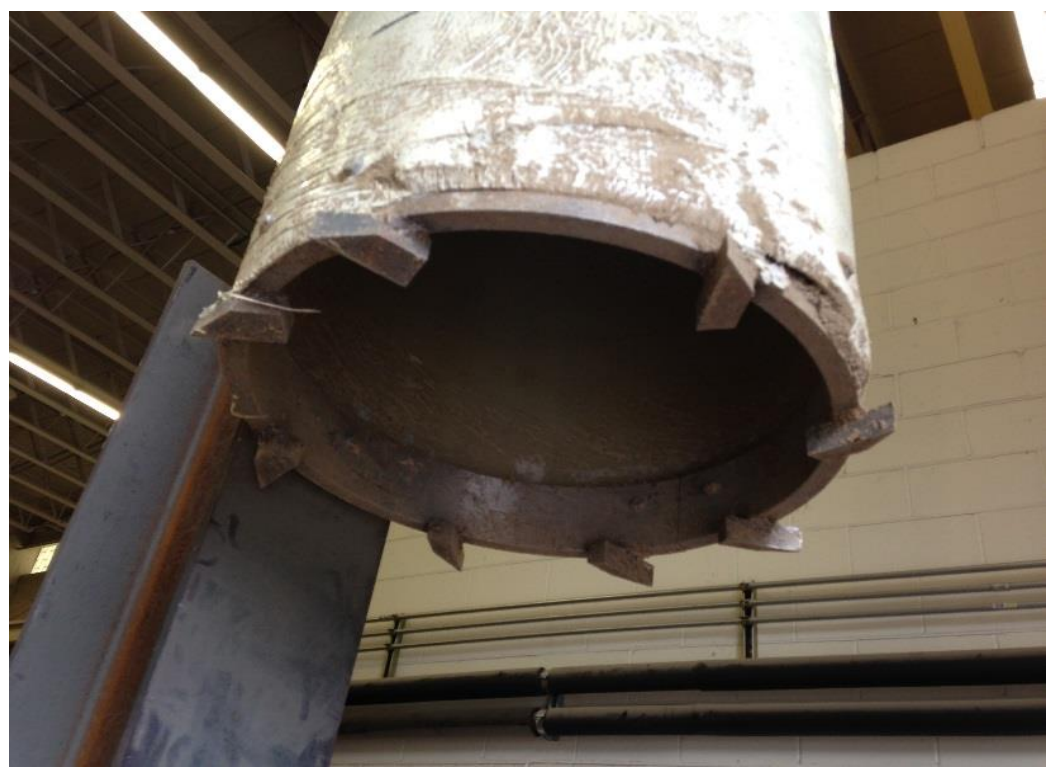

Figure 3-9: Auger attachment with cutting teeth. 


\subsubsection{Bullet type teeth}

The final design took advantage of bullet teeth that are already in wide use for cutting into soil and rock. In place of the excavating teeth, conical bullet teeth with block attachments were used, as shown in Figure 3-10. The bullet teeth and the block attachment have an interlock mechanism, and the block attachment can be placed at various angles as per requirement of the excavation. The blocks are welded along the bottom circumference of the ring in groups of three. One facing away from the ring (i.e. to the outer surface), one facing along the ring, and one facing towards the inner surface of the ring. A total of 12 teeth were welded (Figure 3-11) on the bottom as shown in Figure 3-12. This kind of arrangement of teeth ensures better mobility of the auger even in pebbles or rip rap as they push the rip rap aside.

The bullet teeth with block attachment stand about 3 inches in height when attached at the bottom. The force from the downward thrust is transferred by the FRP shell on to these 12 bullet teeth attachments. The force is transferred as 12 point loads on to the soil and application of downward thrust pushes the teeth into soil. This concentrates the vertical load which amplifies the effect of it and the applied torque. The bullet teeth and block attachment costs bring the total cost of the auger to $\$ 450-500$.

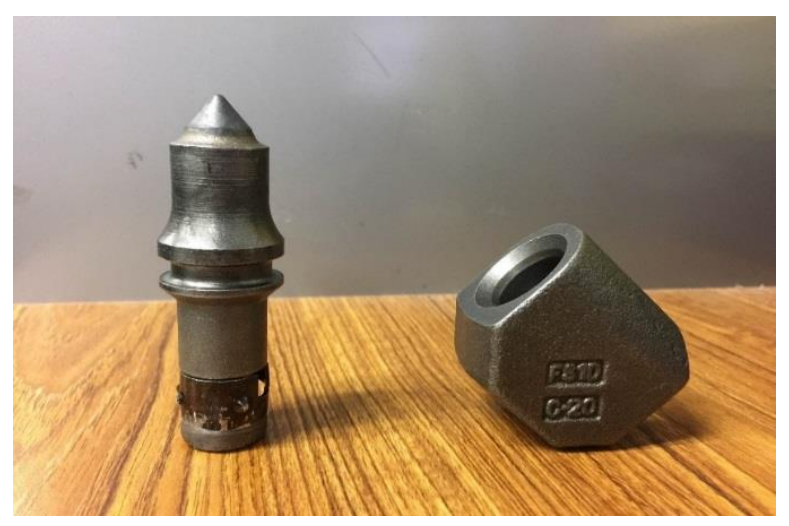

Figure 3-10: Conical bullet teeth and block holder. 


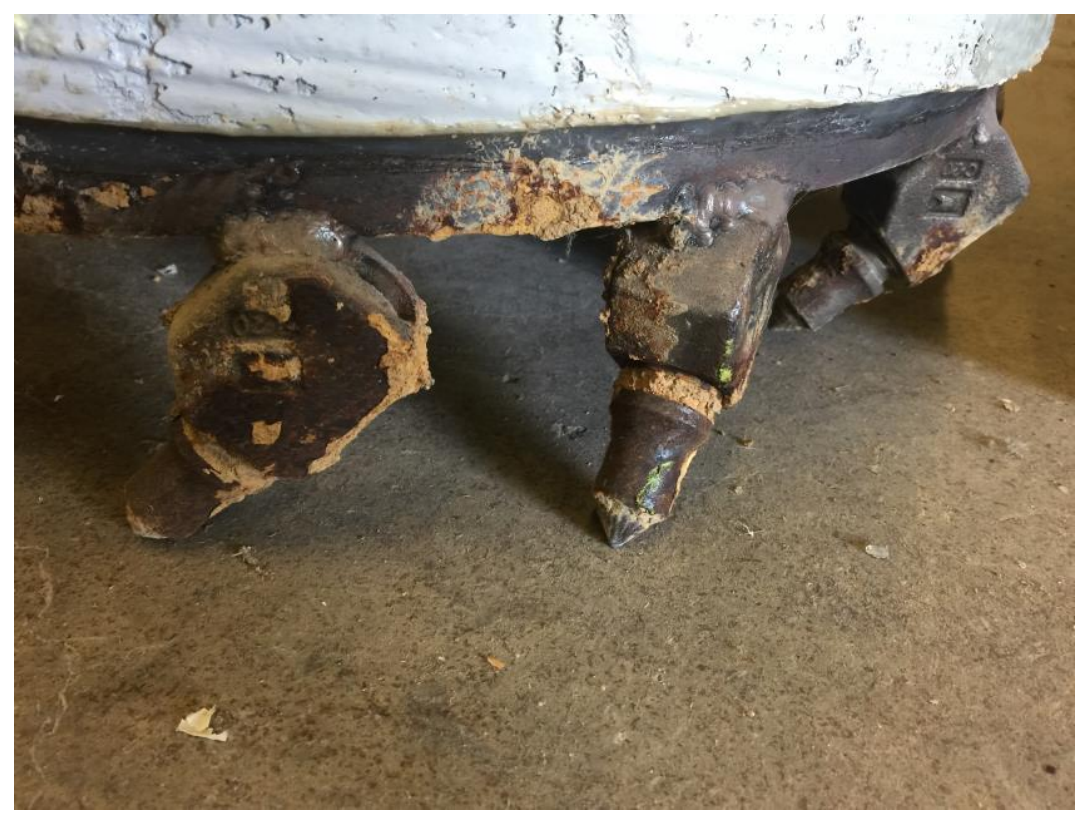

Figure 3-11: Bullet teeth with the blocks attached to the circular ring.

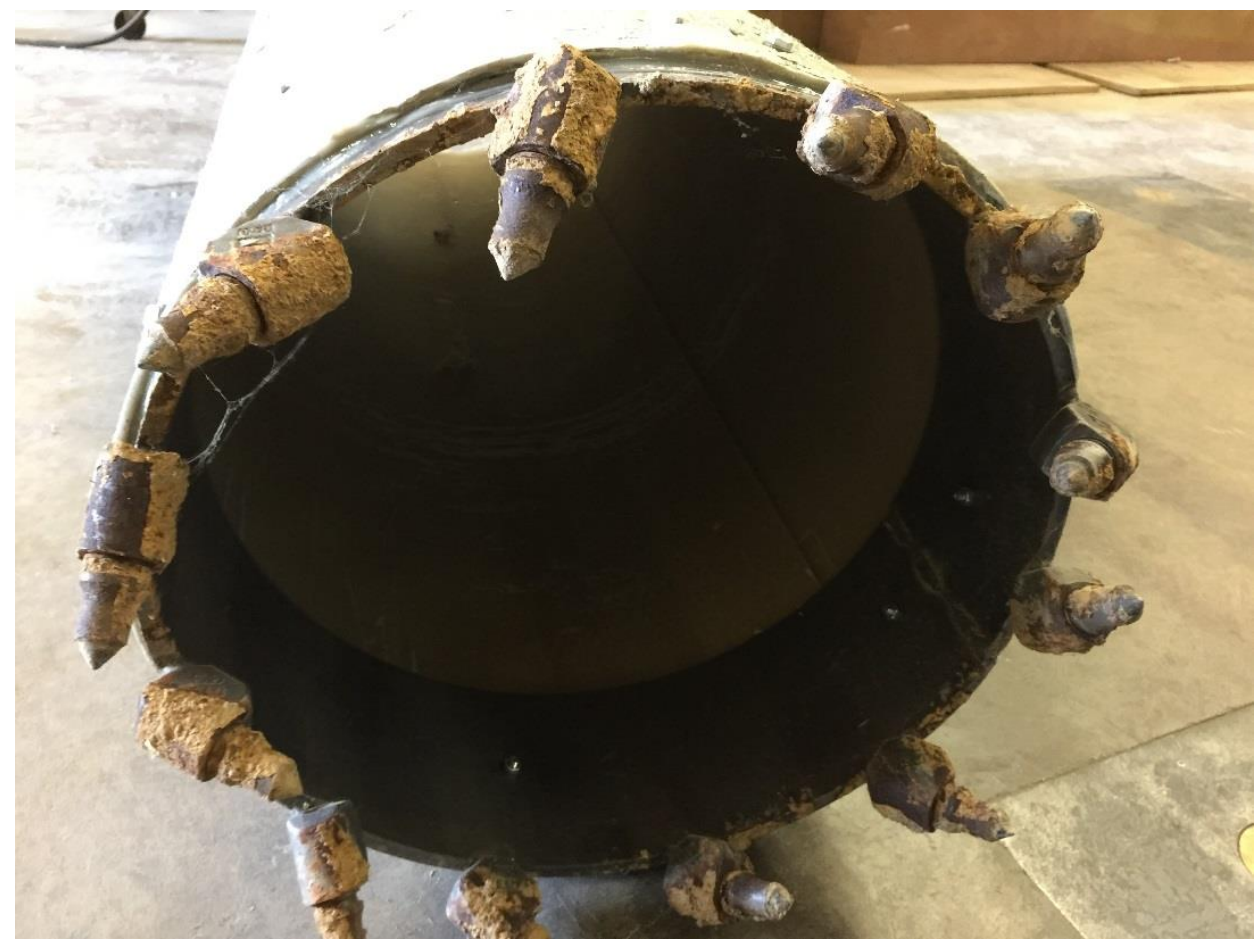

Figure 3-12: Auger with bullet teeth. 


\subsection{Augering Mechanism}

The auger mechanism is the mechanical method to apply vertical or rotational force on the column shell causing it to bore into the ground to the designed depth. The augering mechanism can be broadly classified into either manual driving or mechanical driving. Based on the site conditions, the appropriate augering mechanism can be chosen. Both types require the application of torque and either vertical loads or vibration to drive the auger into the soil.

\subsubsection{Manual Torque Application}

Manual augering mechanisms involve two people manually applying the force to generate the torque and require good reachability and accessibility around the repair site to maneuver. Manual methods are suitable for shallow depths and soft soils, where there will not be much of resistance to bore the shell-auger system into the soil. For the laboratory investigation as disclosed in Chapter 4, split metal rings adjustably secured around the shell were used to apply the torque. At least two cylindrical steel tubes (say 6 feet in length) are welded on each ring, adequately spaced apart to allow one or more persons to grasp the tubes and generate torque on the shell (Figure 3-13). This process is limited as the length of the tubes requires ample space around the pile, space that may not be available with adjacent piles. Alternately, a chain wrench (Figure 3-14) can be 
looped around the column shell to generate torque. The chain wrench can be disconnected and reattached multiple times, thus minimizing the space requirements.

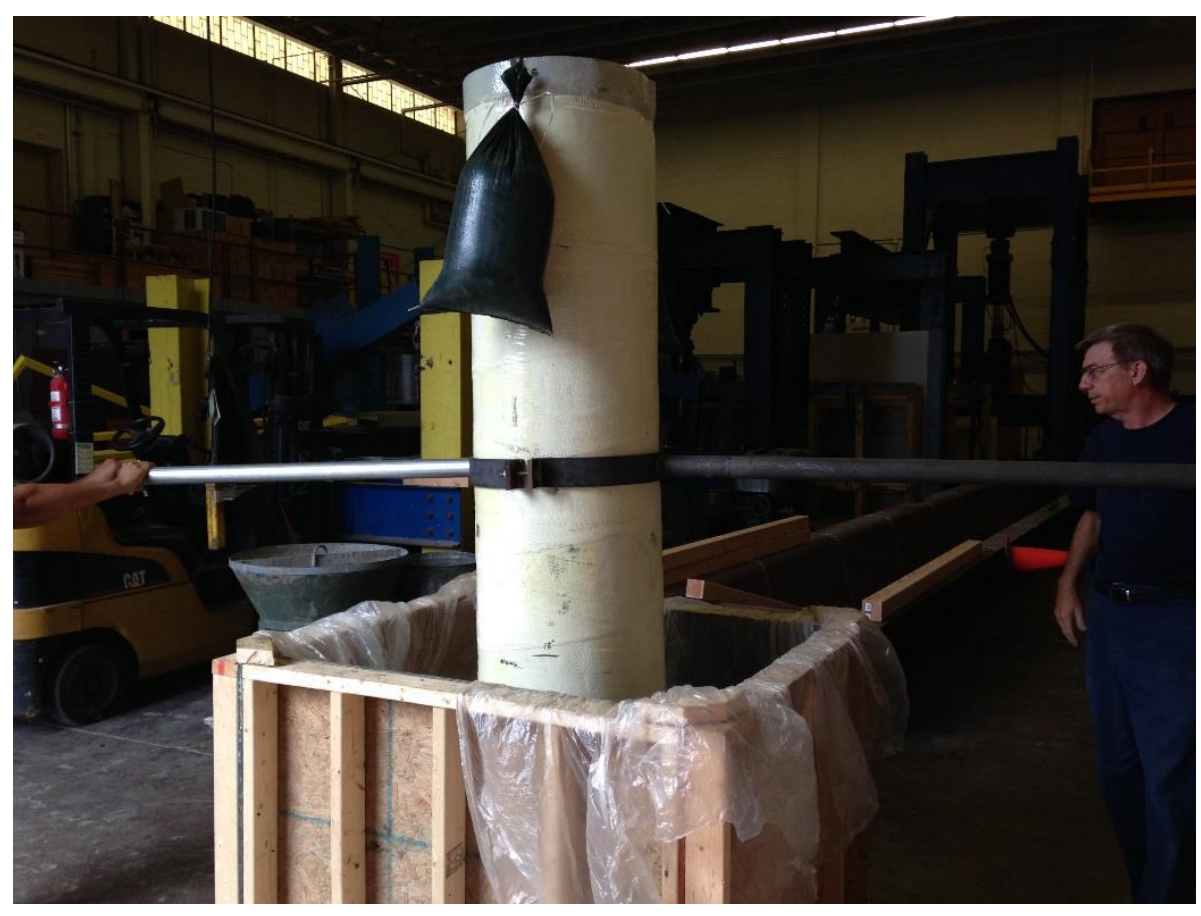

Figure 3-13: Manual application of torque.

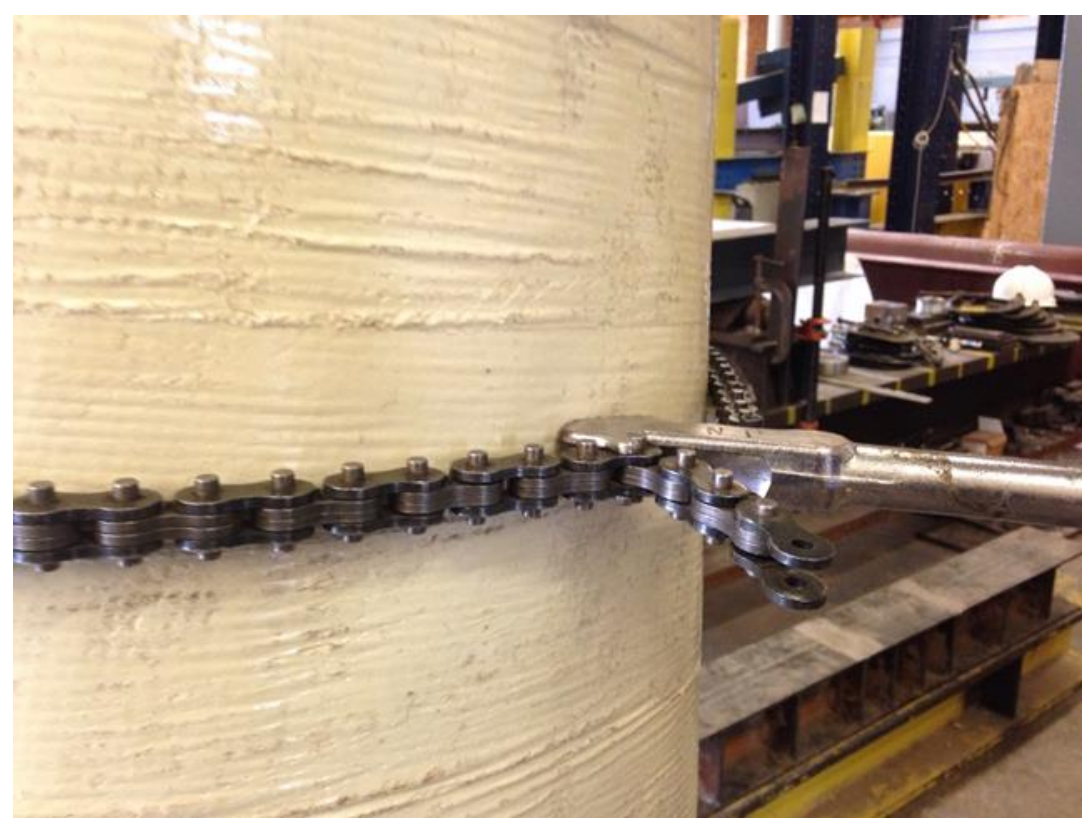

Figure 3-14: Chain Wrench. 


\subsubsection{Mechanical Torque Application}

It is anticipated that in typical field work the manual application of torque would not be sufficient to drive the shell into the soil. There are a number of ways in which the torque can be generated with the use of mechanical equipment. The equipment not only makes the job easier but can also work under varied soil conditions, compared to the manual methods best suited for soft soil conditions.

The scope of use of mechanical equipment at the repair site is governed by the effect of mechanical equipment on the existing structures, as it is expected that the torque would be resisted by anchoring to the existing structure. Mechanical torque could be applied using a gear reduction motor in place of normal motor to have controlled boring of the shell. The force from the motor could be transferred to the shell via a chain attached to an auger ring with teeth mounted to the top of the shell or via a looped cable running around the shell. The application of torque on the shell would require a counter acting force in the opposite direction of application of torque to maintain the shell centered around the pile, which could easily be accomplished with 2 motors. If a cable is used, care should be taken while applying torque mechanically by using protective padding at the point of application to distribute the force into the shell and prevent local failure. The maximum applicable torque for the system tested in Chapter 4 is discussed in Appendix B. Based on the material strength the maximum applicable value of torque is $143922 \mathrm{lb}-\mathrm{ft}$, which is high. However

others factor have to be considered while calculating potential failure modes and arrives at FRP thickness. Some of the potential failure modes are: local crushing, local buckling, joint seam strength, bond between FRP and augering system etc. Other factors such as local failure and more specific to the method of torque application can also be considered in future studies. 


\subsubsection{Vertical Load/Vibration}

Apart from the application of torque, the addition of vertical loads or vibrations would help the augering process to go smoothly. A uniformly applied vertical load not only helps in augering but also provide stability to the column shell. A simple passive method to apply vertical load is to directly hang sand bags of required weight symmetrically on the shell (Figure 3-15). The load application through sand bags is cost effective but limited by the total amount of load that can be applied. A uniform distribution of load should be maintained to prevent eccentric augering. Active methods to apply vertical force can be obtained by using a hydraulic jack or a vibratory driver. The maximum applicable vertical load is discussed in Appendix B. The maximum vertical load capacity of the shell-auger system used in this study is 143 kips. But if we are using a hydraulic jack other factors such as the capacity of the existing structure should be considered in deciding the maximum value.

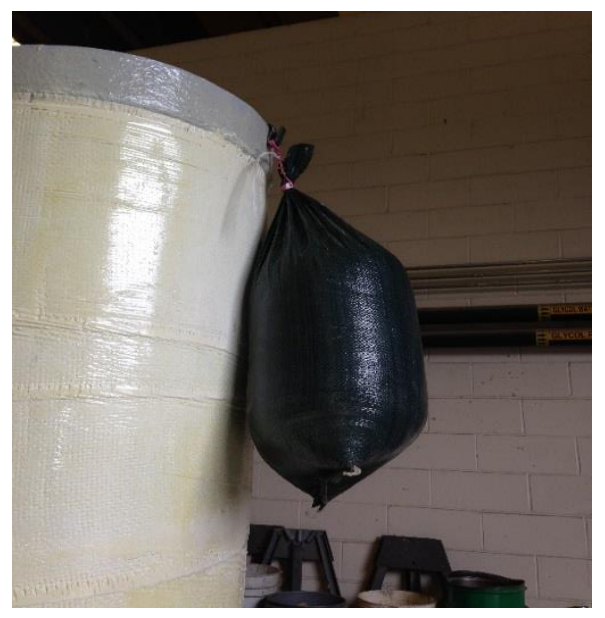

Figure 3-15: Sand bags Used to apply Vertical loads. 


\subsubsection{Hydraulic Jack Mechanism}

A hydraulic jack mechanism is one of the active methods of applying force onto the shell. A pair of semi-circular plates which together form a circular plate is placed on top of shell, so that the force coming from the jack on to it can be applied uniformly on to the shell. On top of this plate would be a series of ball bearings, with hydraulic jacks on top. The ball bearings would allow the shell to rotate while the vertical force is being applied. The hydraulic jack presses against the underside of the existing structure.

The load application should take into consideration the strength and existing stresses in the existing structure, including global and local stresses due to the concentrated jacking forces. The major constraint with the hydraulic jack mechanism is proper anchorage for the hydraulic jack/driver around an existing column or pile. There should be enough space for the FRP shell and the hydraulic jack, but additional shells can be added after augering if enough space exists over the waterline. The applicable loads and capacity of the structure should be calculated with a factor of safety and the clearance should be checked before adopting a hydraulic jack. A sample calculation of capacity of using an existing structure is shown in Appendix B. The dead weight of the section of East Fork Bridge was calculated to compare with the maximum applicable vertical load derived based on the material properties. The dead weight of the bridge span came out to be 109 kips which is less than the maximum applicable vertical load calculated 143 kips. For this particular case even though the material can take the load, the application of that load against the existing structure can cause uprooting of the structure. 


\subsubsection{Vibratory driving}

Upon vibrating the shell, the soil particles around the shell get excited. This reduces the friction between the soil and shell temporarily thus the shell can be driven into the soil. (Figure 3-16).

The commercial vibratory-drivers are mainly classified as

- Hydraulic vibratory-drivers

- Electrical vibratory-drivers

The hydraulic vibratory-drivers are more feasible, as they are lighter in weight, and allow driving-frequency adjustment.

Hydraulic vibrators consist of four main parts (Kenneth Viking, May 2002) (Figure 3-17):

- A suppressor housing,

- Elastomer pads,

- An exciter block,

- A clamping device. 


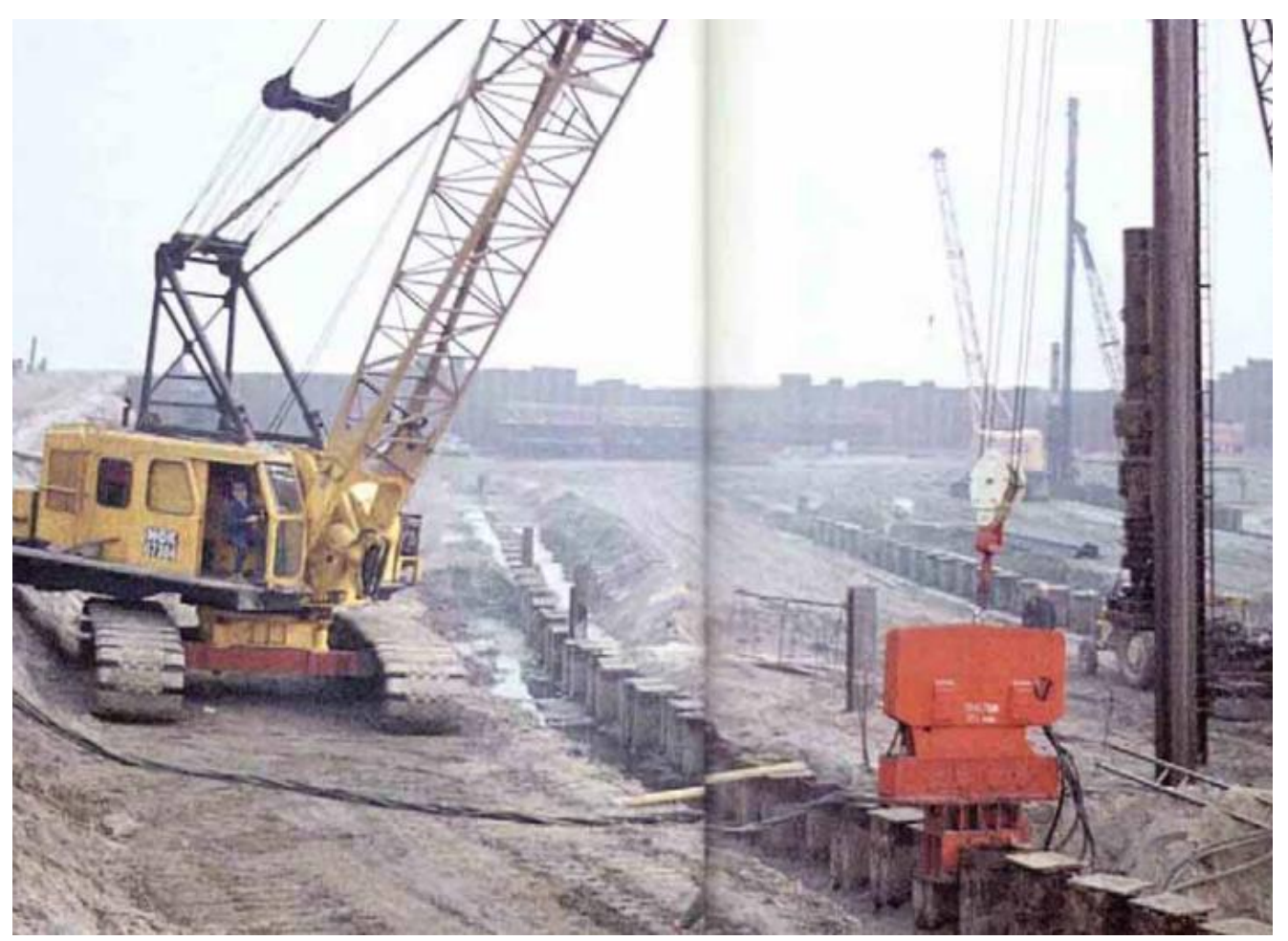

Figure 3-16: Hydraulic vibratory (ArcelorMittal, 2004)

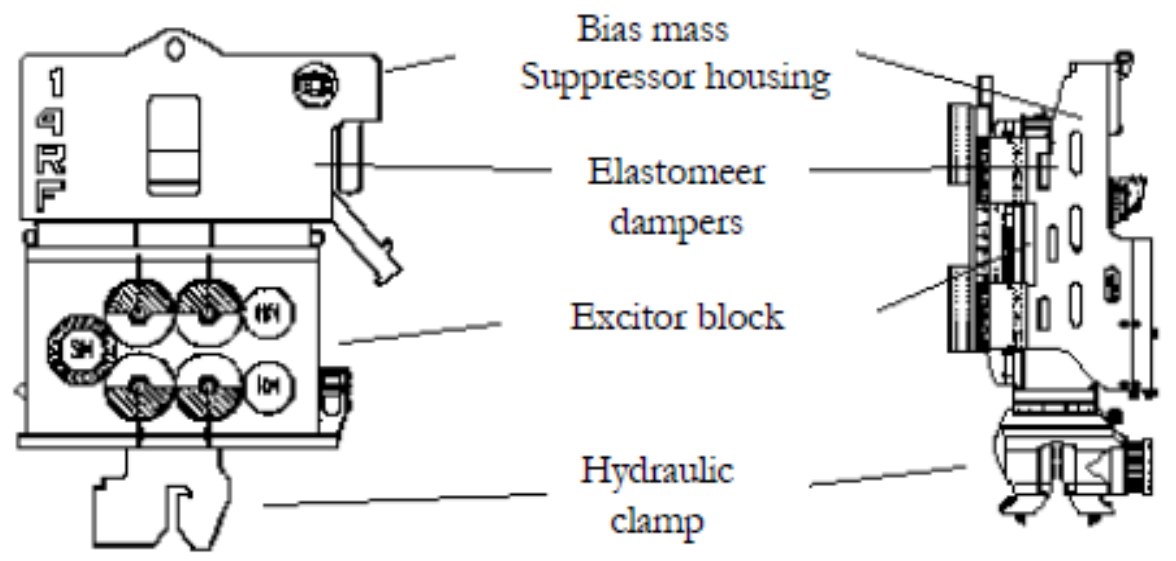

Figure 3-17: A free hanging vibrator (left) and leader-mounted vibrator (right) (ArcelorMittal, 2004) 


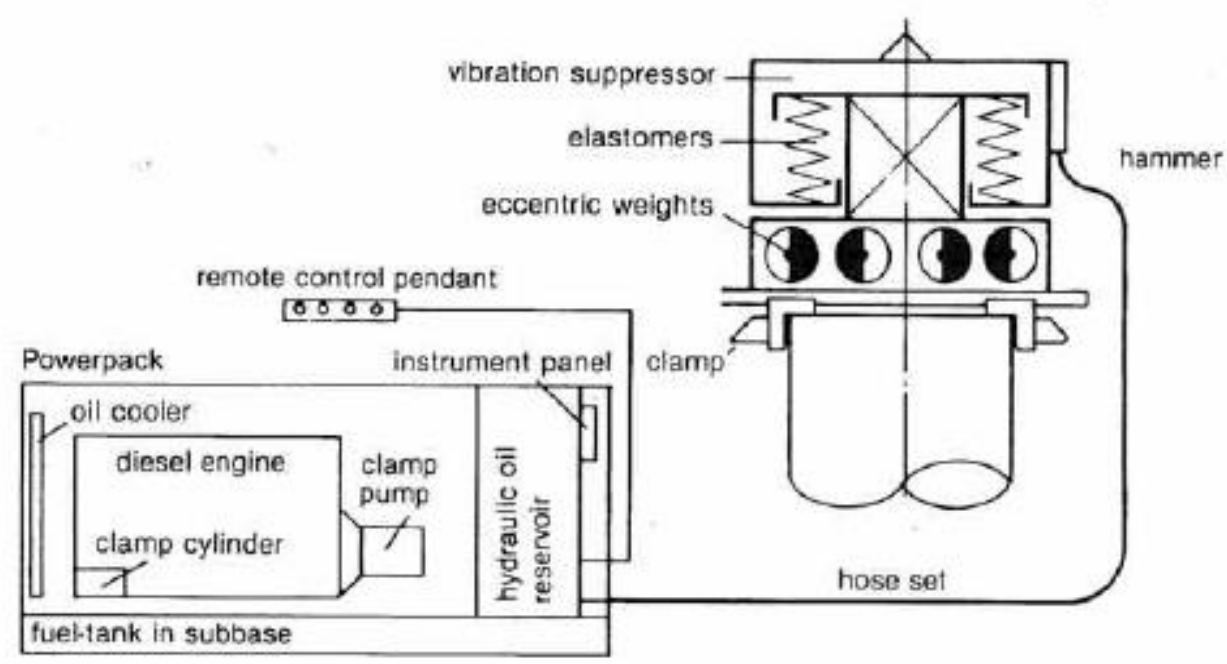

Figure 3-18: Principle of operation (Kenneth Viking, May 2002)

As summarized in (ArcelorMittal, 2004) hydraulically operated clamps attached to the shell transfer oscillating movements (Figure 3-18). Vibration driving works best in non-cohesive soils or gravel, especially when they are water-saturated, whereas artificially dewatered sands are very resistant to vibratory driving

\subsection{Flushing of Soil}

Once the FRP shell with the auger attachment is driven into the soil, the next major task is the removal of soil inside the shell at the bottom as the shell bores down. Hydraulic augering is the simplest way to remove these soils as it uses pressurized water to turn the soil into a slurry that can be pumped out of the shell. Removal of the soil during augering would aid in the augering process, so long as access into the shell is possible and the hydraulic pressure can be controlled to keep it contained in the shell. 


\section{Chapter 4. Laboratory Testing}

\subsection{Introduction}

This chapter discusses in detail the materials used; the test set up and how the test is carried out in the Major Units Laboratory at West Virginia University. The main objective of this testing is to know the effectiveness of the augering attachments used in this novel rehab technique with the fiber reinforced polymer jacket-auger system, to study the difficulties faced during the test run and to overcome those difficulties in a laboratory setting.

\subsection{Materials}

\subsubsection{Fiber Reinforced Polymer Shell/Jacket}

Fiber reinforced polymer shell jacket (Figure 4-1) is the major structural component of this rehabilitation process. For this testing, the Simpson Strong-Tie Fx-70 jacket was chosen for evaluation. These jackets are made out of high strength fiberglass in a polymer matrix and are inert, corrosion free. They have a tongue and groove seam (Figure 4-2) along their length, allowing the jacket to be opened for installation about the piles and sealed when in place about the pile.

Table 4. 1: Technical properties of FX-70 (Simpsons Strong-Ti, 2016)

\begin{tabular}{|l|l|}
\hline Water Absorption & $1 \%$ max \\
\hline Ultimate Tensile Strength & 15,000 psi-minimum \\
\hline Flexural Strength & 25,000 psi-minimum \\
\hline Flexural modulus of Elasticity & 700,00 psi-minimum \\
\hline Barcol hardness & $45+/-7$ \\
\hline
\end{tabular}




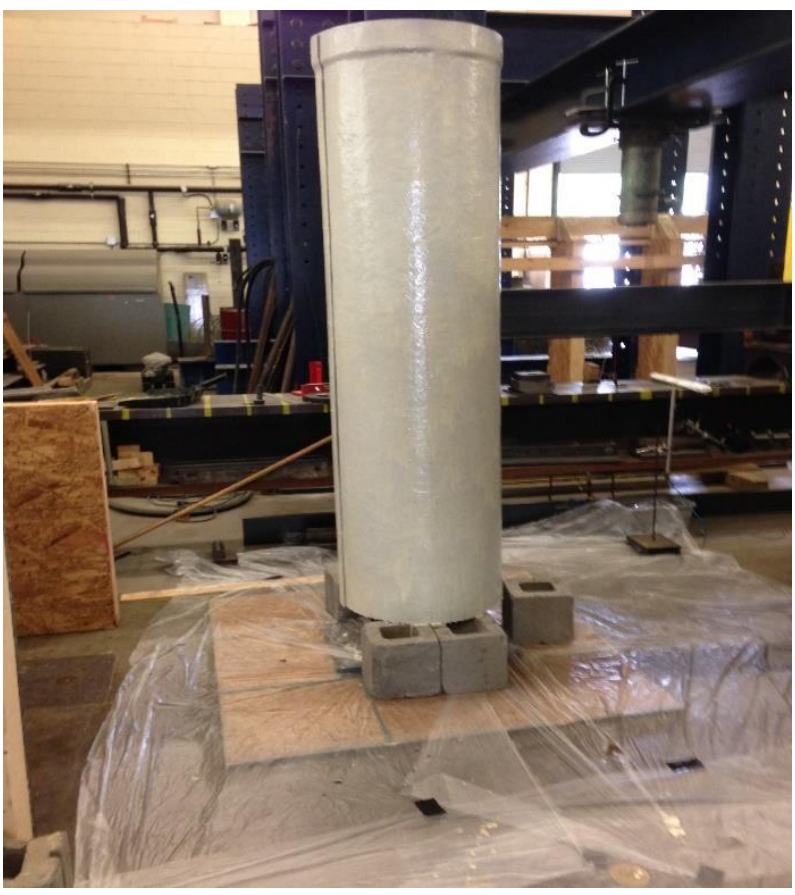

Figure 4-1: Simpsons Strong-TieFx-70 jacket.

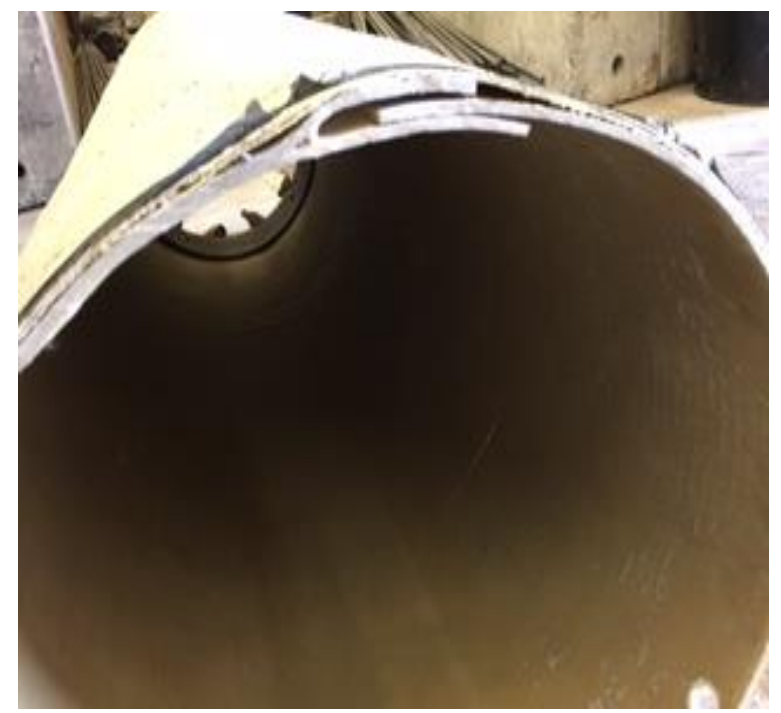

Figure 4-2: Tongue and groove joint. 


\subsubsection{Aquawrap ${ }^{\circledR}$ system}

The thickness of the shell was thin to allow it to be easily installed around the pile, but this limited the strength of the shell. To increase the strength, Aquawrap® FRP wraps were installed on the shell after the auger was attached. Aquawrap ${ }^{\circledR}$ consists of three components available from Air Logistics Corporation.

\section{- Aquawrap ${ }^{\circledR}$ prepreg :}

G-05 Aquawrap ${ }^{\circledR}$ is a bi-directional glass fabric pre-impregnated with water activated urethane resin (Air Logistics Corporation, 2015). Aquawrap® has the ability to cure in wet and underwater conditions with a workable time of 30-40 minutes and curing time of 1 hour. Aquawrap ${ }^{\circledR}$ comes in a sealed protective bag to prevent it from curing in atmospheric moisture and is opened just before application (Air Logistics Corporation, 2015)

Table 4. 2: Properties of Aqua wrap (Air Logistics Corporation, 2015)

\begin{tabular}{|l|l|}
\hline Working time & $30-40$ min. at $77^{0} \mathrm{~F}$ \\
\hline Application Temperatures & $40-200^{\circ} \mathrm{F}$ \\
\hline Cure Time (Dry to touch) & $30-60$ minutes at $77^{0} \mathrm{~F}$ \\
\hline Chemical resistance & $\begin{array}{l}\text { Acetone, mek, toluene, gasoline, ethyl } \\
\text { alcohol and many others }\end{array}$ \\
\hline Full Cure & 2 days at $77^{0} \mathrm{~F}$ \\
\hline
\end{tabular}


Table 4. 3: Technical specifications of Aquawrap Composite (Air Logistics

Corporation, 2015)

\begin{tabular}{|l|l|}
\hline Fabric & G-05 Fabric \\
\hline Reinforcement & Woven Glass, bi-axial Fabric \\
\hline Thickness & 28.5 mils \\
\hline Tensile Strength & 45,000 psi \\
\hline Tensile Load per ply & $12991 \mathrm{bs} /$ in. width \\
\hline
\end{tabular}

\section{- Primer/Adhesive:}

The primer/adhesive is responsible for the bond between FRP wrap and the substrate. BP-4 primer/adhesive is a product of Air Logistics Corporation for use below the water line. It contains Kevlar microfibers to prevent hairline cracking and chipping and is suitable with concrete, steel, wood and fiberglass surfaces. It comes in the form of two components Part A \& Part B that can be mixed by hand or a mechanical mixer (Air Logistics Corporation, 2012).

Table 4. 4: Properties of BP-4 Primer /Adhesive (Air Logistics Corporation, 2012)

\begin{tabular}{|l|l|}
\hline Application Temperature & $55-120^{\circ} \mathrm{F}$ \\
\hline Compressive Strength & $10,000 \mathrm{psi}$ \\
\hline Tensile Strength & $4,800 \mathrm{psi}$ \\
\hline Flexural Strength & $6,600 \mathrm{psi}$ \\
\hline
\end{tabular}




\section{- Stricture banding:}

It is a high strength stretch film as shown Figure 4-3that is positioned tightly across the entire wrapped area. It will compress and consolidate the composite during curing to ensure that a good bond is created between the composite and the underlying surface. It not only contains the composite but also ensures a proper bond with the substrate.

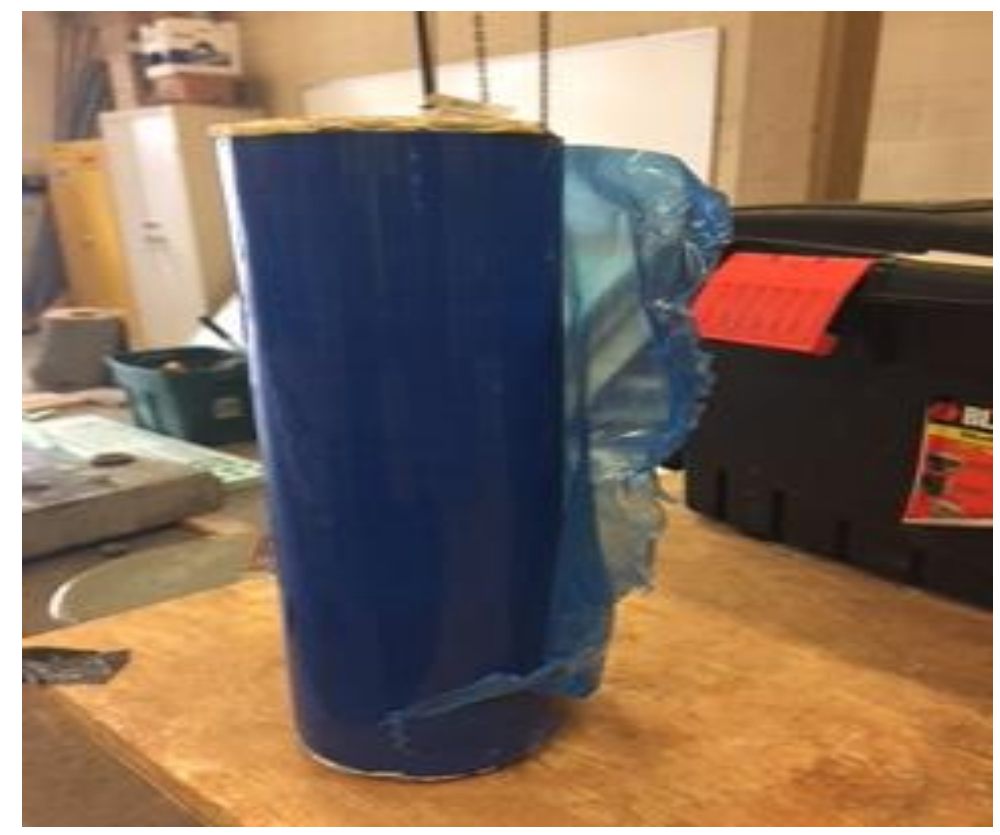

Figure 4-3Stricture Banding. 


\subsection{Lab Testing/Test Setup}

In order to test the installation of the shell/auger system, a 3'X3'X3' box was built and filled with soil. An 8' tall steel wide flange section was placed in the center of the box as shown in Figure 4-4 to replicate an existing pile. The soil is placed in layers and well tamped so that it would be dense up to a depth of 2 feet as shown in Figure 4-5.

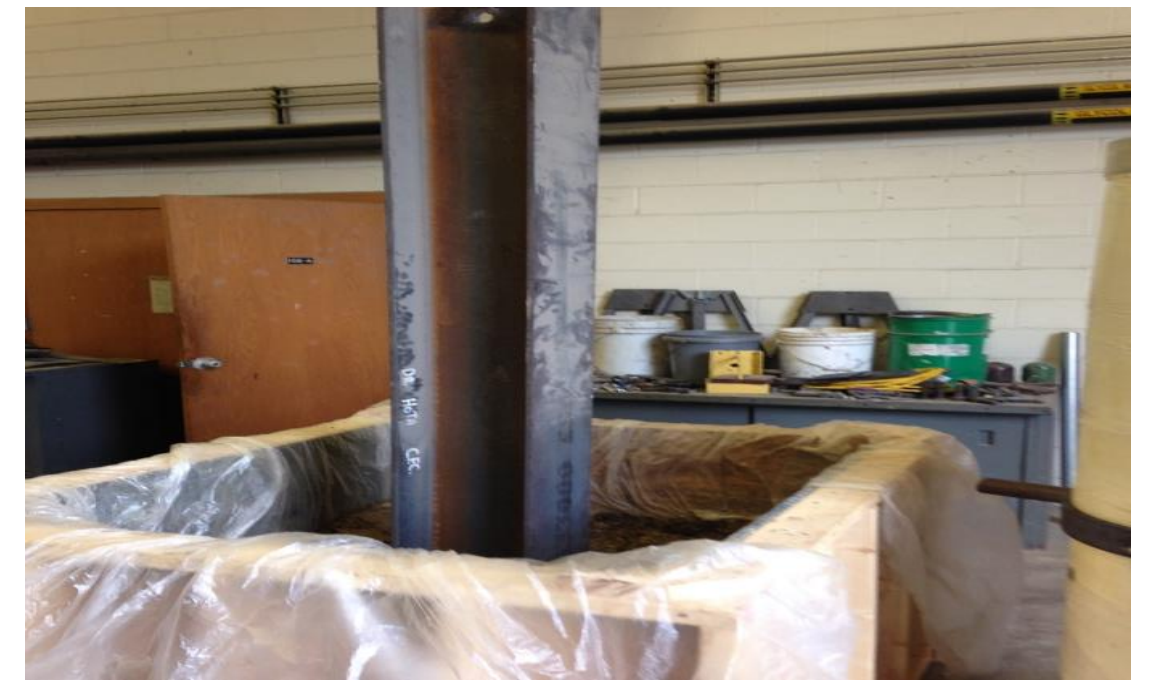

Figure 4-4: Test Box setup with a column.

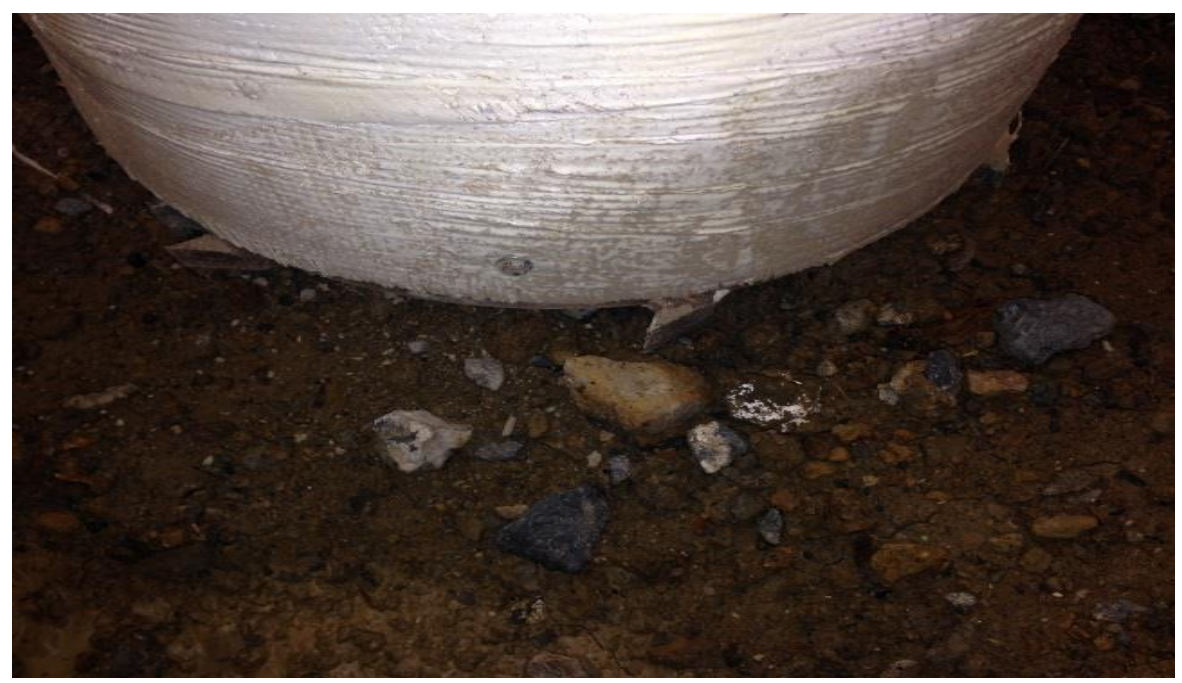

Figure 4-5: Soil rubble. 


\subsubsection{Positioning of Jacket and Auger around the Steel Column}

The open fiber reinforced polymer circular-cylindrical shell/jacket (Figure 4-6) was placed around the column utilizing the longitudinal tongue and groove seam, then the seam was sealed with bolts and epoxy. The auger attachment was then joined to the base of the jacket shell by means of epoxy and bolting at an overlapping portion between the jacket and auger attachment as shown in Figure 4-7.

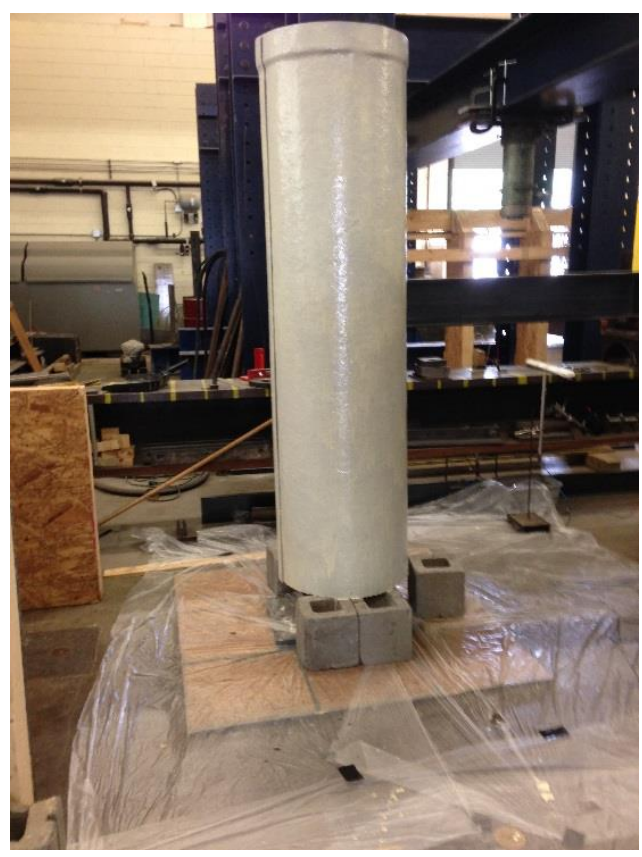

Figure 4-6: FRP Shell. 


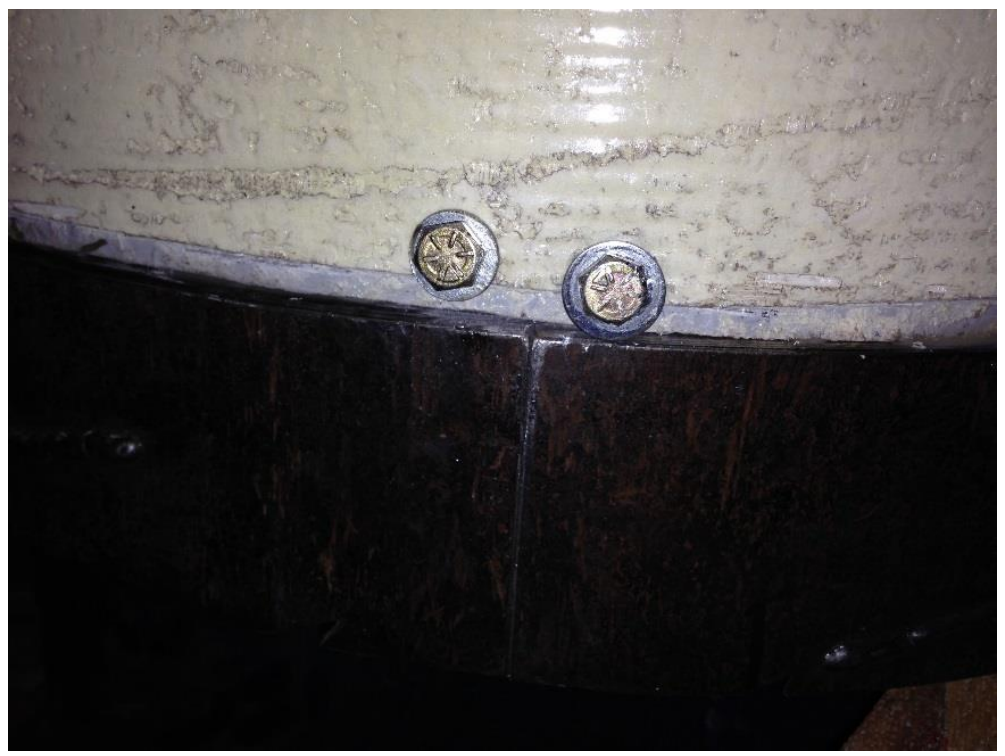

Figure 4-7: Auger adjoined to the FRP shell with bolts and Epoxy.

\subsubsection{Application of Aquawrap®.}

After installing the auger, the BP-4 primer is applied to the FRP shell's outer surface with the help of a spreader. Once the primer becomes tacky, the Aquawrap® rolls are opened and applied. The Aquawrap ${ }^{\circledR}$ is tightly hand-wrapped around the shell by wrapping from the bottom of the shell (Figure 4-8) to make one complete revolution then spirally up the remainder of the shell. Tension is applied to ensure that there are no folds or voids in the wrap (Figure 4-9). Once both layers are applied, stricture banding is tightly wrapped around the Aquawrap ${ }^{\circledR}$. The stricture banding is perforated with the perforator tool (Figure 4-10) so that $\mathrm{CO}_{2}$ gas buildup during the curing process is released. The wrap actually hardens in couple of hours, but it will take around a day to cure completely. Once the wrap completely cures it is ready to be used. 


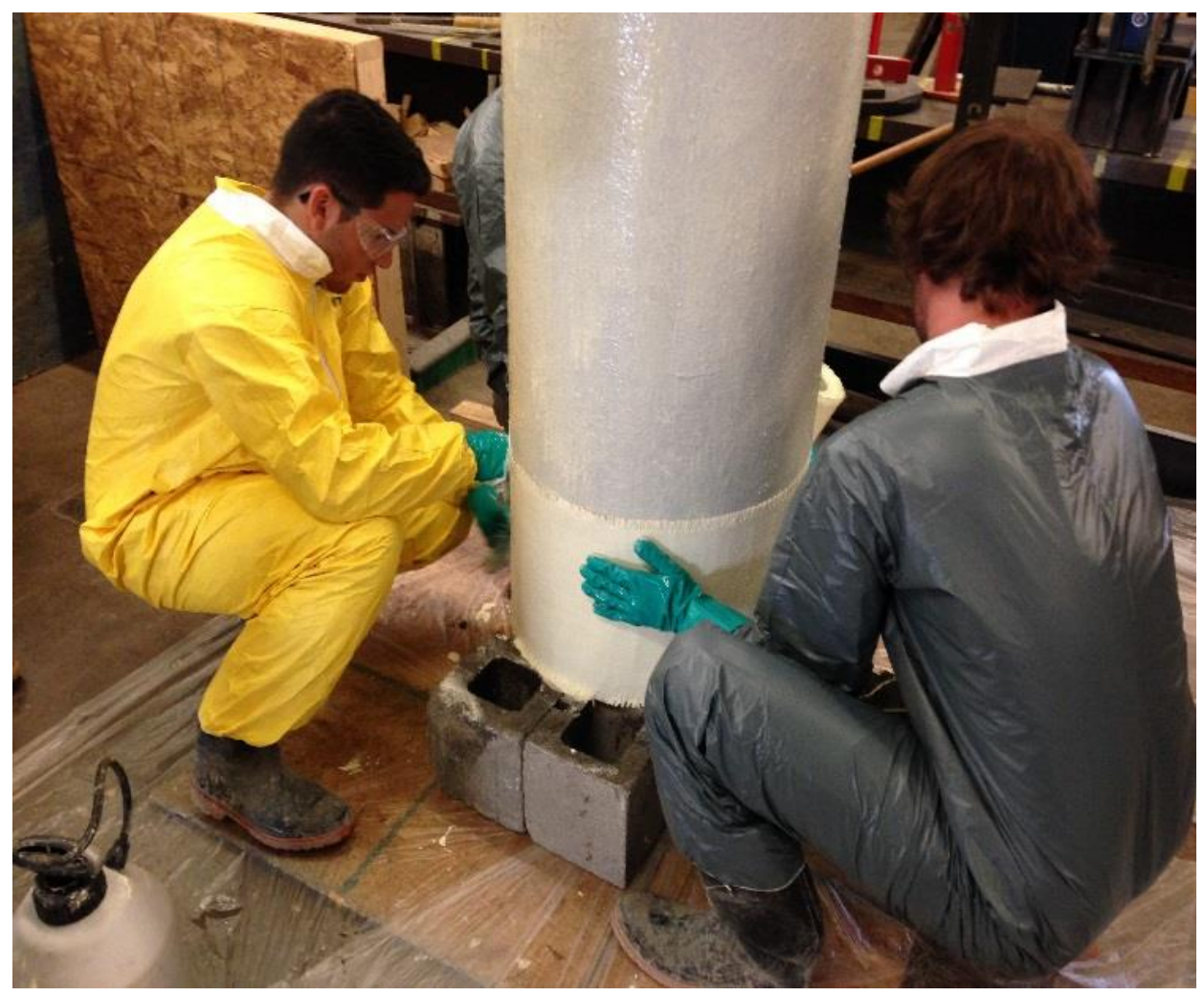

Figure 4-8: Wrapping from the Bottom.

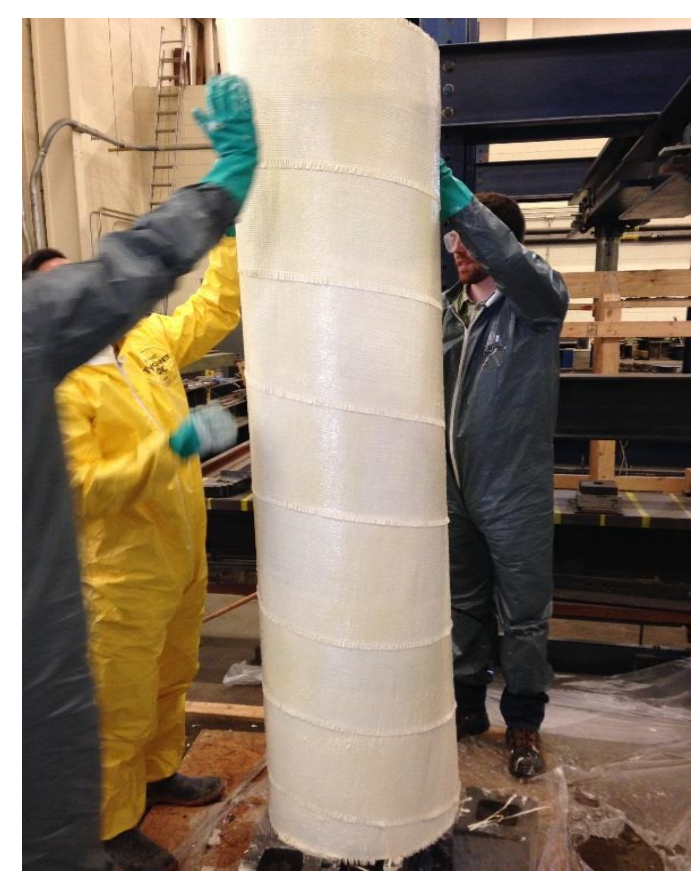

Figure 4-9: Wrapped Shell . 


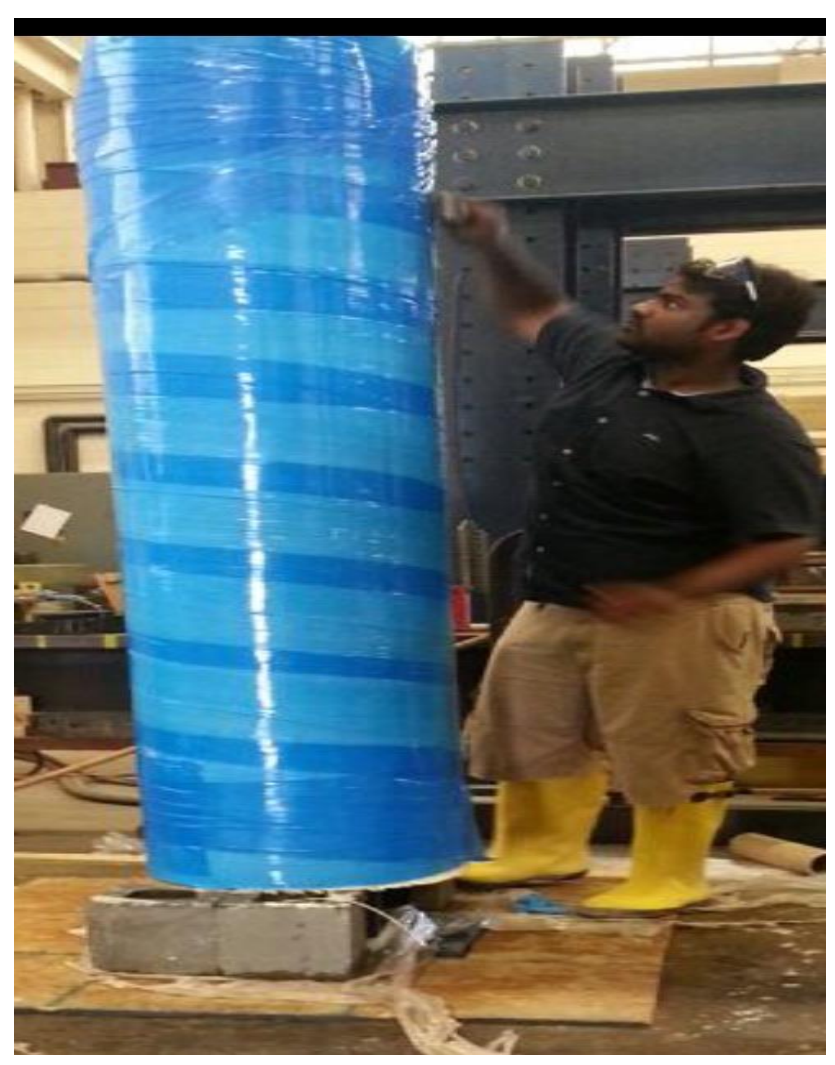

Figure 4-10: Perforation of Stricture banding 


\subsubsection{Driving mechanism:}

For the laboratory testing an adjustable metal split ring as shown in Figure 4-11 was used to provide the torque. The split ring can be positioned at suitable height and can be moved upward as the shell bores into the soil. Two solid cylindrical steel tubes at least 6 ' in length are welded to each of the split ring as shown in Figure 4-12 so that two or more persons can generate torque on the shell as shown in Figure 4-13.

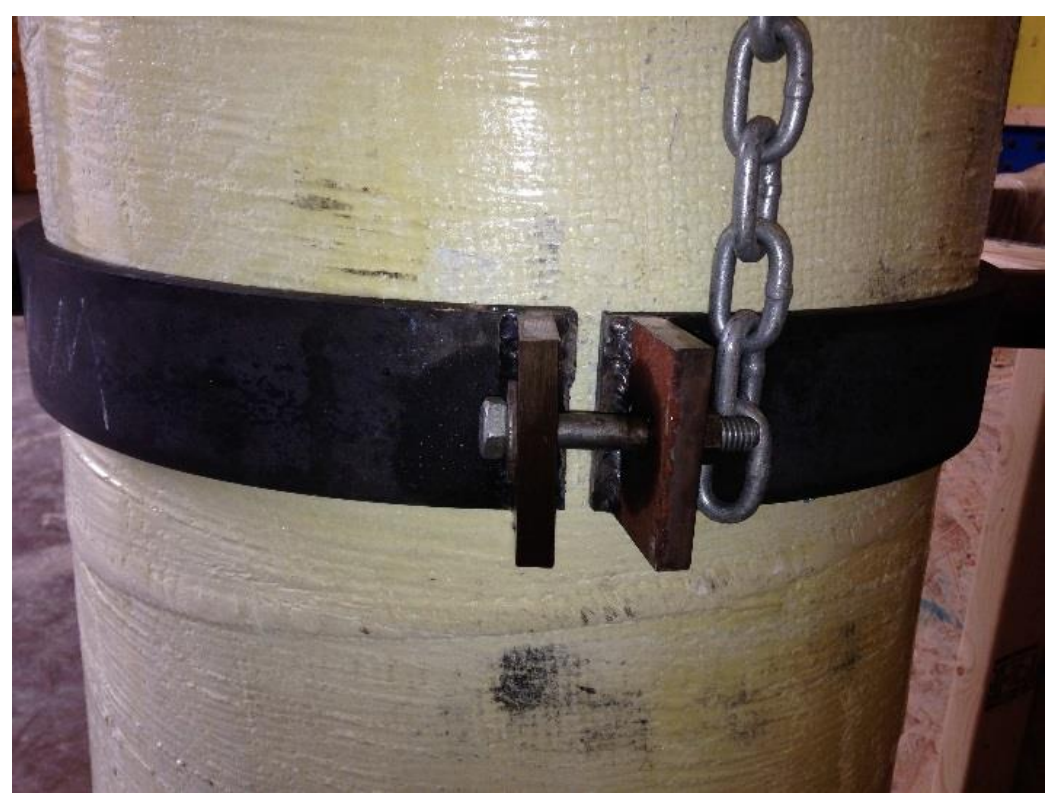

Figure 4-11: Adjustable Split ring. 


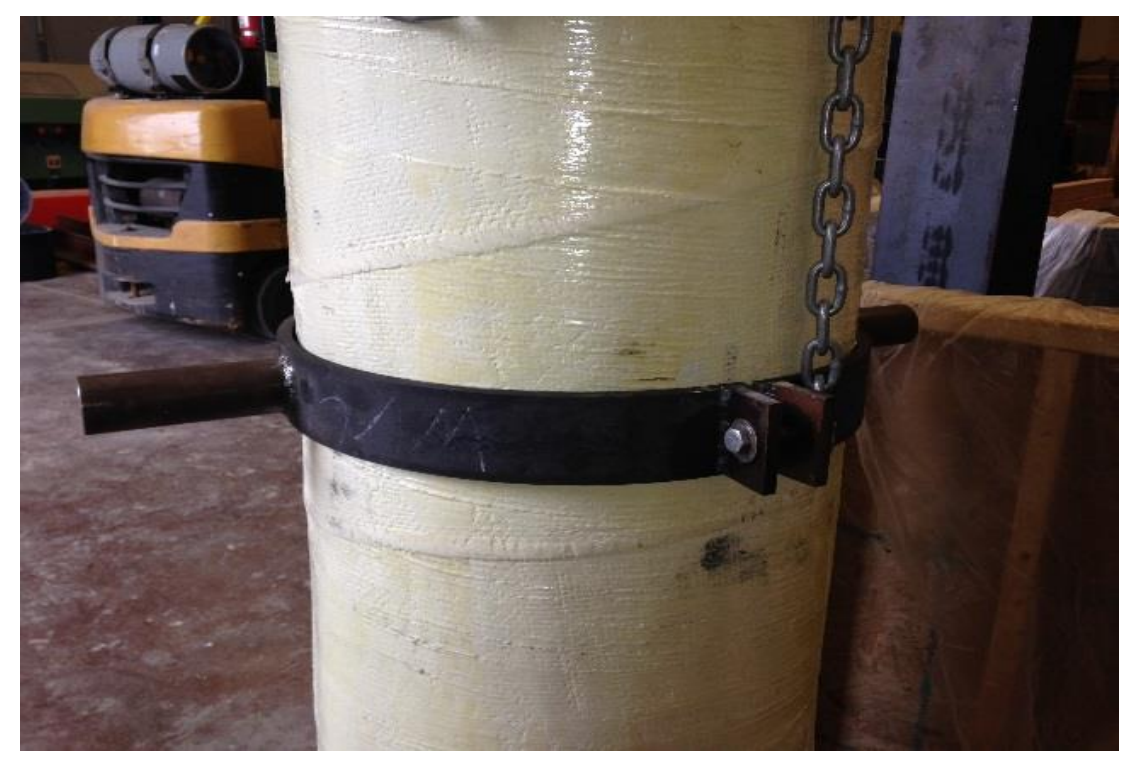

Figure 4-12: Cylindrical attachment to Split ring.

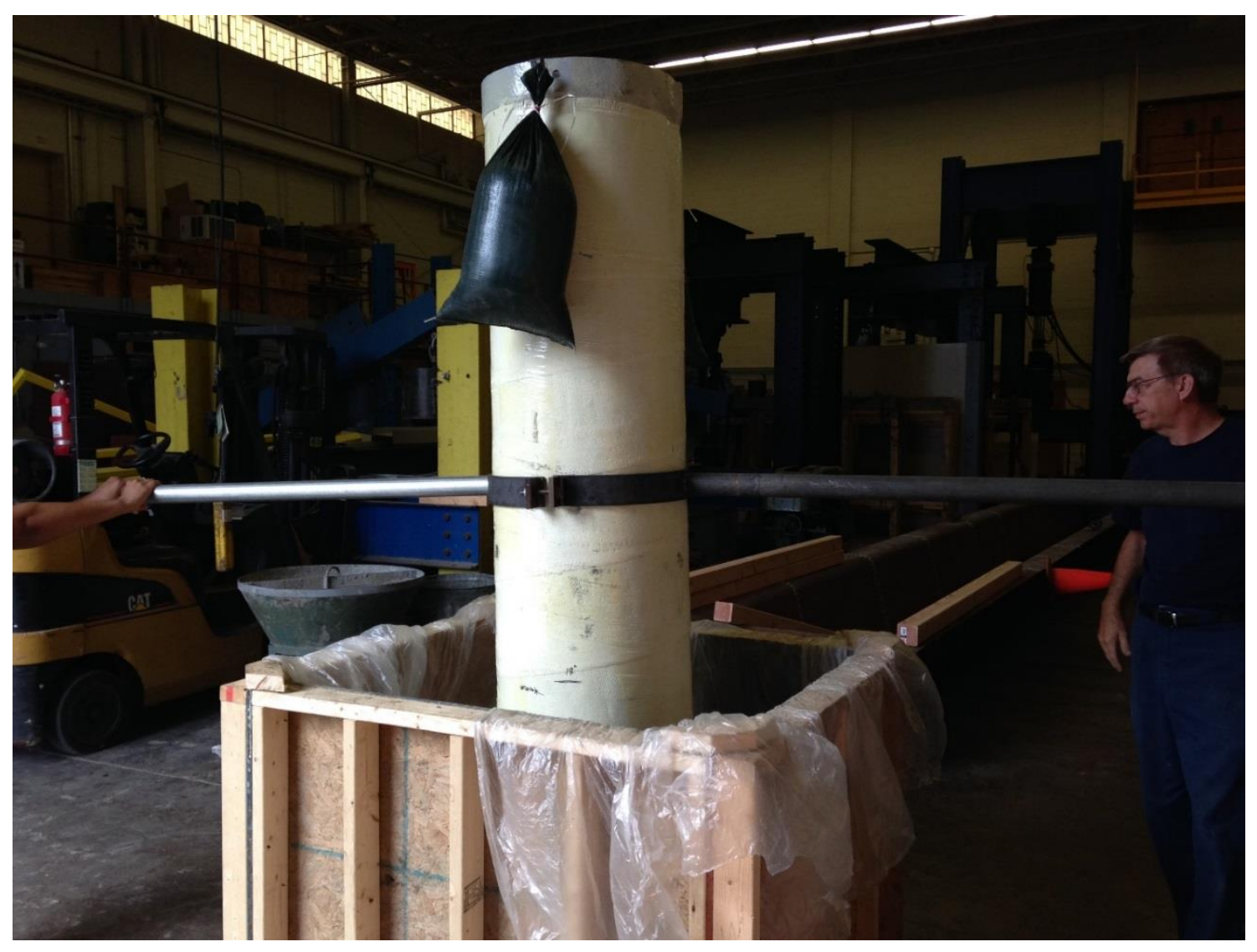

Figure 4-13: Hollow cylindrical tube to produce torque. 


\subsubsection{Testing of the Auger attachments}

Once the fiber reinforced polymer shell with the auger teeth attachment is wrapped with Aquawrap® and cured for 24 hours, it is ready to be tested. Four sand bags each weighing about 501b were hung on the top of the shell as shown in the Figure 4-14 to provide vertical loads. With two people, one on each of the cylindrical tubes, torque is applied by pushing on the tubes. As torque is being applied, the auger attachment drives the shell into the soil as shown in (Figure 4-15). The split metal ring is adjusted to suitable height from time to time as the shell bores down into the soil to maintain a comfortable pushing height. As the auger bores down it pushes the soil aside making way for the shell column (Figure 4-16).

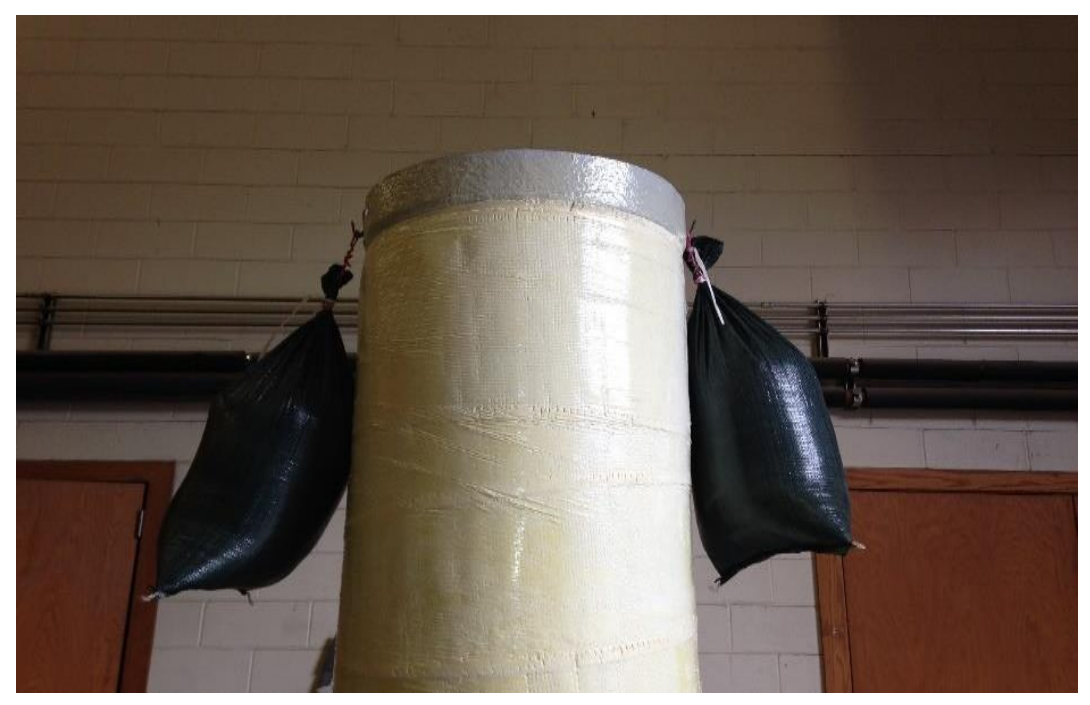

Figure 4-14: Sand Bags. 


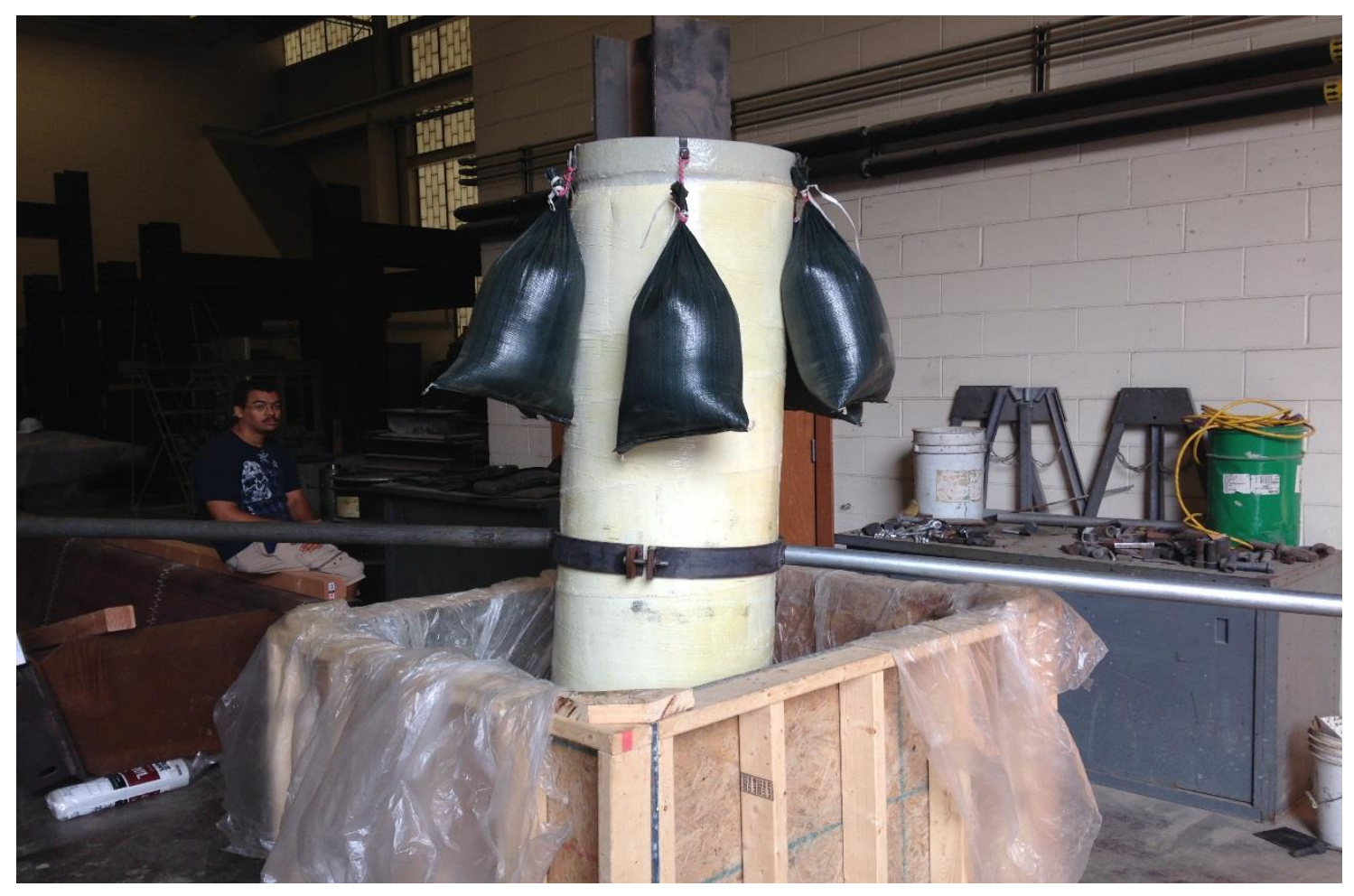

Figure 4-15: FRP shell bored down into the soil.

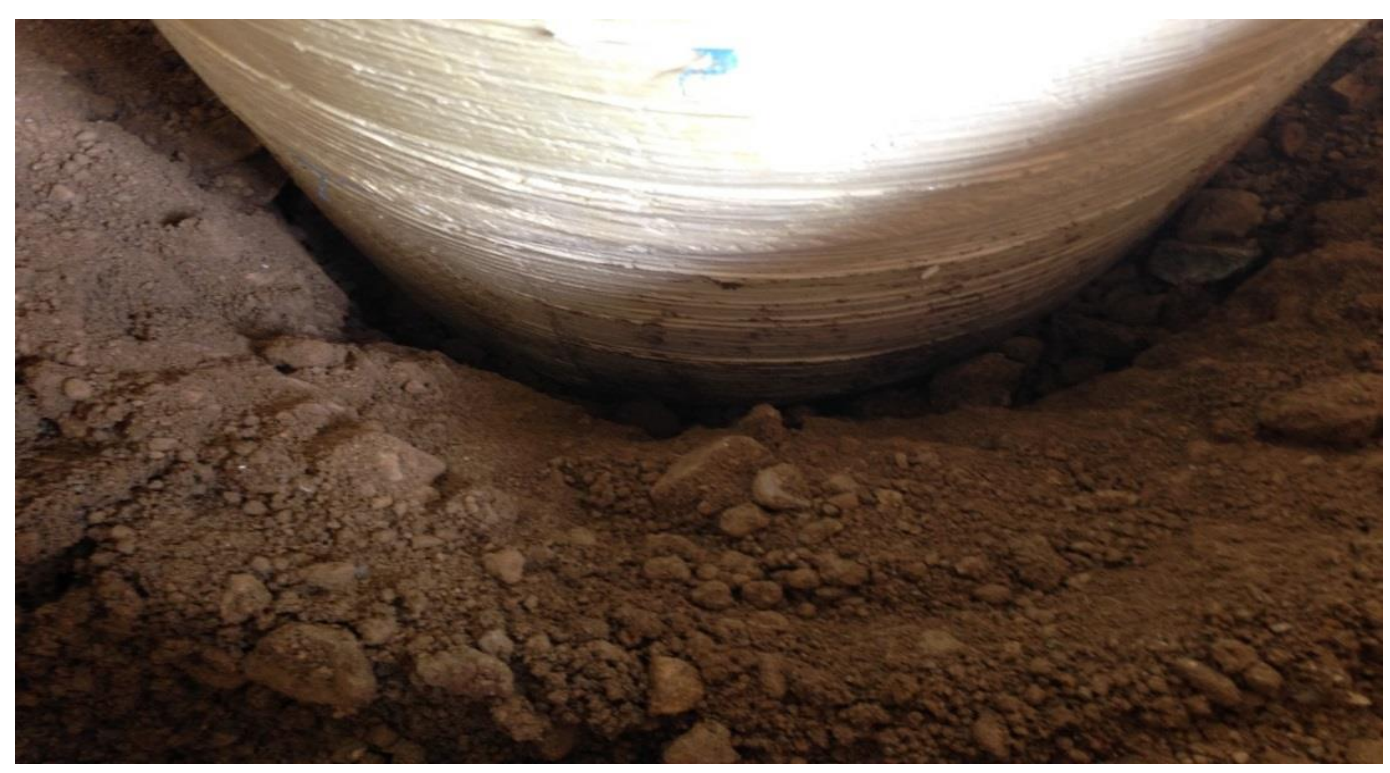

Figure 4-16: The FRP shell going down as soil is pushed aside. 


\subsubsection{Comparison of Auger attachments}

All three auger attachments were tested in the lab test setup. The performance of each attachment is discussed below:

Cutting Edge Auger: This design worked well for 8-10 inches into the soil. Downward thrust is governing force. This auger attachment couldn't push away the pebbles away from the line of driving. This design is best suited for soft soils with no pebbles.

Excavating Teeth Auger: This design drove well for 12-14 inches down into the soil. The specially designed teeth did cut the soil pretty well and made a wider bore hole around the shell reducing the lateral pressure. The additional helical attachments ensured the continuous downward movement of the shell. It did push medium size pebbles but struggled with bigger size ones. It is suitable for soils with small pebbles.

Bullet Teeth Auger: This design worked the best, driving through the 18 inches with ease. It performed well in downward movement and pushing the pebbles away. It is suitable for most soil conditions.

The comparison of all three attachments is tabulated below

\begin{tabular}{|c|c|c|c|}
\hline Auger Attachment & Cost (US Dollars) & Driving depth(inches) & Suitable soil conditions \\
\hline Cutting Edge & $300-400$ & $8-10$ & Soft soils with no pebbles \\
\hline Excavating Teeth & $350-450$ & $12-14$ & Soils with small pebbles \\
\hline Bullet Teeth & $450-500$ & 18 & Almost all soil conditions \\
\hline
\end{tabular}




\subsection{Strain Levels on the shell}

Torque and vertical loads are applied directly on to the fiber reinforced polymer shell system. The hollow shell transfers the torque to the auger attachment thus boring the whole system into the soil. The application of torque can have varied effects on the shell. It might rupture the surface or sometimes even crush the shell if torque is not applied properly. Thus, there is a need to monitor the stresses in the FRP shell

There are two major locations needed to check the strains or stresses, i.e. where we apply the torque to rotate the auger and at the soil and FRP interaction. The stresses at the point of application of torque depend on the mechanism of application of torque. The stresses at the point of interaction between the soil and FRP shell are of major concern due to the lateral pressure applied by the soil on to the shell and frictional force between the soil particles and outer surface of the shell.

Strain gages are placed at heights of 36 " and 14" from bottom of the auger attachment, both in longitudinal direction and circumferential directions. The 36" corresponds to the torque applied and 14" corresponds to the soil interaction. The strain readings from the four strain gages obtained from the data acquisition system while the shell was driven into the ground are shown in Figure 4-17 


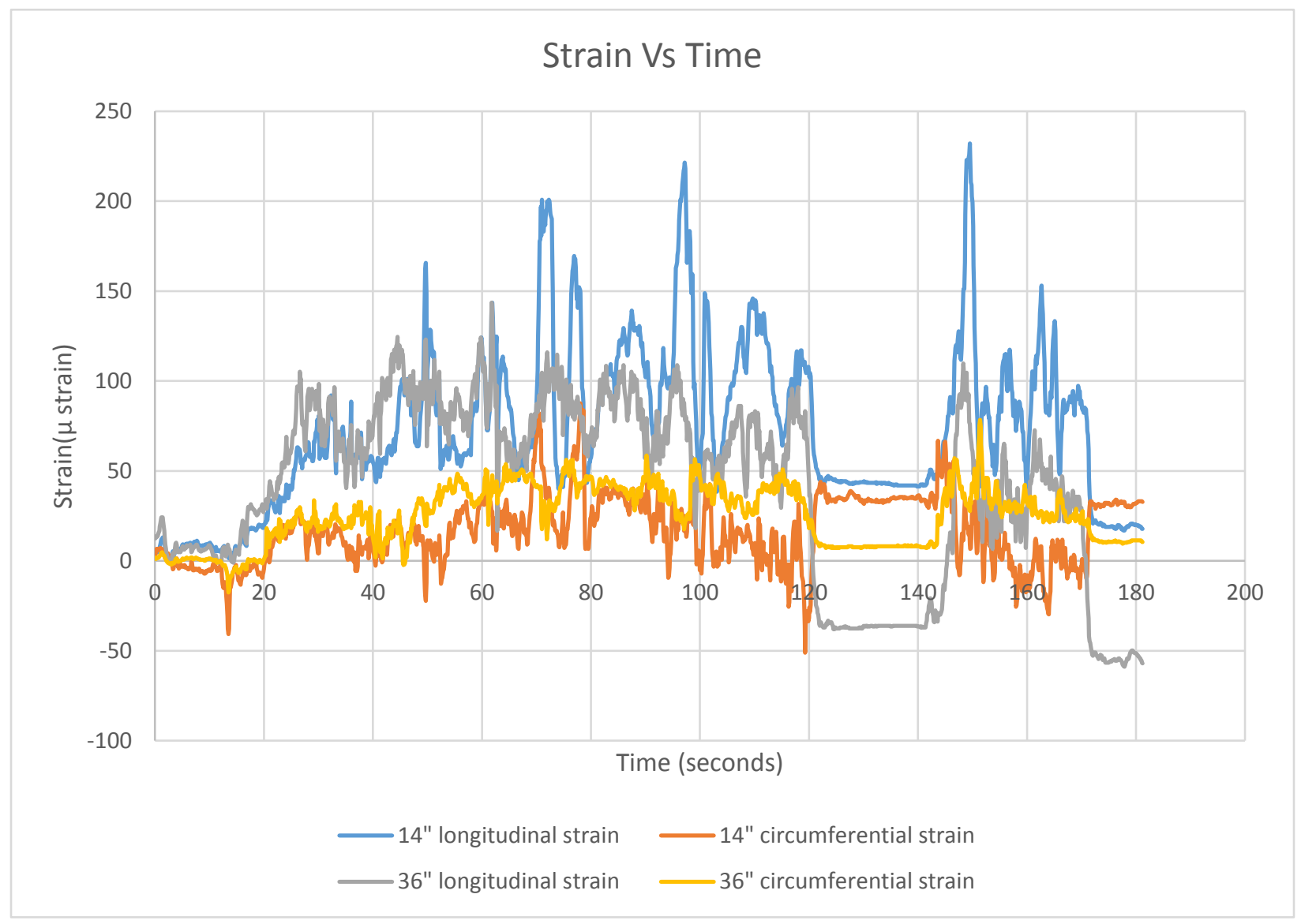

Figure 4-17: Figure Strain Vs Time Graph of all the four Strain gages.

The test was carried out over 3 minutes (180 seconds) and the strain gage readings were plotted on the Y-axis against time on $\mathrm{X}$-axis. The longitudinal strain gages placed at 14" read higher strains due to the interaction with the soil, but the circumferential strains are fairly close. The steady region between the 120 seconds and 140 seconds on the graph is due to a pause in application of torque. 
The highest values of strain from each strain gage are noted down. Corresponding stress is calculated by using the modular ratio to take into effect the different material properties of the wrap and shell. The calculation of stress is shown in detail in Appendix A. The highest strains and corresponding stresses are tabulated below.

\begin{tabular}{|c|c|c|c|}
\hline Location & Direction & Strain( $\boldsymbol{\mu}$ strain) & Stress (psi) \\
\hline 14 inch & Longitudinal & 232.1 & 204.5807271 \\
$\mathbf{1 4}$ inch & Circumferential & 87.6 & 76.71777266 \\
36 inch & Longitudinal & 143.6 & 126.6284156 \\
36 inch & Circumferential & 78.4 & 69.13417674 \\
\hline
\end{tabular}

The maximum stress at each location when compared to the failure stress of the wrap system tend to show that they are far less. The maximum stresses the wrap system can take is about 47000 psi (Soti, 2014) for a dual layer Aquawrap®. 


\subsection{Results}

Based on the test of the three designed auger attachments in terms of simulated field conditions, as created in the lab, following conclusions can be made:

- The FRP jacket - auger system with the tapered cutting edge did drive smoothly into the soil for the first 8-10 inches with the manual application of torque.

- The FRP jacket-auger system with the cutting teeth and screw attachments performed much better than the tapered edge alone and was effective in pushing away the mid-sized rocks, thus clearing the path for the FRP jacket.

- The FRP jacket-auger system with the bullet teeth was the most effective of all. It was able to drive through a depth of 18 inches with ease compared to the other designed models. These bullet teeth can push away big pebbles too.

- Maximum strain of 232.1 micro strains along the longitudinal direction at 14 " from the bottom of the shell and corresponding stress value is $204.58 \mathrm{psi}$.

- Maximum strain of 143.6 micro strains along the longitudinal direction at 36 " from the bottom of the shell and corresponding stress value is 126.62 psi.

- The stress values are very low compared to the failure stress of $47000 \mathrm{psi}$. 


\section{Chapter 5. Compounding Machine}

\subsection{Introduction}

Most plastics are a non-biodegradable and take some thousands of years to disintegrate in

to environment. Plastic compounding is one recycling methods of plastic waste which adds structural stability and does not release trapped carbon into atmosphere. Additives play a major role in compounding as they modify the properties of plastics. Most of the modern compounding machines are rooted to $19^{\text {th }}$ century rubber industry (Zehev Tadmor, 2006). This chapter discusses in detail about the fabrication of a rotatory compounding machine for comingled plastics and how the compounding process is done through recycling.

\subsection{Compounding Machine}

There are many kinds of compounding machines including rotary, single screw melt extruder, co-rotating disk plasticizing processor, twin screw extruder, counter rotating twin screw machines etc. Every machine has the same basic principle of liquefaction of raw materials to have good mixing and bonding of plastics and additives. Each machine has a unique way in which they achieve the liquefaction of plastics. In a rotary compounding machine, the raw materials are ground at very high speeds in a closed chamber with grinding block on a rotating shaft.

This process of compounding starts from feeding of raw materials into the machine. Shredded plastics along with desired additives are the raw materials used. Once shredded raw materials are fed, they must be contained in a closed chamber where they can be grounded and melted by grinding blocks mounted on a shaft rotating at high speeds and should have an 
outlet/dumping provision to collect the compounded plastics. The high speed rotation of shaft and friction between the raw materials and grinding blocks produce heat that needs to be cooled down. A coolant (water) can be used to control the temperature produced in the closed chamber.

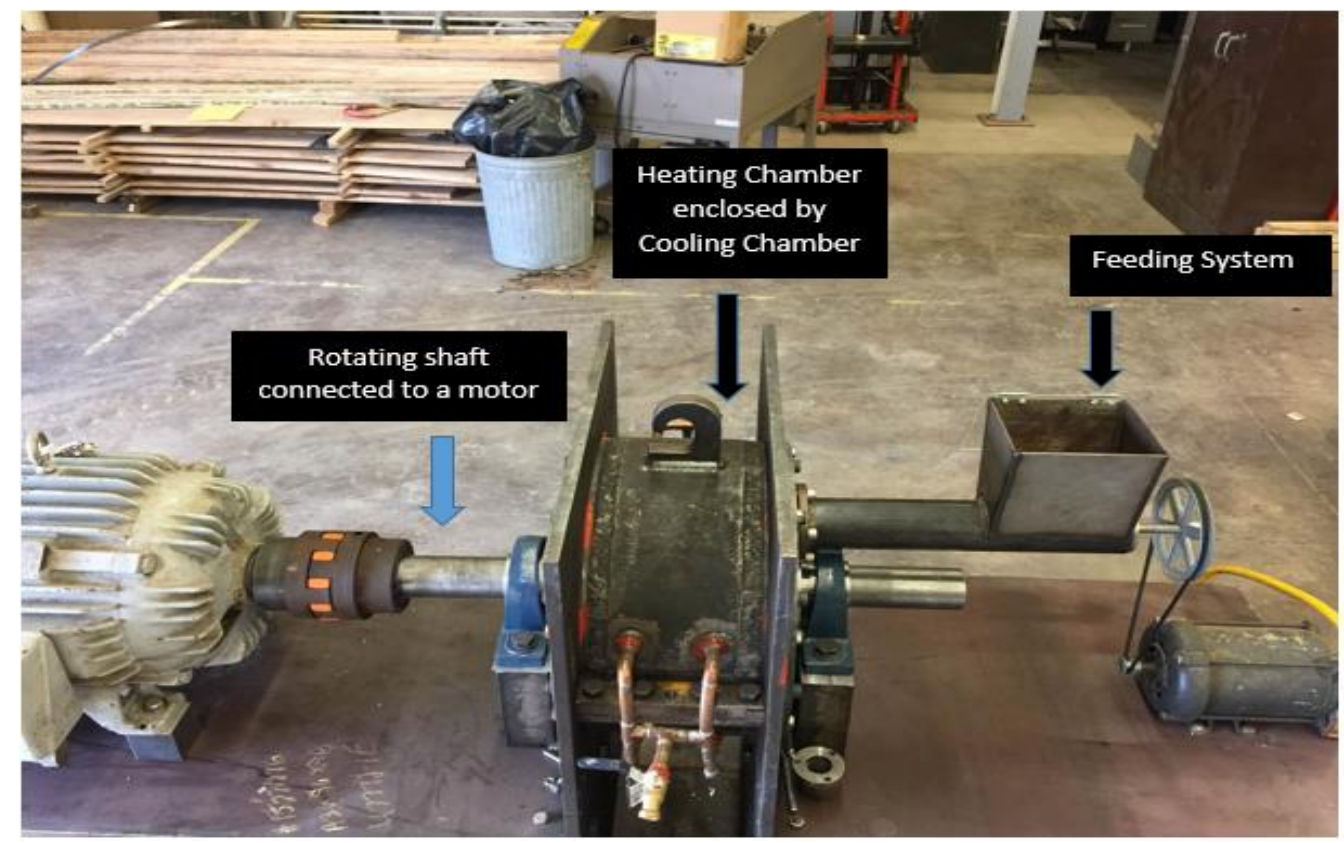

Figure 5-1: Schematic diagram

The major components of the compounding machine are:

- Feeding System

- Heating chamber

- Cooling Chamber 


\subsubsection{Feeding System}

The feeding system consists of feeding hopper (Figure 5-2) and a screw auger (Figure 5-3). The feeding hopper is a converging trapezoidal prism structure with open top through which the material can be fed. The bottom of the feeding hopper opens into a tube containing the screw auger. The screw auger consists of a spiral blade coiled around a shaft driven by a motor on one end and held at the other end to the circular plate of heating chamber. The tube is fastened to the circular plate with bolts, and the circular plate has an opening exactly equal to the diameter of the tube (Figure 5-4, Figure 5-5) to feed the material from the tube to the heating chamber.

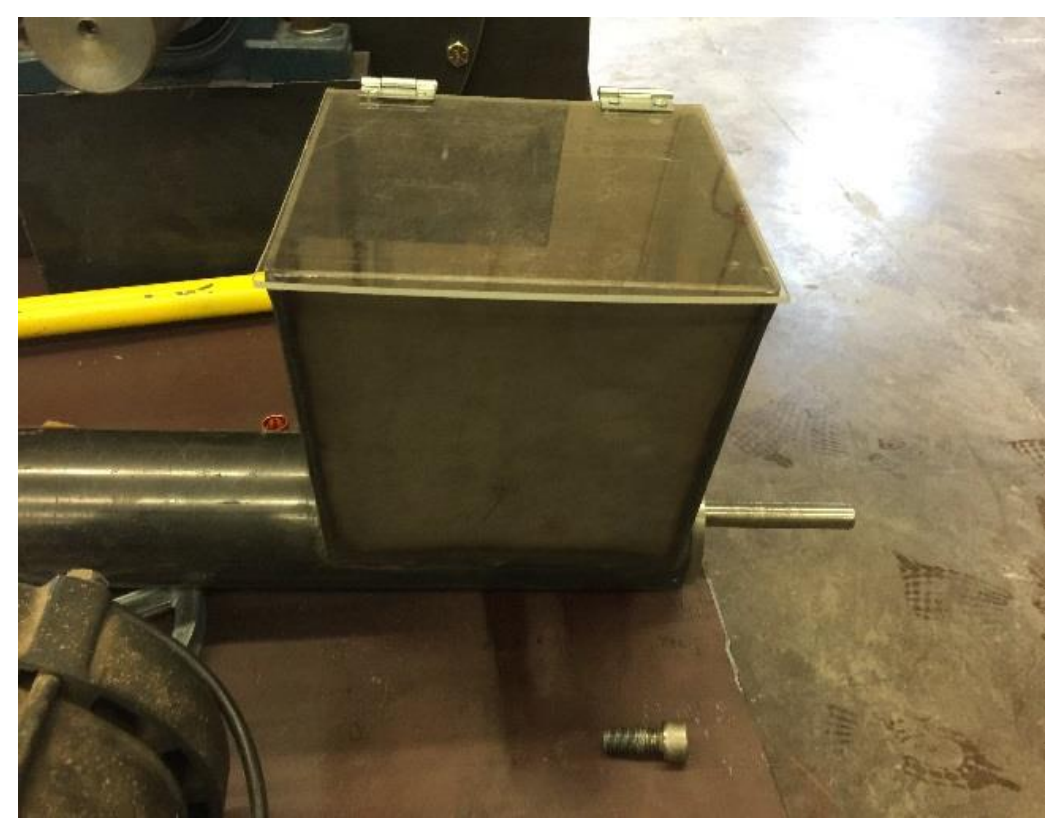

Figure 5-2: Feeding Hooper. 


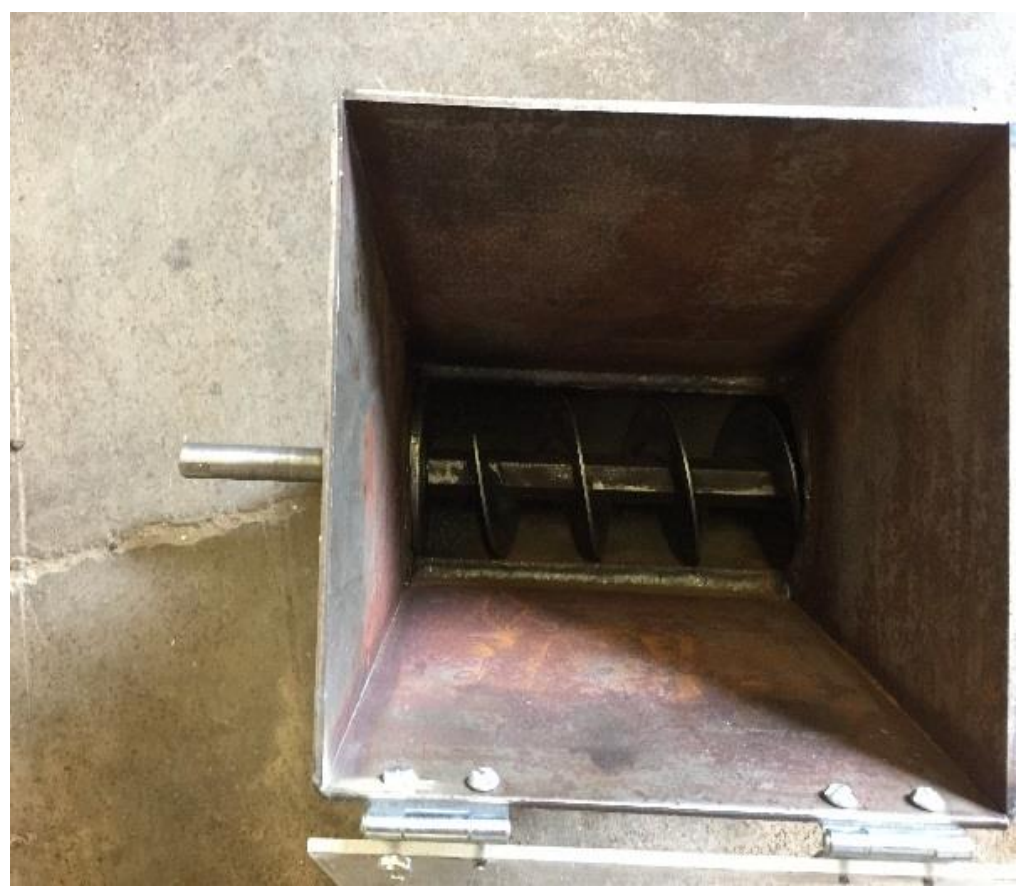

Figure 5-3: Screw Auger.

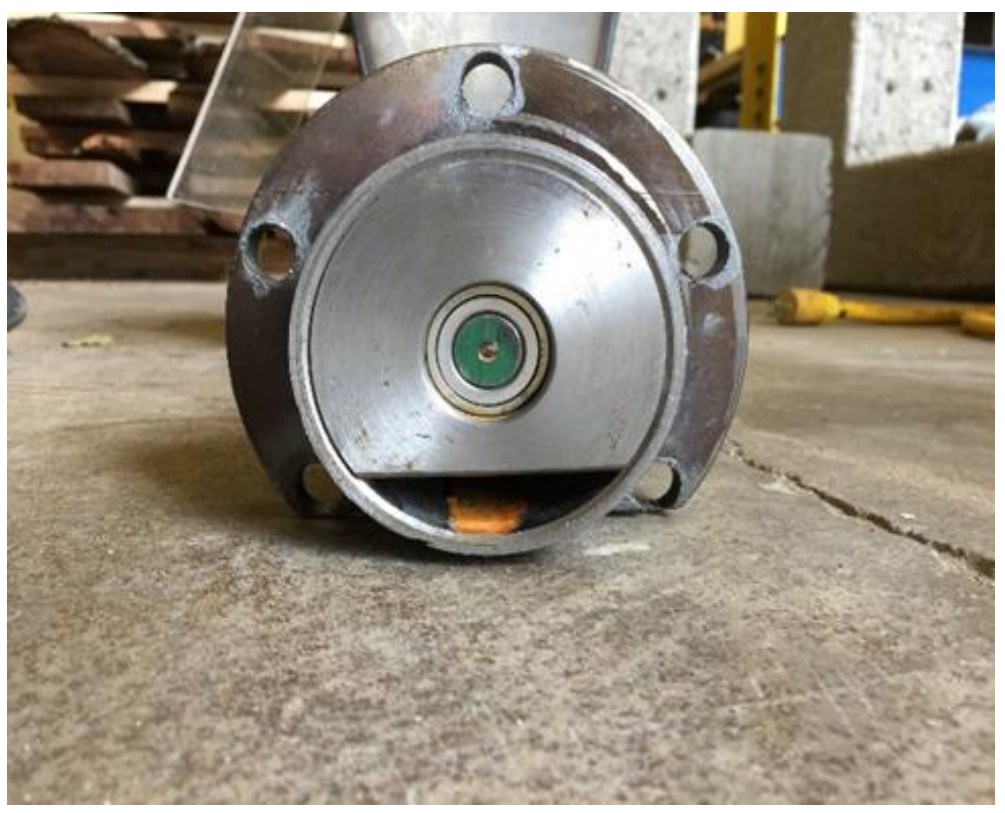

Figure 5-4: Opening on one end of the Screw Auger. 


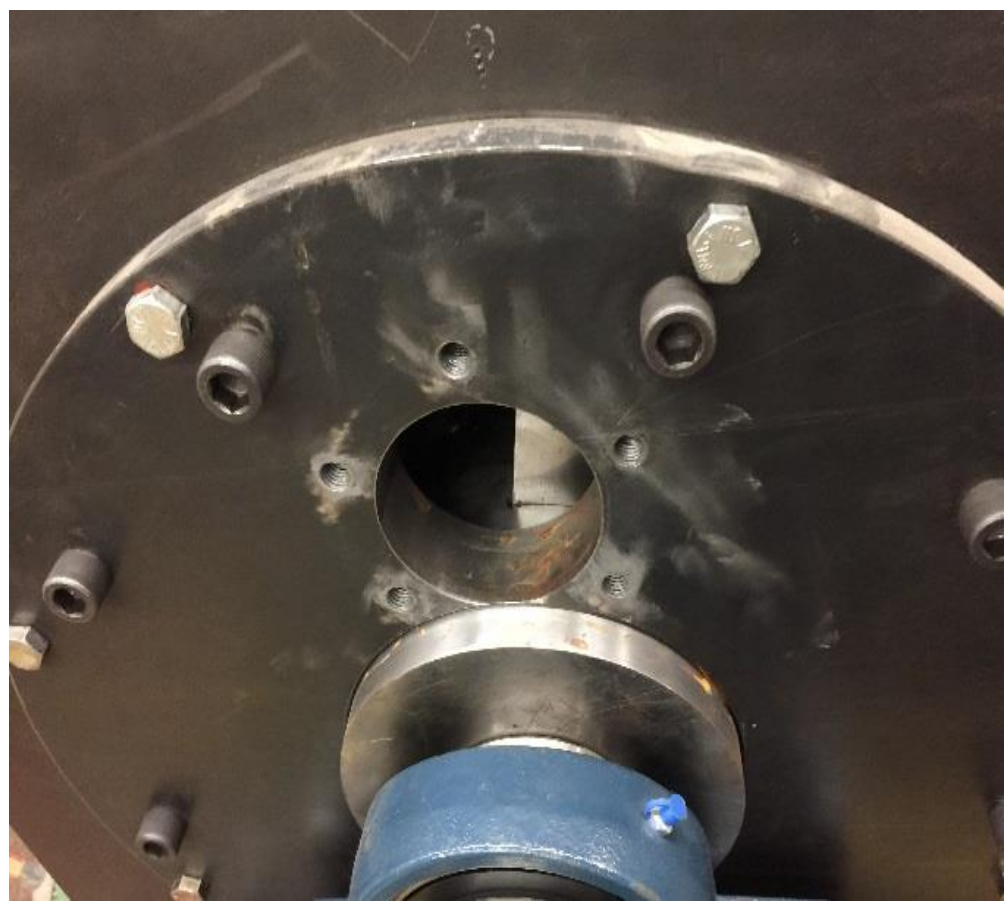

Figure 5-5: provision on the plate to hold the tube.

The shaft of the screw auger rests on two bearings on both ends of the tube. The diameter of bearings is same as the inner diameter of the tube. The spiral blade on the shaft has a diameter less than the inner diameter of the tube to have smooth transition of materials. The bearing at the entrance of the heating chamber has a small opening (Figure 5-5) to have controlled feed of material into the heating chamber while the other bearing is completely closed. The length of the shaft is greater than the length of the tube, thus extending outside of the closed bearing (Figure 5-6). 
The screw auger shaft is run by a motor with the help of two pulleys; one on the auger shaft (Figure 5-7) and one on the motor shaft connected by looped belt. The pulleys reduce the rotation from the $1800 \mathrm{rpm}$ motor to $300 \mathrm{rpm}$.

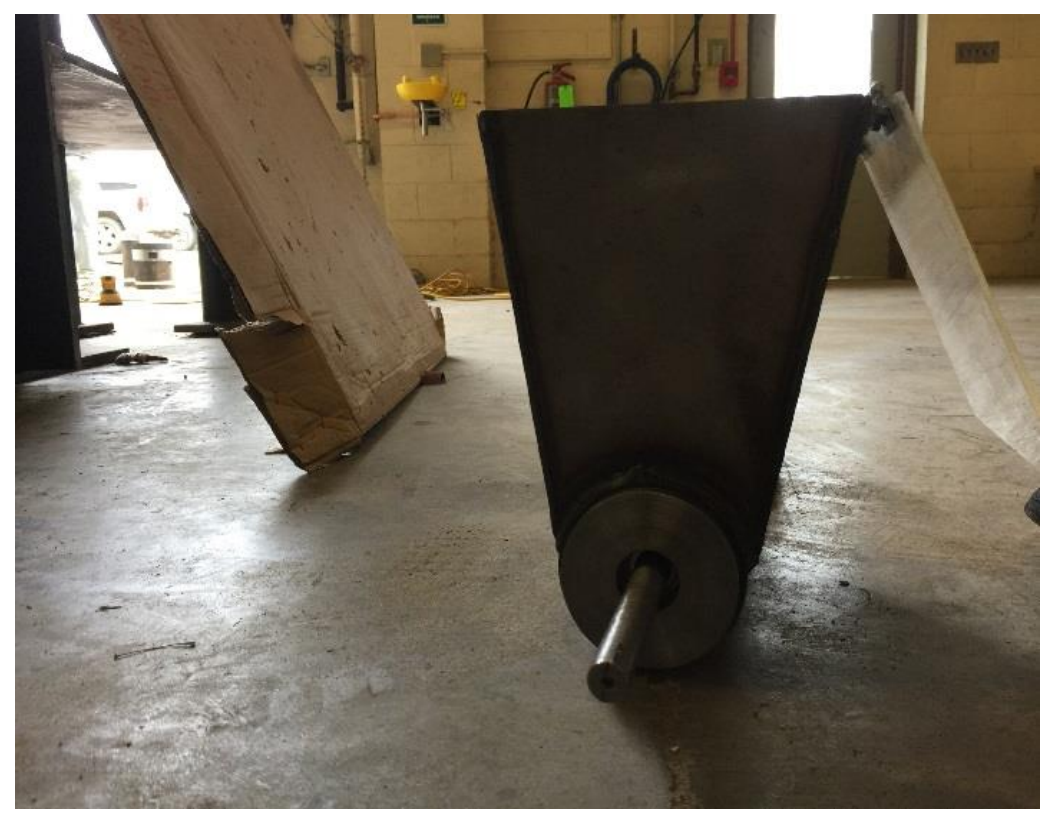

Figure 5-6: Screw Auger Shaft.

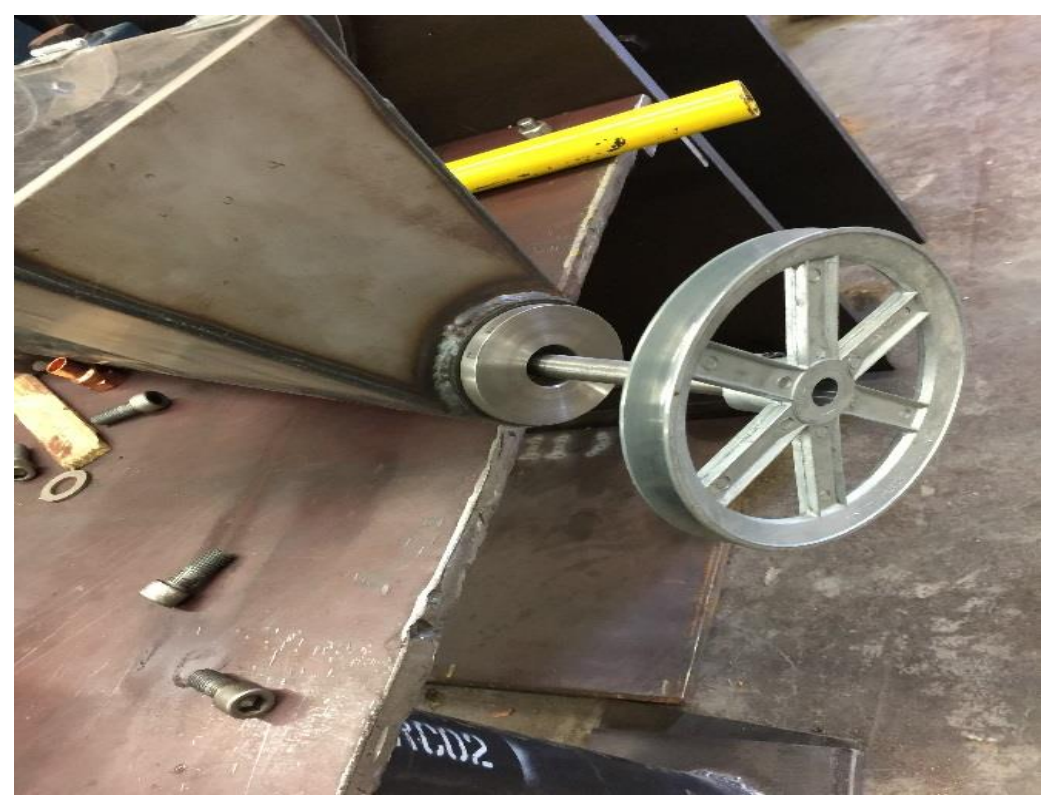

Figure 5-7: Pulley on the shaft. 


\subsubsection{Heating chamber:}

The heating chamber is the primary component of the compounding machine. It consists of a cylindrical chamber (Figure 5-8) enclosed by circular plates (Figure 5-10) on both sides. The heating chamber is designed to position the grinding teeth attached on the circular shaft (Figure 5-11) running across it exactly at its center.

The cylindrical heating chamber is made with high strength rolled steel semicircular components that are bolted and welded together (Figure 5-12) to form a seamless chamber. On one of the semicircular components there is an opening for the dump door so that the compounded plastic can be removed (Figure 5-9). The dumping arm is similar component of the heating chamber made to the exact dimensions of the void provided in one of the semicircular components of the chamber.

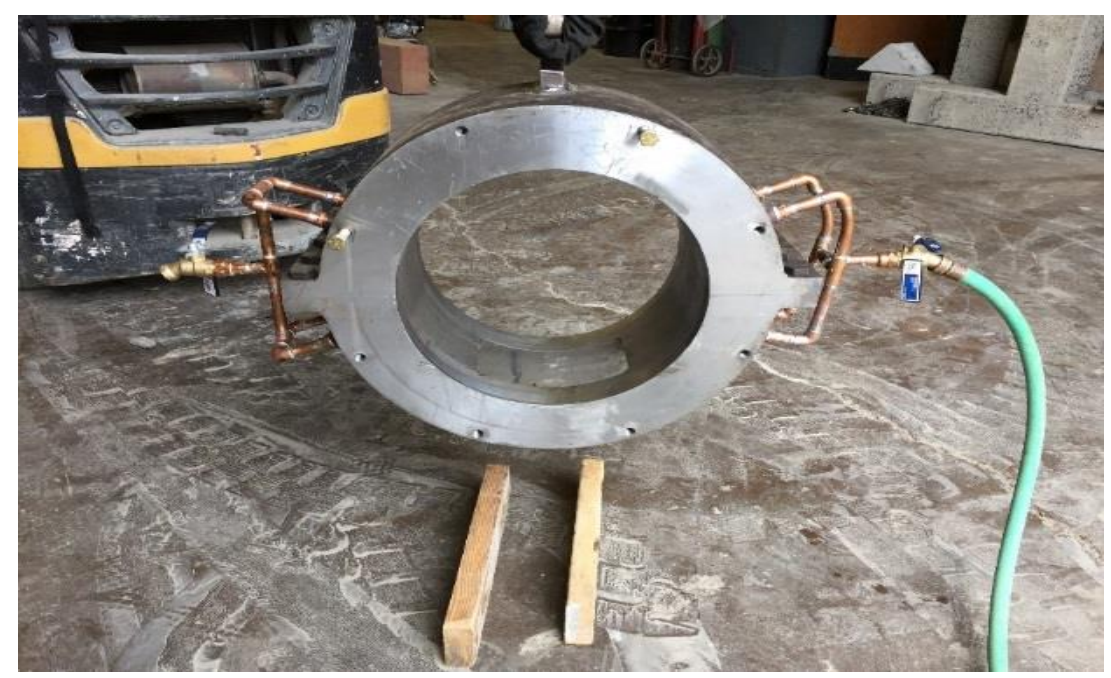

Figure 5-8: Cylindrical chamber. 


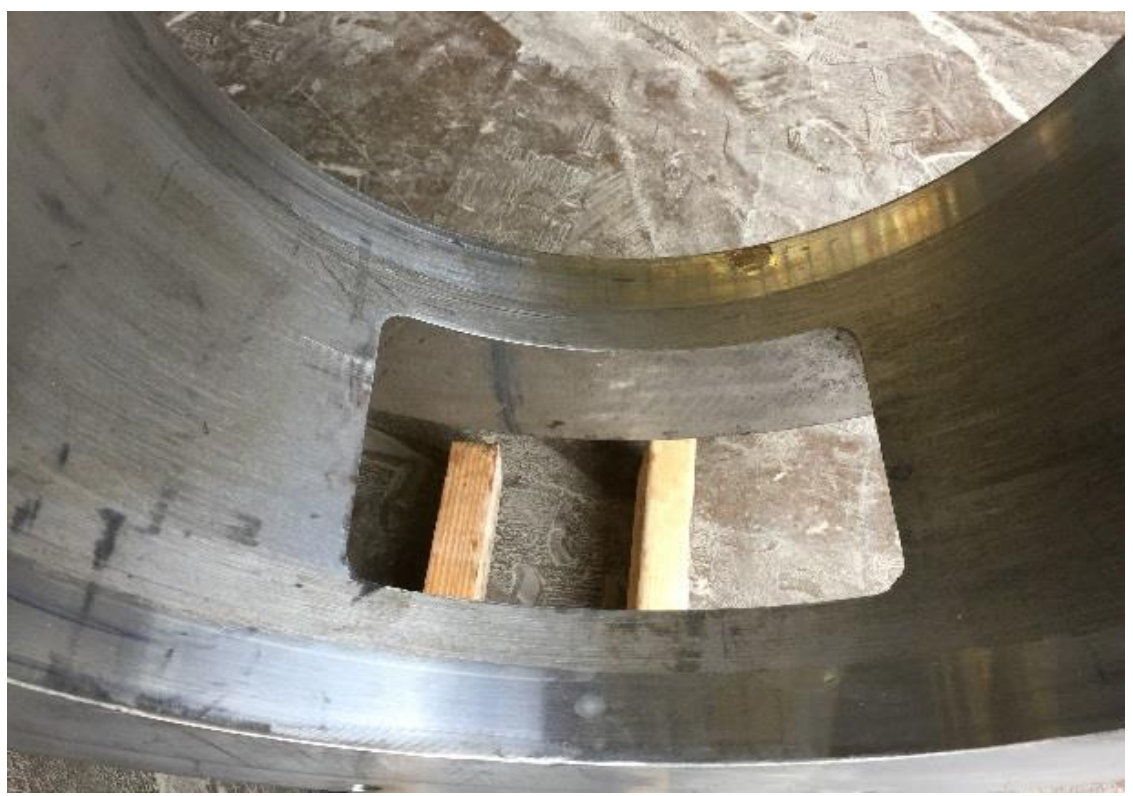

Figure 5-9: Opening for dump door.

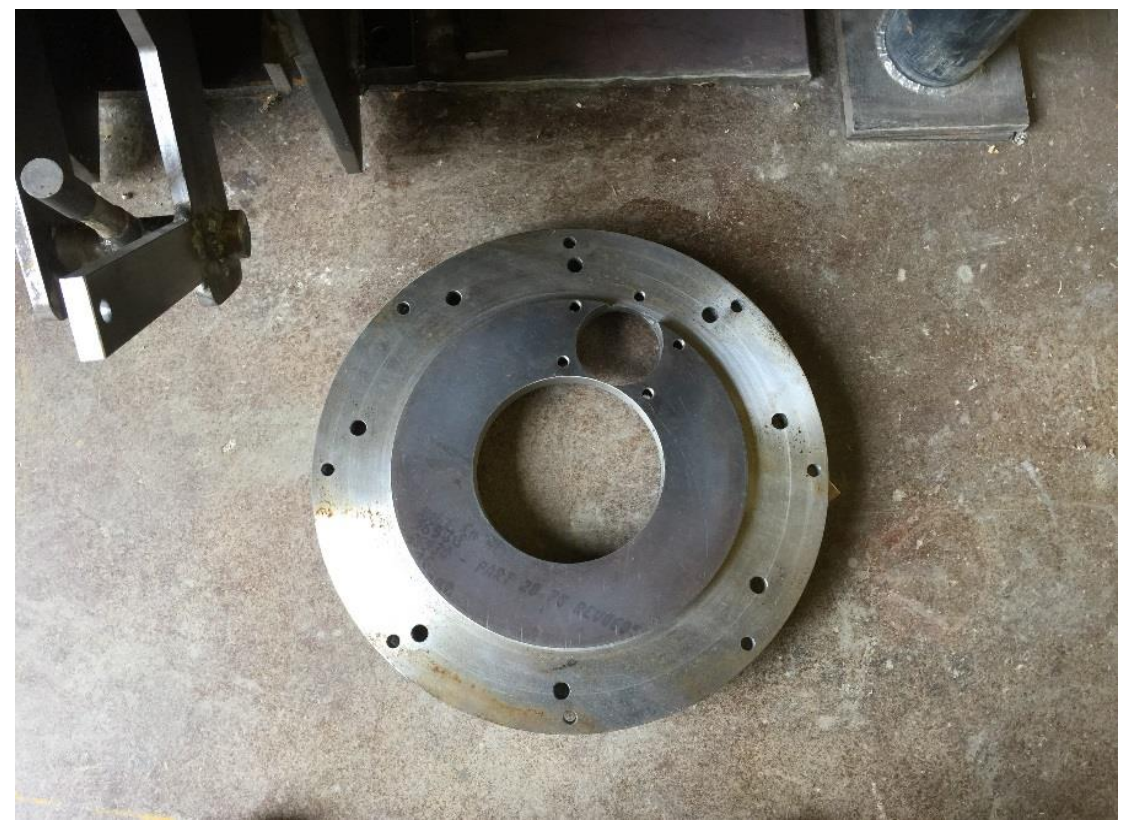

Figure 5-10: Circular plate of the cylindrical chamber. 


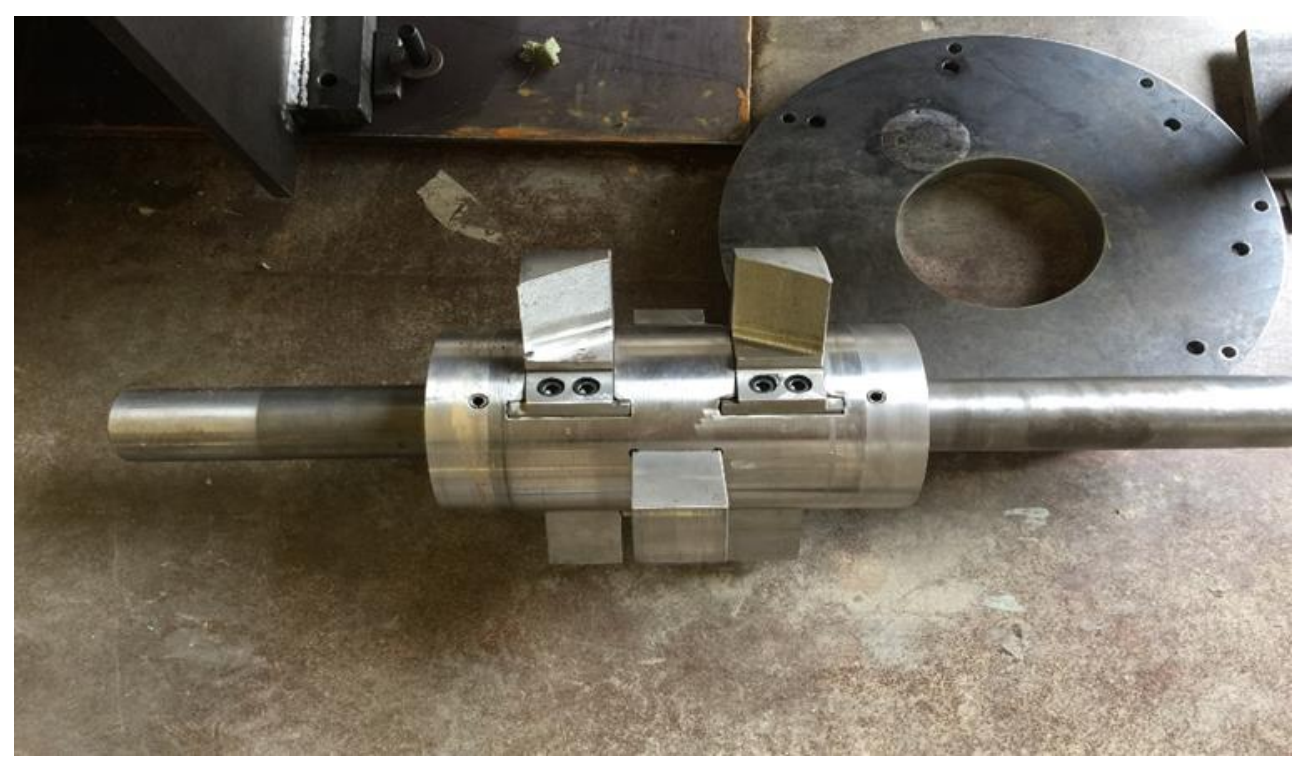

Figure 5-11: Shaft with grinding teeth.

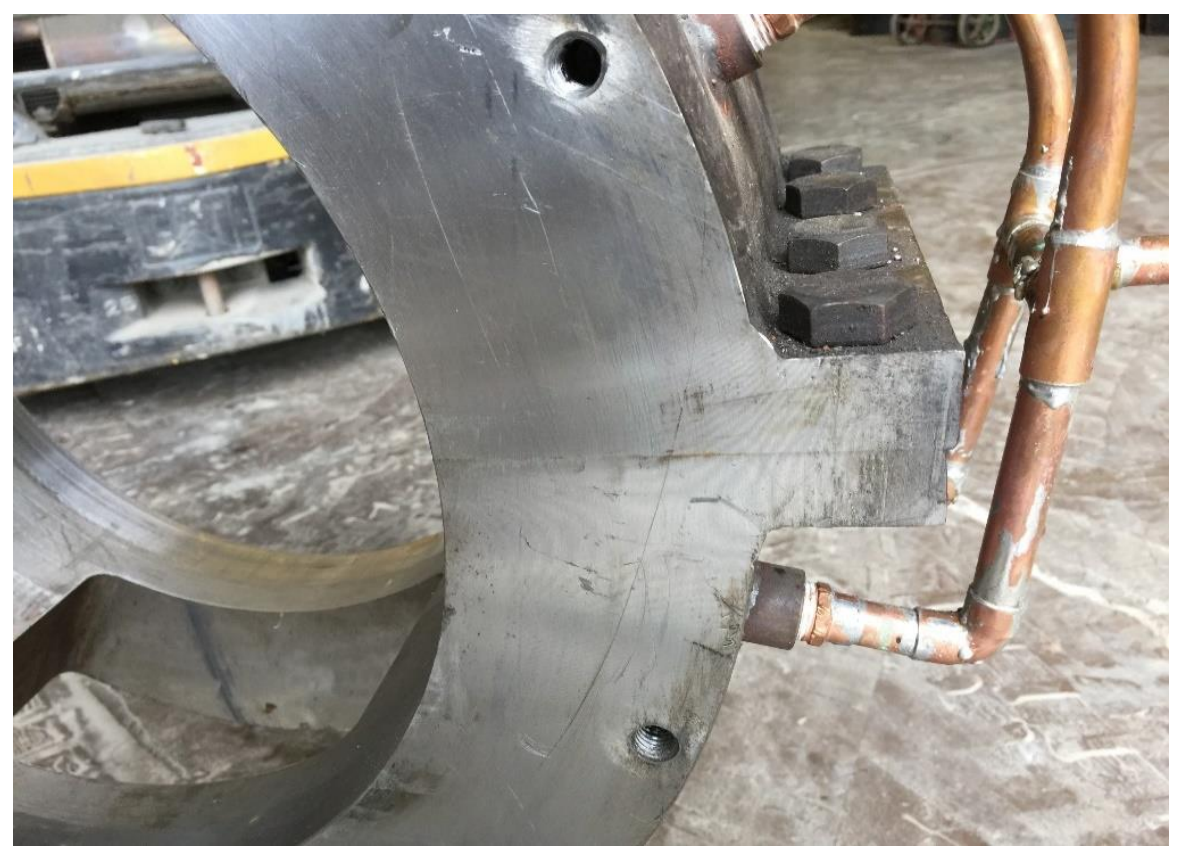

Figure 5-12: Semi Circular components bolted and welded together to form cylindrical chamber. 
The heating chamber is mounted on two parallel plates (Figure 5-13) of steel. The two parallel plates are welded on a base plate and are separated by a distance exactly equal to the width of the cylindrical chamber. The cylindrical chamber is placed in such a way that the opening for the dump door is at the bottom. The dump door arm is attached with a hinged connection (Figure 5-14) to the parallel plates so that it can be opened and closed easily. The hinged connection is placed in such a way that when the arm is closed, then the door exactly fits the opening of the heating chamber. There is a mechanism on the dump door arm to hold it in a closed portion when the raw material is being compounded. Both parallel plates have an opening exactly equal to the inner diameter of the heating chamber. These parallel plates are attached to the heating chamber with bolts and also the circular plates covering the sides of the cylindrical chamber (Figure 5-15). Both the circular plates and parallel plates have two different bolt opening arranged circularly. The inner circle of bolt openings fastens the circular plates to the parallel plates and outer circle of bolt opening fasten the heating chamber, parallel plates and circular plates together (Figure 5-15, Figure $5-10)$.

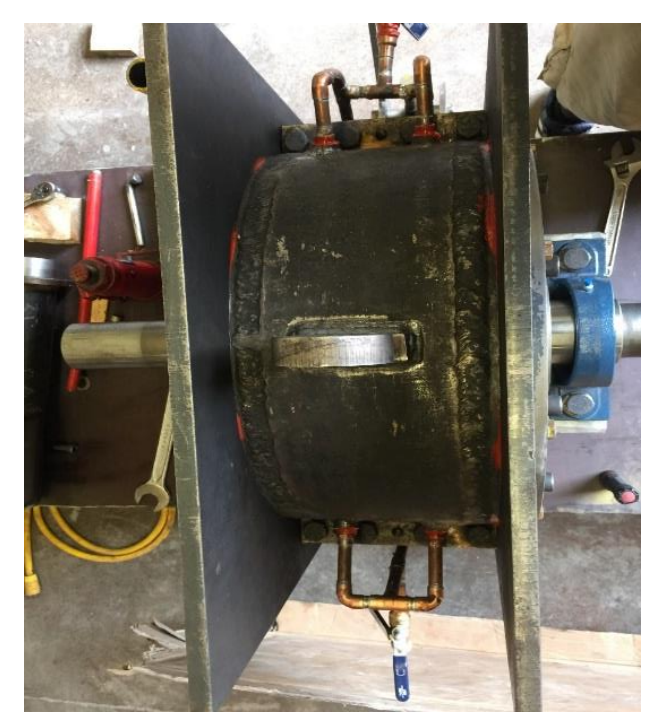

Figure 5-13: parallel plates on which the chamber is mounted. 


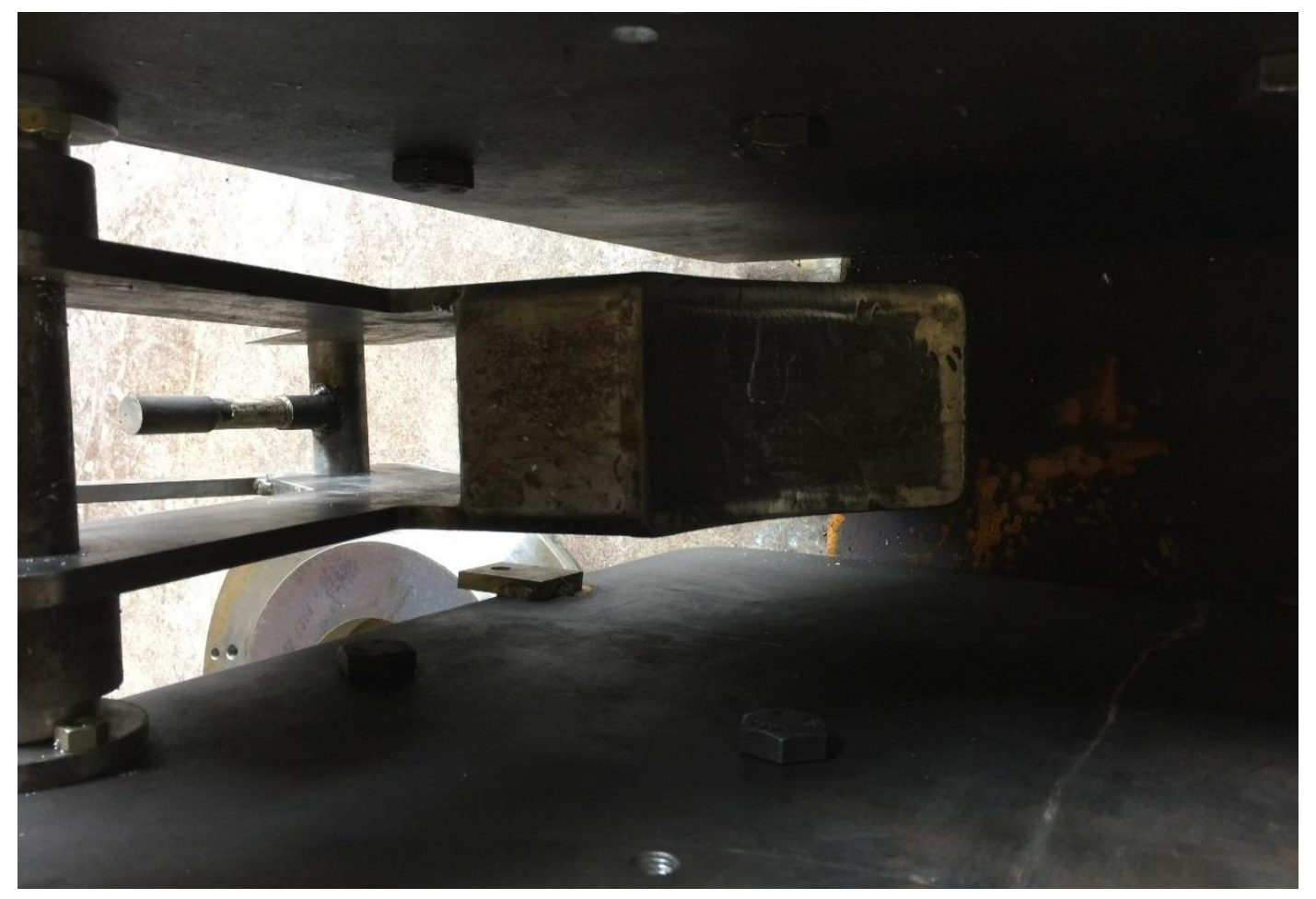

Figure 5-14: Dumping arm attached to parallel plates.

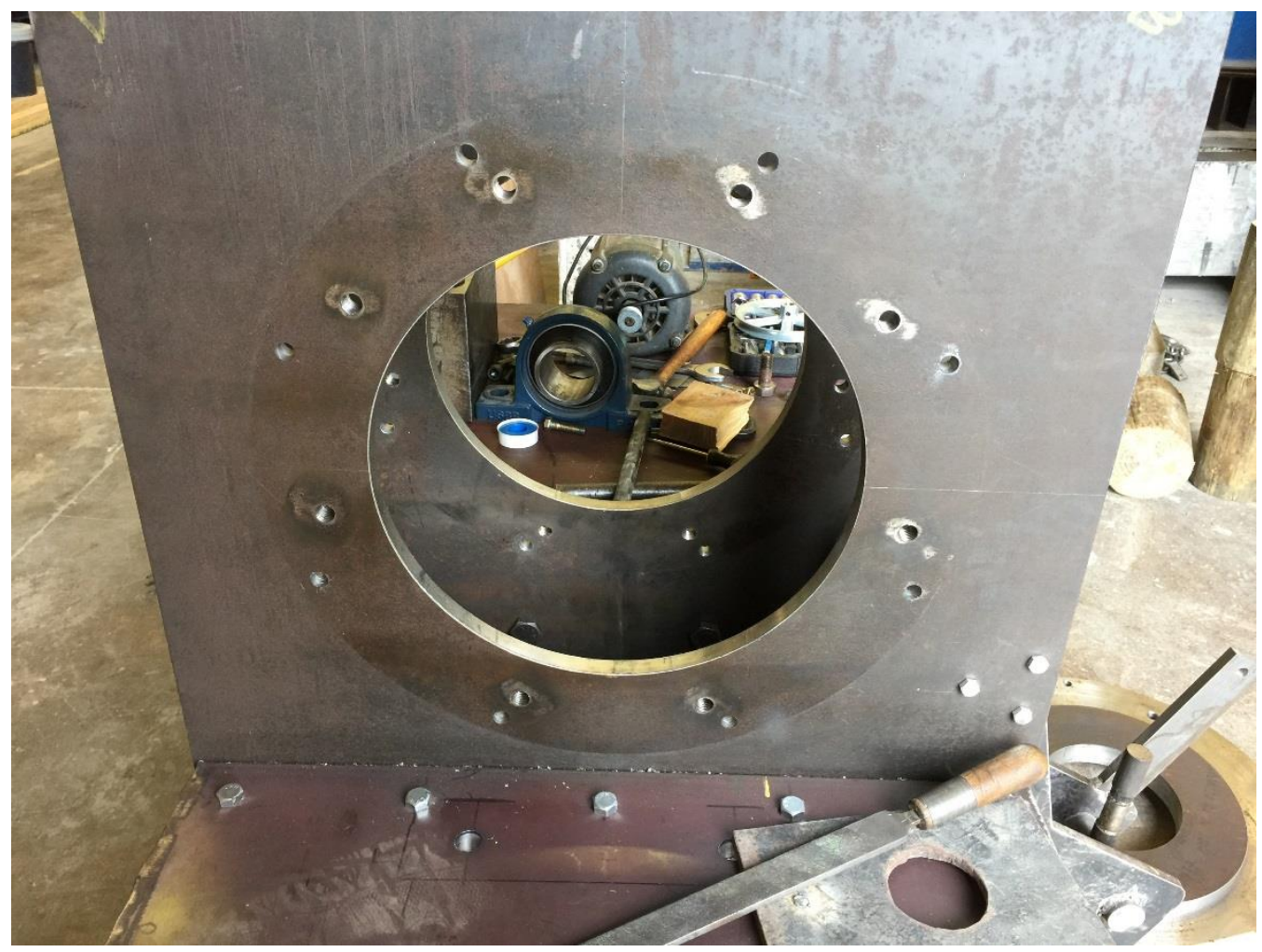

Figure 5-15: Parallel plates with provision to hold the heating chamber. 
The shaft with the grinding teeth (Figure 5-11) spins to compound the raw materials. The shaft runs all the way across the heating chamber, through the circular plates that are attached on the either side of the heating chamber. Both the circular plates have an opening with its diameter being equal to the diameter of the shaft and exactly at their center and one of the circular plate has an additional opening for the feeding tube. The portion of the shaft that is located inside the heating chamber has a larger diameter (Figure 5-16) and has attachments for the grinding blocks. There are 6 grinding blocks (Figure 5-17) that are mounted on this shaft. There are groves cut in the shaft for these blocks to be slid in and bolted (Figure 5-18), so that they can be replaced when worn out. The blocks are designed and placed in such a way that all the raw material is compounded properly. The portion of the shaft with the blocks is designed to fit exactly inside the heating chamber leaving no space between the outer surface of the blocks and inner surface of the heating chamber. Two bearings (Figure 5-19) are placed on either sides of the shaft to hold it exactly at the center of the heating chamber for smooth rotation of the shaft. The shaft is attached to a motor with the help of a coupler arrangement. One of the couplers is placed on the shaft (Figure 5-20) and the other is placed on the shaft of the motor (Figure 5-21). Both couplers fit together in their grooves. The motor drives the shaft at required rpm (3000-4000 rpm) to produce heat and compound the raw material. 


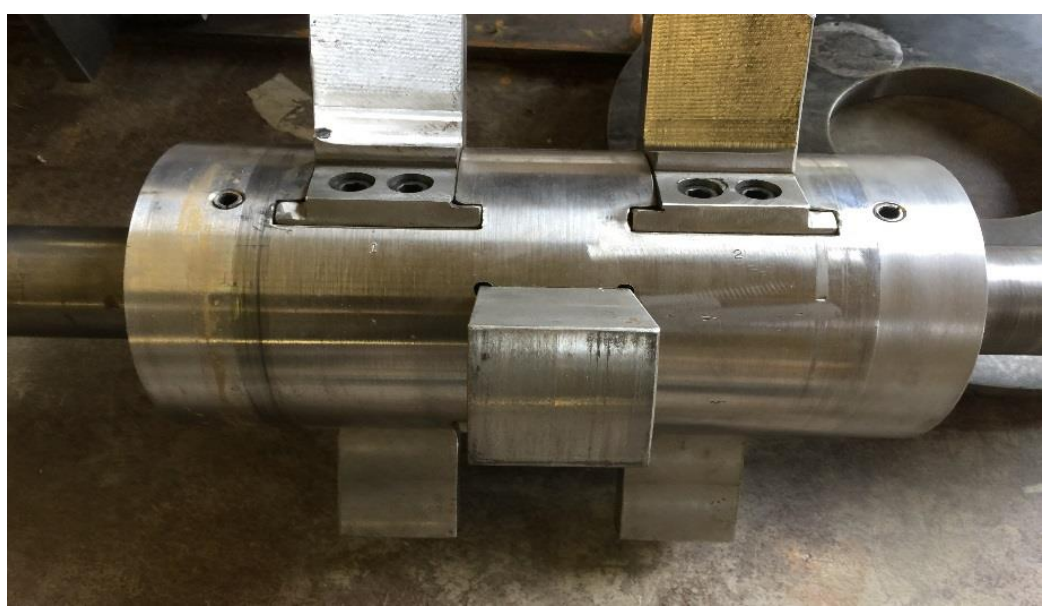

Figure 5-16: Portion of shaft that remains inside the heating chamber.

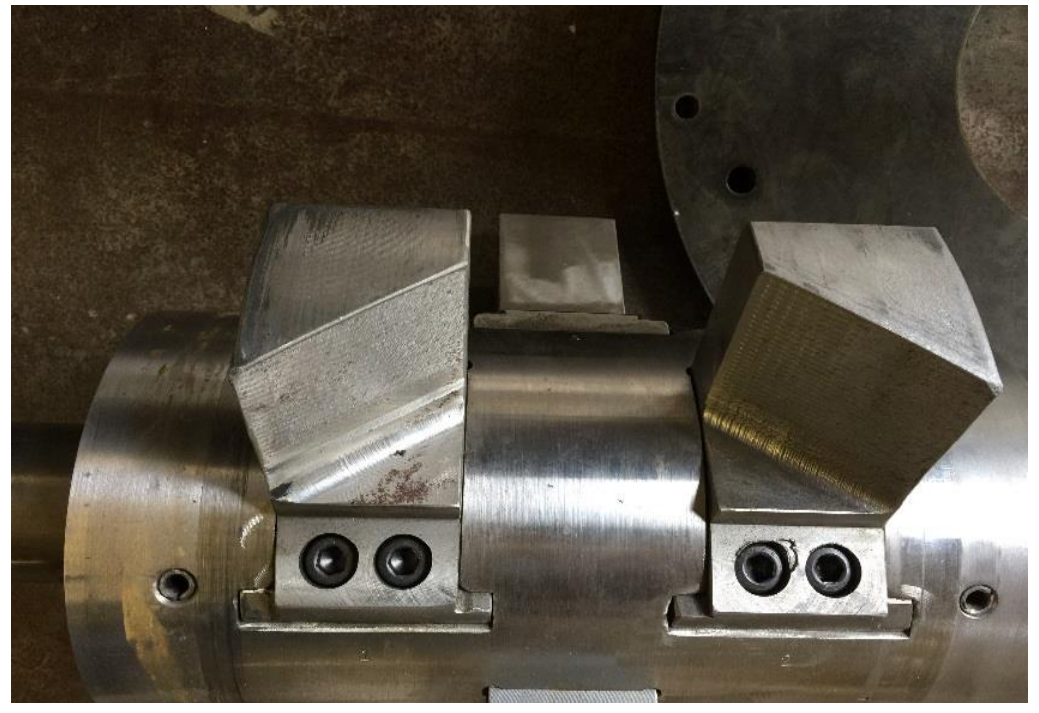

Figure 5-17: Grinding blocks mounted on shaft.

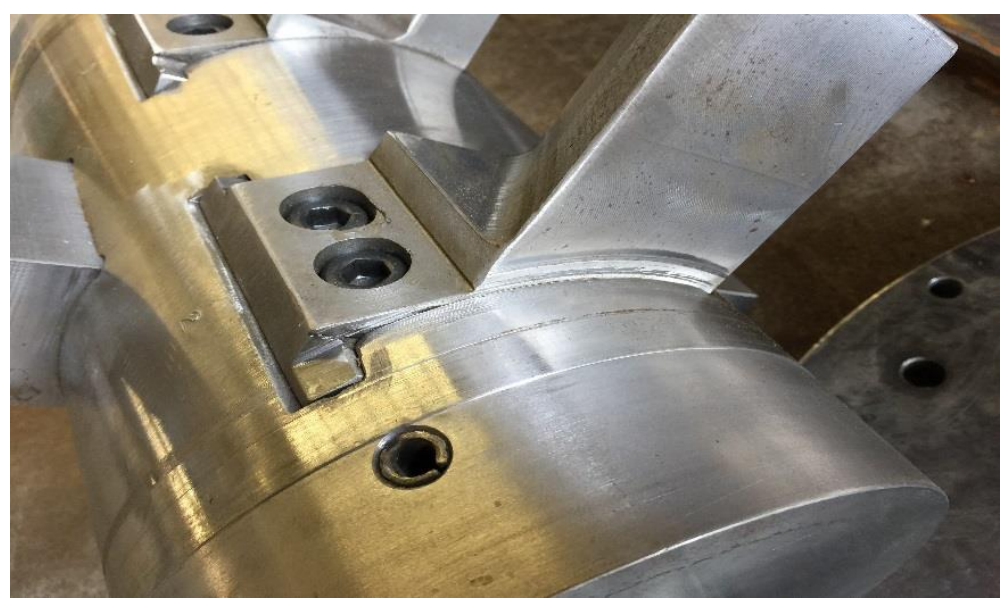

Figure 5-18: Slide in and bolt provision for the blocks. 

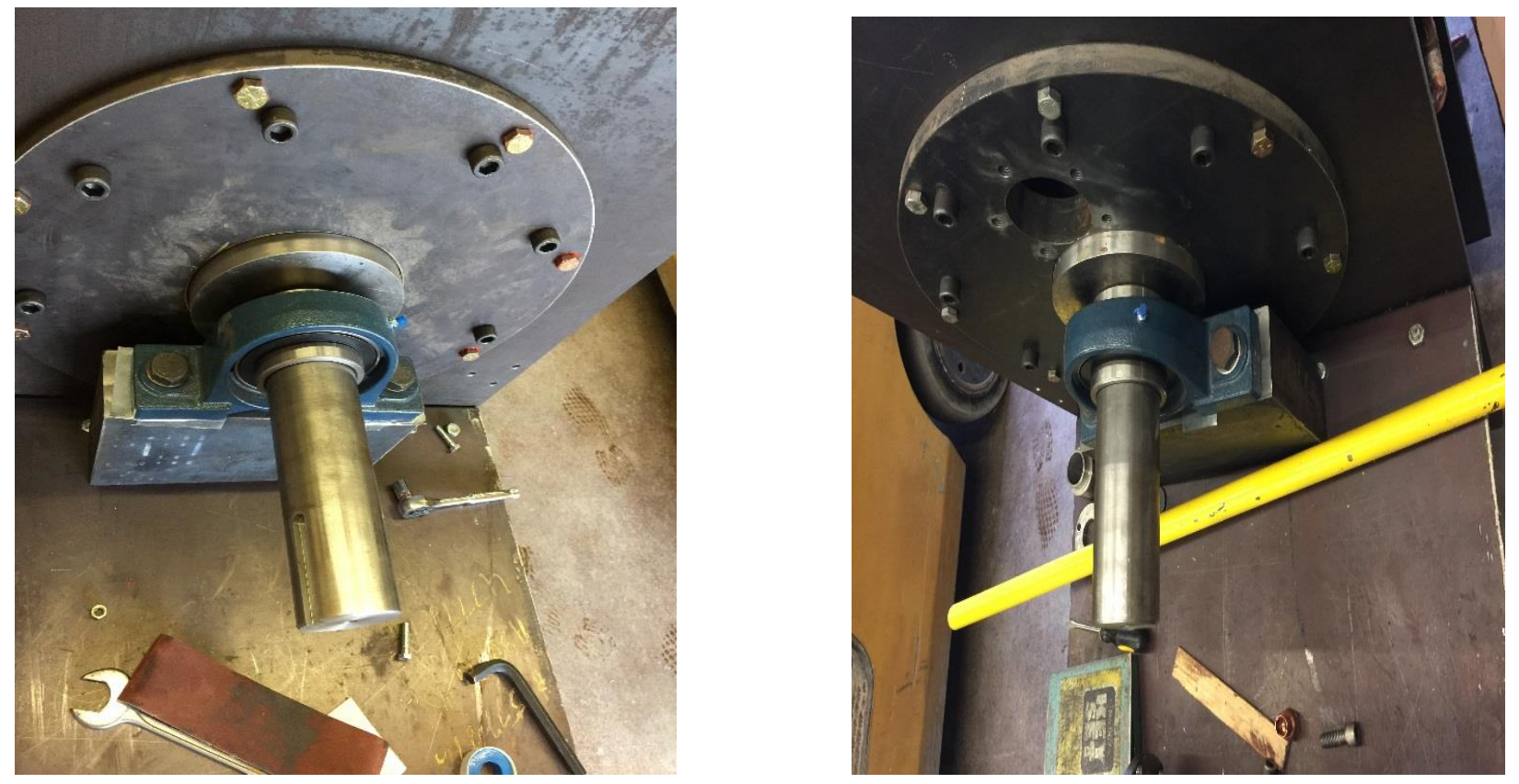

Figure 5-19: Both the bearing placed on either sides of the shaft.

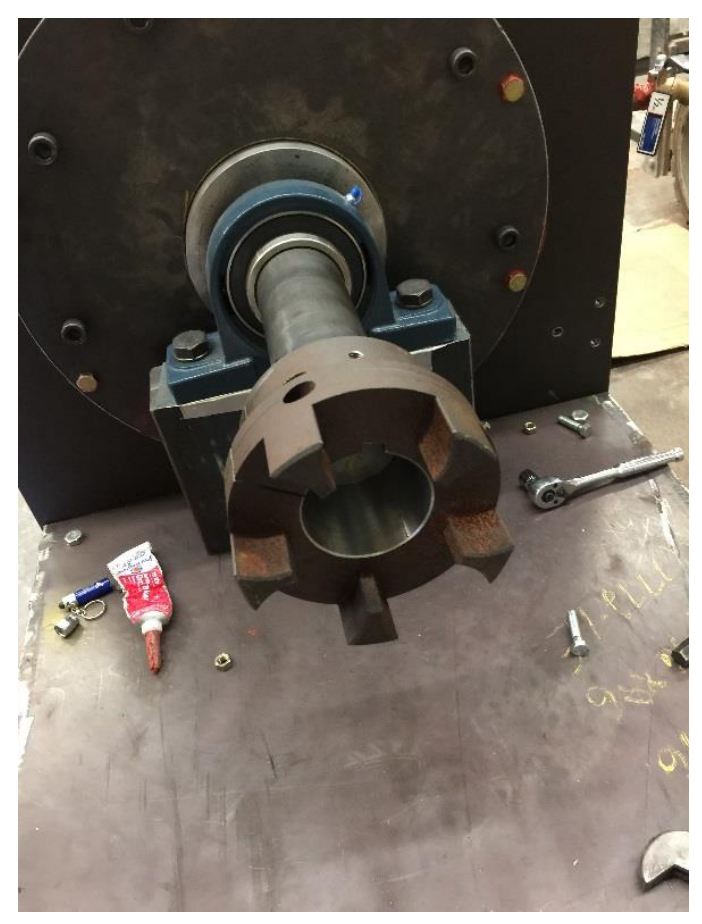

Figure 5-20: Coupler on the shaft. 


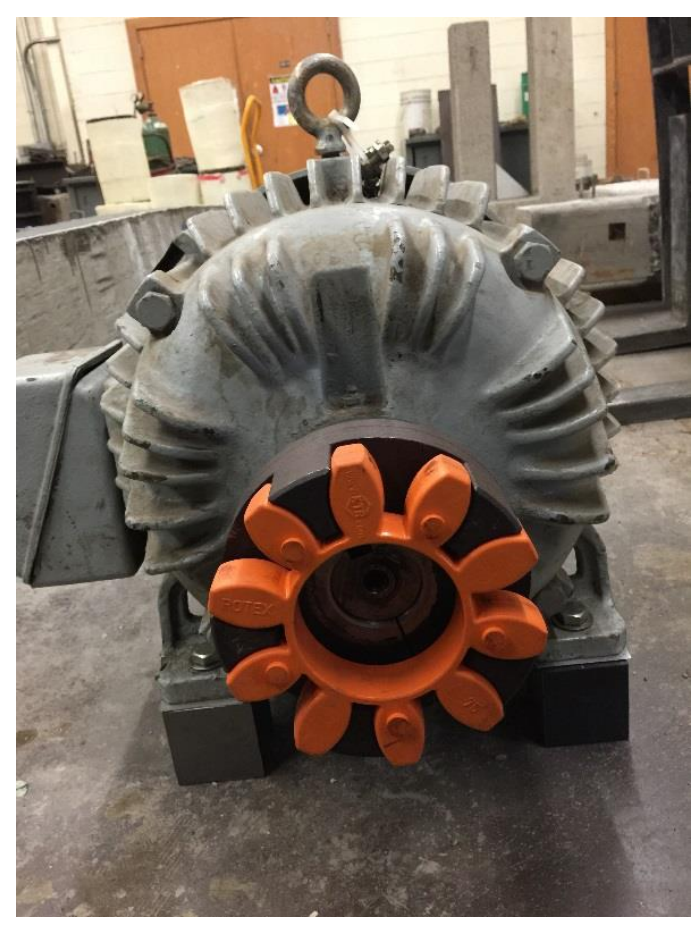

Figure 5-21: Coupler on the motor shaft.

\subsubsection{Cooling Chamber}

The cooling chamber is essential for regulating the temperature during the compounding process because the temperature in the heating chamber may rise to $300-400{ }^{0} \mathrm{C}$ due to friction of materials compounded at high speeds. The cooling chamber consists of a larger cylinder that is fitted around the heating chamber, thus creating a hollow space around the heating chamber (Figure 5-22). The cooling chamber has two inlet valves and two outlet valves through which water is circulated. The inlet and outlet valve pairs are connected (Figure 5-23) to form a single inlet and outlet source of water. The dumping arm has a similar cooling chamber and valves. The bolt holes of the cylindrical chamber to fasten it to the parallel plates are sealed with silicone which resists high temperature and doesn't shrink under thermal cycles. 


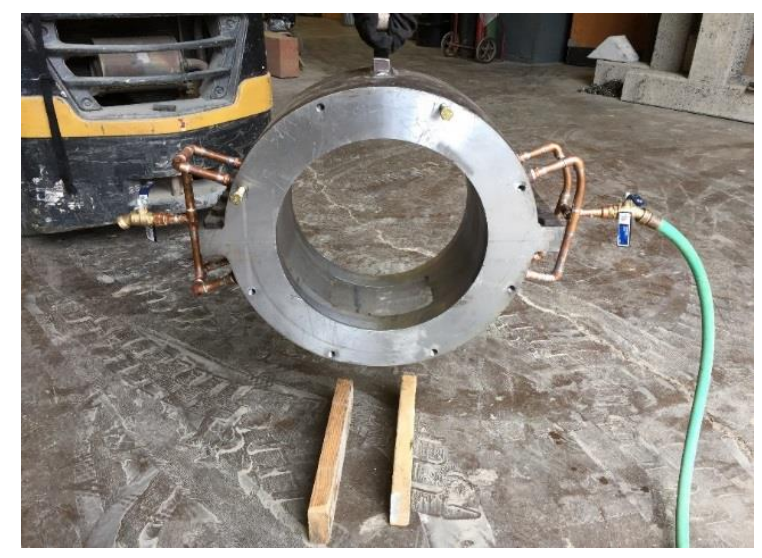

Figure 5-22: the hollow shell with cooling chamber.

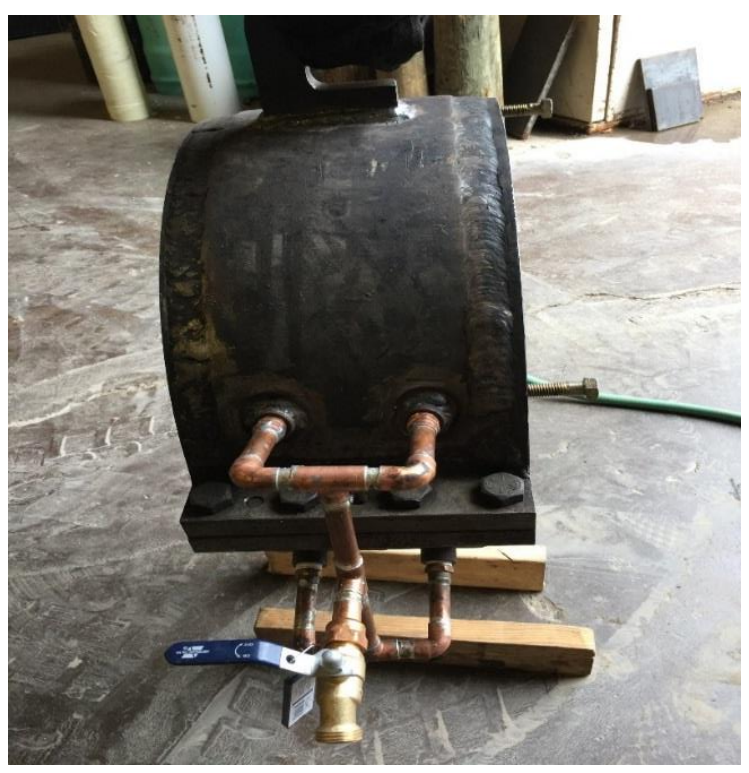

Figure 5-23: Inlet valves connected together. 


\subsection{Compounding}

\subsubsection{Introduction}

Compounding of plastics is the process in which the properties of base polymers get enhanced when compounded with additives. The comingled plastics can be compounded with various additives based on the end product requirements. The additives range from pigments, fillers, to reinforcing agents. Compounding the base polymer with additives enhances the properties like: "color, impact strength, flexibility, heat and cold resistance, UV resistance, electrical conductivity, tensile strength, lubricity ad countless other characteristics." (PolyVisions, 2016)

The base polymers used are:Comingled plastic, Plastic garbage, PP (Homo Polymer - Block Copolymer), PE, Elastomer and others.

The additives used are: pigments, glass fibers, carbon Nano particles, flame retardants, lubricants, other polymers, elastomers, nucleating agents, fillers, which are used as process aiders, Heat stabilizers, UV stabilizers, Colorants, etc.... (RTP, 2016)

- Pigments- These impart desired color to the plastic

- Lubricants- These are used to prevent plastic from sticking to the molds. (Ex: oils, waxes, soups)

- Fillers- These are used to increase elasticity and crack resistance. They also improve other properties like electrical insulation, thermal stability, strength, etc. (Ex: wood flour, mica, graphite, etc.)

- Plasticizers- They increase flexibility and toughness. (castor oil, tricresyl phosphate, etc.) 


\subsubsection{Compounding process}

The base polymer and additives are provided as shredded plastic pieces and are fed to the compounder. Shredding into small pieces ensures a better mixing and bonding of base polymers with additives. Revolving at speeds of 3000-4000 rpm, the grinding blocks and shredded raw materials rise to a temperature of about $300^{\circ} \mathrm{C}$. This heats and blends the raw materials into a homogeneous composite. Water is circulated in the cooling chamber continuously throughout the process. When the heating and bonding is completed the dump door at the bottom of the chamber is released and the compounded plastic drops out into collection tub placed below it. The collected plastic is either cooled and drawn into thin wires, placed in a compression mold to form the plastic into required shape, or used directly in a separate molding machine. The process is described in a flow chart as shown in Figure 5-24
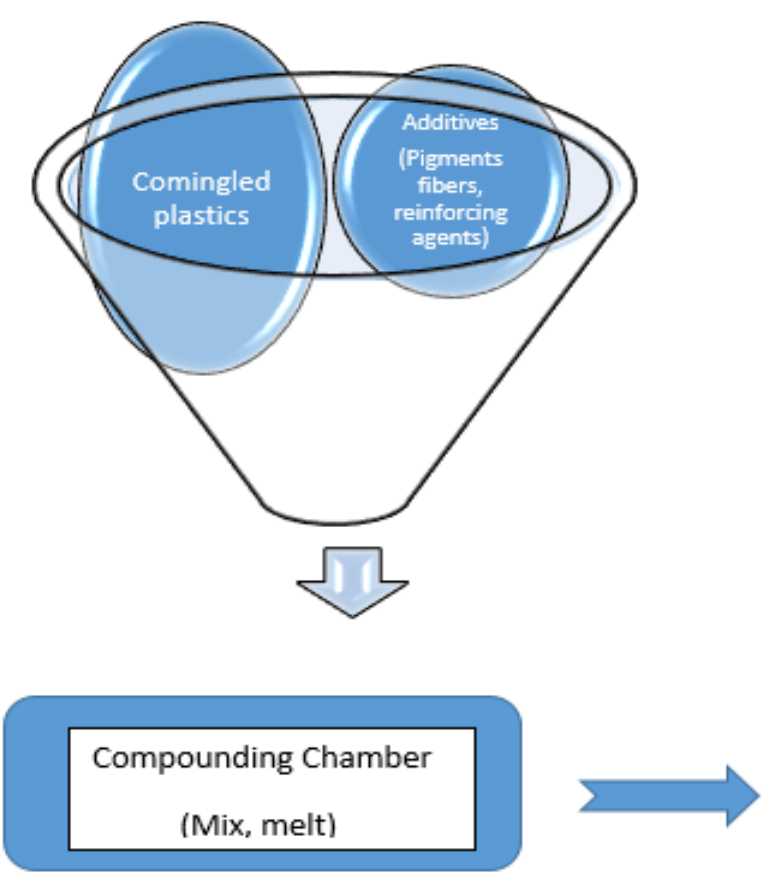

Compression, screw, injection molds, shape cool cut (pellets)

Figure 5-24: Flow Chart of the Compounding Process 


\subsubsection{Applications and Advantages}

\section{Applications:}

These compounded plastics can be widely used in many applications like:

- Particularly in construction as siding and roofing materials, replacing metal and wood. They have better durability, insulation, energy saving and weathering properties. (RTP, 2016)

- Use of compounded plastics range from small scale to large scale industries, simple toys to large furniture.

\section{Advantages of plastic compounding:}

- Compounding with additives changes the physical, thermal, electrical or aesthetic characteristics of the plastic.

- Incorporating extensive range of additives, fillers and reinforces, a wide range of properties can be achieved in conductivity, flame retardance, wear resistance, structural, and precolored. (Ramirez, 2013) 


\section{Chapter 6. Conclusions and Recommendations}

\subsection{Conclusions}

Based on the tests carried out in the Major Units Lab at West Virginia University the following conclusions can be drawn:

- The novel rehabilitation technique of underwater repair of steel piles and concrete columns with fiber reinforced polymer shell-auger system has the potential to a cost effective and safe alternative to existing rehabilitation methods

- This repair system is easier to install and more environment friendly than existing excavation techniques.

- The three designed auger attachments have been tested in lab set up and they have proven to be capable of driving the shell into the appropriate soil conditions.

- The bullet teeth auger attachment performed the best in the laboratory setup, but it also has the greatest cost.

- The application of vertical force through either vertical load or vibration is governing factor for the augering of shell with ease into the soil.

- Calculated stress values are within the material rupture limits.

- Fabricated rotary style compounder machine has potential to compound co-mingled plastics, i.e. segregation of plastics waste is not required.

- The friction between the raw material and shaft rotating at high speed (3000 rpm) generates heat for compounding. 


\subsection{Recommendations}

- Field testing of the FRP shell auger system is needed to provide a better understanding of the proposed method.

- Torque was applied manually in the lab, but mechanical application needs to be tested to ensure it is applicable to the field.

- Effect of soil interaction on the shell and the friction developed should be studied further.

- Use of different fibers as additives to compounding process to determine modifications in compounded material properties. 


\section{References}

1. ABC Diving Ltd. (2016, 08 15). Repairs and Maintenance, Cofferdams. Retrieved from ABC Diving Ltd: http://www.abcdiving.com/pages/repairmainten/cofferdams.html

2. ABC Diving® . (2016, 8 15). Dredging and Excavation. Retrieved from ABC Diving® Ltd: http://www.abcdiving.com/pages/civilworks/dredg.jetting.html

3. Air Logistics Corporation. (2012, 01 11). BP-4 Primer/Adhesive/Coating. Retrieved from Air Logistics Corporation:

http://www.airlog.com/FACS/TDS/BP\%20Primer\%204\%20Tech\%20Sheet.pdf.

4. Air Logistics Corporation. (2012, 01 11). Technical Data sheet G05 . Retrieved from Air Logistics Corporation: http://www.airlog.com/FACS/TDS/BP\%20Primer\%204\%20Tech\%20Sheet.pdf.

5. Air Logistics Corporation. (2015, 04 9). Aquawrap G-05 and G-05 Technical Datasheet. Retrieved from Air Logistics Corporation: http://www.airlog.com/FACS/TDS/Aquawrap\%20Tech\%20Sheet.pdf.

6. Air Logistics Corporation. (2015, 04 9). Highly Conformable Tape and Woven Roving Fabric Constructions. Retrieved from Air Logistics Corporation : http://www.airlog.com/FACS/TDS/Aquawrap\%20Tech\%20Sheet.pdf.

7. ArcelorMittal. (2004). Installation of Steel Sheet Piles. Retrieved from ArcelorMittal : http://sheetpiling.arcelormittal.com/uploads/files/ee6cad78a66c122d7eb19082f51d0375.pdf

8. Dam-It-Dams. (2016, 8 15). Portable Water filled Dams. Retrieved from Dam-It-Dams: http://damitdams.com 
9. Frederick T. Wallenberger, J. C. (2001). Glass Fibers. ASM international: ASM Handbook, Vol.21: composites(\#06781G). Retrieved from http://www.asminternational.org/documents/10192/1849770/06781G_p27-34.pdf

10. Gangarao, H. V., \& P.V.Vijay. (November 2010). Feasibility Review of FRP material for Strucutral Applications.

11. Houlihan, E. (April 2015). No April Fools' Day Joke: Over 61,000 U.S. Bridges need structural repair. ARTBA.

12. Kenneth Viking. (May 2002). Vibro-Driveability -A field study of Vibratory Driven Sheet Piles in Non-Cohesive Soils. Stockholm,Sweden: Doctoral Thesis submitted Dept. of Civil and Architectural Engineering, Royal Institute of Technology (KTH).

13. Lytle, C. L. (2016, April). coastal care. Retrieved from coastal care wbsite: http://plasticpollution.org/

14. Michael J. Garlich, S. P., John E. O’Leary, S. P., Katherine C. Heringhaus, T. B., \& Thomas J. Collins, S. P. (April 2010). Underwater Bridge Repair, Rehabllitation and Countermeasures. Fedral Highway Administartion.

15. Nawaguna, E. (April 2014). One in Ten U.S. Bridges in urgent need of repair. Reuters.

16. PolyVisions. $(2016,09)$. What is Thermoplastic Compounding. Retrieved from PolyVisions Inc: http://www.polyvisions.com/what-is-thermoplastic-compounding/

17. Prince Engineering. (2016, august 11). Glass Fiber Differences and Properties. Retrieved from Prince Engineering: http://www.build-on-prince.com/glass-fiber.html\#sthash.5GBvx0DC.dpbs

18. Prince Engineering-Biuild. (2016, 09 28). Glass Fiber Difference and Properties. Retrieved from Prince Engineering-Biuild on Price.com: http://www.build-on-prince.com/glass-fiber.html 
19. Ramirez, M. A. (2013, march). Polymer Science and Technology, Slide Share of Class .

20. RTP. (2016, 09). What is speciality Compounding. Retrieved from RTP Co: http://www.rtpcompany.com/about/what-is-specialty-compounding/

21. Simpsons Strong-Ti. (2016, 08 14). FX-70 Structural Repair and Protective system. Retrieved from Simpsons Strong-Ti: https://www.strongtie.com/products/rps/fx70

22. Soti, P. R. (2014). Advanced Composites for Design and Rehabilitation of Hydraulic Structures. Morgantown: Masters Thesis submitted to Dept. of CEE, CEMR, West Virginia University.

23. Statista. (2016, 09 12). Global plastic production from 1950 to 2015. Retrieved from Statista: https://www.statista.com/statistics/282732/global-production-of-plastics-since-1950/

24. US Army Corps of Engineers. (January 2013). EAST FORK BRIDGE LOAD AND RESISTANCE FACTOR RATING (LRFR) OF PILE FOUNDATION. Huntington District.

25. Zehev Tadmor, C. G. (2006). Principles of Polymer Processing. Second Edition published by A John Wiley \& Sons, Inc., Publication. 


\section{Appendix A- Stress Calculation of the Shell Wrap composite}

\section{Introduction}

The stress values are to be calculated from the strain values collected. We should take into consideration of the composite action of both FRP shell and wrap while calculating.

\section{Sample calculation}

The wrap area is converted into an equivalent shell area using modular ratio.

\section{Wrap:}

Tensile Modulus $E_{W}=2.28 \times 10^{6} \mathrm{psi}$.

Thickness ( 2 wraps) $t_{W}=2 \times 0.02875$

(Air Logistics Corporation, 2015)

$$
=0.0575 \text { inch } \text {. }
$$

\section{Shell:}

Tensile Modulus $E_{S}=1.5 \times 10^{6} \mathrm{psi}$.

Thickness $t_{S}=0.125$ inch .

Modular Ratio $n=\frac{E_{W}}{E_{S}}$

$$
\begin{aligned}
& =\frac{2.28 \times 10^{6}}{1.5 \times 10^{6}} \\
& =1.52 .
\end{aligned}
$$

Modified thickness of wrap $=n \times t_{W}$

$$
\begin{aligned}
& =1.52 \times 0.0575 \\
& =0.08763 i n c h .
\end{aligned}
$$

Total effective thickness of composite $=\left(n \times t_{W}\right)+\left(t_{S}\right)$

$$
\begin{aligned}
& =0.08763+0.125 \\
& =0.21263 \text { inch } .
\end{aligned}
$$


Calculation of stress for strain of $232 \mu$ strains at 14-inch location

Strain is same in both wrap and shell

$\varepsilon=0.000232$ (Strain gage reading)

\section{Wrap:}

$$
\begin{aligned}
& \varepsilon=\frac{\sigma_{W}}{E_{W}} \\
& \sigma_{W}=\varepsilon \times E_{W} \\
& \sigma_{W}=0.000232 \times 2.28 \times 10^{6} \\
& =530.352 \mathrm{psi} . \\
& \sigma_{W}=\frac{P_{W}}{A_{W}}
\end{aligned}
$$

Load coming on to the wrap

$$
\begin{aligned}
P_{W} & =\sigma_{W} \times A_{W} \\
& =530.352 \times 0.0575 \times 1 \\
& =30.495 \mathrm{lb}
\end{aligned}
$$

\section{Shell:}

$$
\begin{gathered}
\varepsilon=\frac{\sigma_{S}}{E_{S}} \\
\sigma_{S}=\varepsilon \times E_{S} \\
\sigma_{S}=0.000232 \times 1.5 \times 10^{6} \\
=348 \mathrm{psi} . \\
\sigma_{S}=\frac{P_{S}}{A_{S}}
\end{gathered}
$$

Load coming on to the shell

$$
\begin{aligned}
P_{S}=\sigma_{S} & \times A_{S} \\
& =348 \times 0.125 \times 1 \\
= & 43.5 \mathrm{lb}
\end{aligned}
$$

Stress in composite system $\sigma=\frac{P}{A}=\frac{43.5}{(0.21263 \times 1)}=204.58 \mathrm{ps} i$

Similarly, Stresses corresponding to other strains are calculated.

\section{Where,}

$E_{W}=$ Tensile modulus of wrap.

$E_{S}=$ Tensile modulus of shell.

$t_{S}=$ Thickness of shell.

$t_{W}=$ Thickness of wrap.

$n=$ Modulus Ratio.

$\varepsilon=$ Strain 


\section{Appendix B - Maximum Vertical load and Torque applicable on shell}

Introduction: to determine the maximum torque and vertical load that can be applied on the shell to auger it down into the soil.

Calculation: Based on the FRP shell and wrap used in the lab testing the following calculation is made. The vertical load and torque capacities vary with the materials and dimensions. This is just an example calculation

Maximum Torque applicable

$$
\begin{aligned}
& D_{o}=20.1825 \text { inch } . \\
& D_{i}=20 \text { inch. } \\
& J=\frac{\pi\left[D_{0}^{4}-D_{i}^{4}\right]}{32} \\
& J=\frac{\pi\left[(20.1825)^{4}-(20)^{4}\right]}{32} \\
& J=\frac{\pi[5920]}{32} \\
& J=580.941 \mathrm{in}^{4}
\end{aligned}
$$

Minimum failure stress (FRP)

$$
\gamma=30000 \text { psi. }
$$


Maximum applicable torque

$$
\begin{aligned}
T & =\frac{\gamma J}{\frac{D_{0}}{2}} \\
T & =\frac{[30000][580.941]}{\frac{20.1825}{2}} \\
T & =1727064.871 \mathrm{lb}-\mathrm{in} \\
& =143922 \mathrm{lb}-\mathrm{ft}
\end{aligned}
$$

This value of torque is rough estimate of maximum value that can be applied based on material properties. This calculation does take into consideration of any other factors.

\section{Maximum applicable Vertical load}

$$
\begin{aligned}
A & =\pi\left[r_{0}^{2}-r_{1}^{2}\right] \\
& =\pi\left[10.09125^{2}-10^{2}\right] \\
& =5.7566 \mathrm{in}^{2} .
\end{aligned}
$$

Minimum failure compression stress (FRP) $\sigma=25000 \mathrm{psi}$.

$$
\begin{aligned}
\sigma & =\frac{\rho}{A} \\
\rho & =25000 \times 5.7566 \mathrm{in}^{2} \\
& =143916.135 \mathrm{lb}=143.92 \mathrm{kips}
\end{aligned}
$$

Where,

$D_{o}=$ Outer Diameter.

$D_{i}=$ Inner Diameter.

$J=$ Polar moment of inertia.

$T=$ Torque.

$\rho=$ Applied load.

$\sigma=$ Compression Stress.

$A=$ Cross section Area . 
The calculated vertical load is the maximum resistance offered by the FRP shell wrap system can take. If the load is being applied using hydraulic jack against the existing structure, the capacity of the structure should also be considered. For example, let us consider the East Fork Bridge on East Lyn Lake (US Army Corps of Engineers, January 2013). The dead load distribution on the piers is calculated below:

The effective $\operatorname{span}=27 \mathrm{feet} 6$ inches.

The width of the bridge $=28$ feet.

$$
\begin{array}{ll}
\text { Thickness of slab } & =11.75 \text { inches } \\
\text { Density of concrete } & =145 \mathrm{lb} / \mathrm{ft}^{3} \\
\text { Dead weight } & =109.32 \mathrm{kips}
\end{array}
$$

The calculated dead weight is less than the maximum load capacity of the shell wrap system. So the maximum load that can be applied using a jacking system should be less than the dead weight (109.32 kips) and proper safety factor should be applied. If not, the excess (vertical) downward push might affect the stability of existing superstructure. 